\title{
Elterliche Kontroll- und Wertregulationen und die Lernmotivation des Kindes
}

I: [...] Was muss ich mir darunter vorstellen, wenn Sie sagen, es gehöre zu Ihrer Rolle, Ihrer Tochter «den Weg zu zeigen»?

H11: Ja. Einfach-. Seit sie klein war-. Während sie aufwächst, musst du [ihr] zeigen, wie man die Dinge macht und wie sie ist oder wie man das sehen muss. Und nachher-. Es wird immer anspruchsvoller im Leben, oder-. Sie muss sich [dauernd] entscheiden. [...] Ja, und wir Eltern zeigen den Weg: «Du gehst in die Schule und jetzt machst du dein Leben». Und: «K05, du musst in die Schule gehen. Weil [...] später machst du es so und so. Und es kommt darauf an, was du später werden möchtest, also musst du jetzt schon [damit] anfangen». Ja. Schauen, dass sie-. Mehr oder weniger den richtigen Weg wählt. Nicht einfach auf der Straße sitzt und die ganze Zeit nichts macht. [...] (Interview G2, $00: 10: 22)$

Motivationstheorien widmen sich der Frage, warum Menschen (und Tiere) sich so verhalten, wie sie es tun (vgl. Graham \& Weiner, 2012, S. 367; Wigfield, Muenks \& Rosenzweig, 2015, S. 9). Pintrich (2003, S. 669) meint, dass sich diesbezügliche Konzeptionen vor allem mit zwei Aspekten beschäftigen würden, nämlich damit, «what gets individuals moving (energization) and toward what activities or tasks (direction [...])». Motivationstheorien erläutern demnach, wie die Energetisierung des Individuums - das, was es in einer bestimmten Situation zum Tun und Handeln bewegt und antreibt (lat. movere) - zustande kommt, und warum es sich dabei auf bestimmte Ziele, unter mehreren möglichen, ausrichtet. Schunk et al. (2014, S. 5) definieren Motivation ferner als «the process whereby goal-directed activities are instigated and sustained» und weisen damit darauf hin, dass Motivationstheorien heute nicht nur die Genese von zielgerichteten Aktivitäten erklären, sondern auch, unter welchen Umständen diese aufrechterhalten und schließlich beendet werden. Indem es innere kognitive und affektive Prozesse beschreibt, ist 
Motivation ein genuin hypothetisches Konstrukt (vgl. Möller, 2008, S. 263). Motivationale Zustände und Prozesse müssen denn auch von außen über kognitionsund affektbezogene Aussagen von Menschen oder über die Beobachtungen der Intensität und der Persistenz des Engagements, das sie in ihren Aktivitäten an den Tag legen, erschlossen werden (vgl. Wigfield, Eccles, et al., 2015, S. 1).

Wie kommen Energetisierung und Zielausrichtung zustande? Seit Beginn des letzten Jahrhunderts suchen Motivationstheorien Antworten auf diese Frage, indem sie die Wechselbeziehung zwischen irgendwie gearteten inneren personalen Faktoren und äußeren Merkmalen der Situation zu konzipieren versuchen.

Bevor Motivationstheorien erörtert werden, die im Zuge der kognitiven Wende entstanden sind und auf erfahrungsbasierten Überzeugungen und Situationswahrnehmungen als den zentralen inneren Faktoren der Motivationsgenese abheben, stehen im folgenden Abschnitt die Konstrukte Bedürfnisse und Motive im Zentrum. Die beiden dispositionalen Konstrukte waren während Jahrzehnten die maßgeblichen intrapersonalen Größen in der (Leistungs-)Motivationsforschung und gewinnen in den letzten Jahren u. a. im Zuge eines verstärkten Interesses an affektiv-emotionalen Prozessen wieder an Bedeutung (vgl. Pintrich, 2003, S. 670; Wigfield, Eccles, et al., 2015, S. 2). Besondere Aufmerksamkeit wird hierbei John W. Atkinsons motiv-theoretischem Risiko-Wahl-Modell (1957) zuteil, welches die Grundlagen für die heutigen erwartungswert-theoretischen Ansätze der Lern- und Leistungsmotivation gelegt hat (vgl. Trautwein et al., 2012, S. 763; Wigfield et al., 2016, S. 55).

\subsection{Bedürfnisse, Motive und das Risiko-Wahlmodell von Atkinson (1957)}

Bis in die Mitte des letzten Jahrhunderts richtete sich das Interesse der etablierten Motivationsforschung auf tiefliegende, dem Bewusstsein nur begrenzt zugängliche dispositionale Merkmale von Menschen in Form von Trieben und Bedürfnissen zur Erklärung deren zielgerichteten Verhaltens:

[...] goal-directed behavior is [seen as] a joint product of the individual's internal need ([e.g.] hunger [...]) and situational incentives ([e.g.] food-related cues) that allow the expression of this need. This also means that individuals with a stronger need become more motivated by the same incentive cue than individuals with a weaker need. (Schultheiss, Strasser, Rösch, Kordik \& Graham, 2012, S. 650) 
Die jeweils aktuelle Motivation resultiert demnach aus der Aktivierung von Bedürfnissen sowie Merkmalen der wahrgenommenen Situation (vgl. Rheinberg, 2006, S. 70), wobei diese Bedürfnisse wie im vorliegenden Beispiel dranghaftprimärer Art - auf die Behebung eines physiologischen Mangels ausgerichtet - oder eher sekundärer Art - auf die Entwicklung des Selbst hin orientiert - sein können (vgl. Covington, 1992; Maslow \& Murphy, 1954; Vansteenkiste et al., 2008). Grundlegend ist in diesem Zusammenhang die Unterscheidung zwischen Approach und Avoidance: Individuen sind vom Bedürfnis geleitet, Reize, die als angenehm (appetitiv, positiv-valent) empfunden werden, aufzusuchen bzw. den Kontakt zu ihnen zu bewahren, und Reize, die als aversiv (negativ-valent) wahrgenommen werden, zu meiden (vgl. Feltman \& Elliot, 2012). An den Begriff des Bedürfnisses schließt sich derjenige des Motivs an. Von Atkinson (1957, S. 360) als «a disposition to strive for a certain kind of satisfaction, as a capacity for satisfaction in the attainment of a certain class of incentives» definiert, bezeichnen Motive stabile affektive, perzeptive und evaluative Präferenzen eines Individuums (vgl. Rothermund \& Eder, 2011, S. 91) für bestimmte situationale Anreize bzw. für spezifische Typen von Handlungssituationen (vgl. Rheinberg, 2006, S. 62). Motive sind genuin bereichsübergreifende und implizite Konstrukte, deren individuelle Charakteristika sich in der frühen Kindheit ausprägen (vgl. Haase \& Heckhausen, 2012, S. 483):

Motives are implicit in the sense that they are rooted in specialized brain systems developed over evolutionary time spans, operate outside of a person's conscious awareness and therefore have to be assessed indirectly, such as by content coding of verbal material. Such indirect motive measures predict a large array of motivational phenomena, ranging from physiological and neural responses to incentive stimuli, to economic success and political action, but frequently fail to overlap with people's explicit declarations of their motivational needs and goals. (Schultheiss et al., 2012, S. 652)

Von zentraler Bedeutung in Unterricht, Schule und Familie ist neben dem Machtund dem Anschlussmotiv (vgl. Schmalt \& Heckhausen, 2010; Sokolowski \& Heckhausen, 2010) namentlich das Leistungsmotiv: Als leistungsmotiviert gilt ein Verhalten dann, wenn sich das Individuum «einem Tüchtigkeitsmaßstab verpflichtet fühlt» (Brunstein \& Heckhausen, 2010, S. 145) und ohne direkten äußeren Anstoß Präferenzen für das Aufsuchen kompetenzrelevanter Situationen erkennen lässt $^{1}$ (vgl. Conroy, 2017, S. 26; Haase \& Heckhausen, 2012, S. 483):

\footnotetext{
${ }^{1}$ Bewertung der eigenen Leistung kann dabei an unterschiedlichen Maßstäben bzw. Bezugsnormen erfolgen (vgl. Klauer \& Leutner, 2012, S. 196-197; Rheinberg \& Fries, 2018,
} 
Eine Person mit einem stark ausgeprägten Leistungsmotiv nimmt in einer Handlungssituation eher wahr, dass man hier etwas besser oder schlechter machen kann, sie sieht viel häufiger Gelegenheiten, ihre Tüchtigkeit zu erproben und zu steigern und erlebt diese Gelegenheiten auch als anregender und wichtiger, als wenn sie stattdessen ein stark ausgeprägtes Machtmotiv besässe. [...] Das Motiv ist also so etwas wie eine spezifisch eingefärbte Brille, die ganz bestimmte Aspekte von Situationen auffällig macht und als wichtig hervorhebt [...]. (Rheinberg, 2006, S. 62-63)

Wie Atkinson (1957) in seiner für die aktuellen Theorien der Lern- und Leistungsmotivation wegweisenden erwartungs-werttheoretischen Konzeption darlegt, sind mit dem Bemessen der eigenen Tüchtigkeit die beiden Affekte der Hoffnung auf Erfolg («hope of success», S. 366) und der Angst zu scheitern («fear of failure», S. 366) verbunden, die von ihm als integrale Bestandteile des Leistungsmotivs verstanden werden, insofern sie ebenso zeitstabil wie dieses seien und interindividuell jeweils domänenübergreifend einer der beiden Affekte dominiere (vgl. Dresel \& Lämmle, 2017, S. 95). So unterscheiden sich laut Atkinson Menschen dispositional darin, in welche Richtung sie sich in Leistungssituationen ausrichten: Erfolgszuversichtliche Menschen («person[s] whose motive to achieve is stronger than [their] motive to avoid failure», S. 368) sind bestrebt, in Leistungssituationen sich dem erwünschten (appetitiven oder positiv-valenten) Zustand anzunähern, der mit Stolz über das Ergebnis («pride in accomplishment», S. 360) verbunden ist, und lassen sich bei der Bearbeitung der Aufgabe von der Hoffnung bzw. Erwartung auf Erfolg leiten:

An expectancy is a cognitive anticipation, usually aroused by cues in a situation, that performance of some act will be followed by a particular consequence. The strength

S. 56-57). Der intraindividuelle Längsschnittvergleich («Bin ich besser geworden?») wird als persönliche Bezugsnorm, der interindividuelle Querschnittsvergleich («Wo stehe ich im Vergleich zu andern?») als soziale Bezugsnorm und der lernzielbezogene Vergleich («Wo stehe ich in Relation zum angestrebten Handlungsergebnis?») als sachliche oder kriteriale Bezugsnorm bezeichnet. Zuerst orientiert an der Erzielung von einfachen Handlungseffekten, dann mit einem zunehmenden Fokus auf die Aufgabenschwierigkeit und begleitet von den Emotionen Freude und Frustration, entwickelt sich in der frühen Kindheit das Leistungsmotiv. Mit fortschreitender kognitiver Reifung und aufgrund der Reaktionen von Bezugspersonen werden zunehmend Kompetenzüberlegungen angestellt und Anspruchsniveaus bei der Aufgabenwahl definiert. Während zunächst das eigene Können im Sinne der persönlichen Bezugsnorm im Zentrum steht, wird das eigene Handeln spätestens mit Eintritt in die Schule auch am sozialen Maßstab bemessen - was sich in den Emotionen Stolz und Scham manifestiert (vgl. Haase \& Heckhausen, 2012, S. 484; Holodynski \& Oerter, 2008, S. 550-553; Pekrun \& Perry, 2014, S. 121). Während für die Entwicklung des Leistungsmotivs von Kindern Belohnungen und Anerkennung wichtig sind, spielen sie für das ausgebildete - als Disposition konzipierte Leistungsmotiv von Erwachsenen keine Rolle mehr (vgl. Rothermund \& Eder, 2011, S. 105). 
of an expectancy can be represented as the subjective probability of the consequence, given the act. (Atkinson, 1957, S. 360)

Misserfolgsängstliche («person[s] who [are] more strongly disposed to be fearful of failure», S. 369) zeigen in Leistungssituationen eine dispositionale Ausrichtung darauf, den unerwünschten (negativ-valenten) Zustand des Sich-Schämens zu vermeiden, der sich bei einem allfälligen Misserfolg einstellt («shame and humiliation as a consequence of failure», S. 360) und lassen sich bei der Bearbeitung von einer Aufgabe von der damit verbundenen Angst bzw. Erwartung des Scheiterns leiten. Atkinson zufolge ist die in einer Leistungssituation erlebte Motivation das Produkt aus dem Grad der Erfolgserwartung (subjektive Wahrscheinlichkeit, die Aufgabe bewältigen zu können) und dem Grad des subjektiven Erfolgsanreizes, die beide eine gewisse minimale Ausprägung aufweisen müssen. In seinem Modell der Risiko-Wahl postuliert Atkinson nun, dass Menschen bei einer freien Aufgabenwahl eigentlich diejenigen wählen müssten, die ein aus ihrer Sicht mittleres Anspruchsniveau aufwiesen. Anforderungen, die subjektiv als schwierig oder als einfach eingeschätzt würden, motivieren das Individuum nämlich daher nicht zum Handeln, weil im ersteren Fall die Erfolgswahrscheinlichkeit und in letzterem Fall der Anreiz zu gering sei. Er argumentiert, dass Aufgaben, die subjektiv als anforderungsreich, aber nicht als zu schwierig eingeschätzt werden («tasks of intermediate difficulty», S. 363), angesichts der umgekehrten Proportionalität zwischen Anreizwert und Erfolgserwartung eigentlich mit dem maximalen Erfolgsversprechen verbunden seien (vgl. Atkinson, 1957, S. 367). Atkinson betont nun allerdings, dass dies nur für erfolgszuversichtliche Individuen gelte. Misserfolgsängstliche würden die angemessenen Aufgaben mittleren Anforderungsniveaus dahingegen in der Regel meiden und solche wählen, die sie subjektiv als einfach oder aber als schwierig taxierten. Da die Bearbeitung mittelschwieriger, eigentlich sinnvoller Aufgaben die eigene Tüchtigkeit am klarsten transparent macht, wirkten sie für die Betroffenen am bedrohlichsten. Mit der Wahl von einfachen oder von sehr schweren, bis zu einem gewissen Grad spekulativen Aufgaben ließe sich dahingegen die befürchtete Konfrontation mit der eigenen Inkompetenz verhindern, indem sich nämlich im ersteren Fall ein sicherer Erfolg einstelle und im letzteren Fall Rückschlüsse auf die eigene Tüchtigkeit nicht nahelägen (vgl. Atkinson, 1957, S. 366). Wie Brunstein und Heckhausen (2010, S. 163-183) in ihrer detaillierten Zusammenfassung der Befundlage zu Atkinsons Postulaten darlegen, bestätigt sich zwar das abweichende Wahl- und Leistungsverhalten von Misserfolgsängstlichen, doch konnte das von ihm vermutete Motivationsmuster (maximale Motivation bei Aufgaben tiefen und hohen Anforderungsgrades sowie minimale Motivation bei mittlerem Anforderungsgrad) 
nie zweifelsfrei bestätigt werden. Das Modell als Ganzes fand bezüglich der Aufgabenwahl und der Ausdauer bei der Aufgabenbearbeitung gute Bestätigung. Die Leistungsmenge dürfte demnach von der Motivationsstärke abhängig sein, bezüglich der Leistungsqualität scheint dies allerdings nur sehr bedingt zu gelten, was sich damit erklären lässt, dass hier - wie Atkinson in späteren Arbeiten selber zeigte - neben der Motivation auch das Fach- und Methodenwissen des Individuums eine entscheidende Rolle spielt (vgl. Brunstein \& Heckhausen, 2010, S. 181-183). Als grundsätzliche Einschränkungen der Aussagekraft des Modells haben sich aber insbesondere die zwei Aspekte erwiesen, die damit zusammenhängen, dass es sich dabei um eine Theorie handelt, die auf dem Motivkonzept beruht. In einer Leistungssituation ist demnach allein das (implizite) Motiv, die persönliche Tüchtigkeit zu beweisen, maßgeblich - nach dem Motto «je schwieriger, desto attraktiver» (Heckhausen \& Heckhausen, 2010, S. 454). Andere Anreize (z. B. Lob, Anerkennung, Tadel), die mit einem Leistungsergebnis verbunden sein können, spielen in diesem Ansatz keine Rolle. Entscheidend sind die Affekte Stolz und Scham (vgl. Rothermund \& Eder, 2011, S. 109-110). Wie sich empirisch gezeigt hat, besteht die von Atkinson postulierte negative Korrelation zwischen Erwartung und Wert nur bei diesem spezifischen affektiven Anreiz (Stolz) (vgl. Dresel \& Lämmle, 2017, S. 97-98). Beim Vorliegen anderer subjektiver Aufgabenanreize (z. B. situationales Interesse bzw. intrinsischer Wert oder instrumenteller Aufgabenwert) (vgl. Abschnitt 5.5.1) zeigt sich dahingegen konsistent ein positiver Zusammenhang zwischen der Erwartungs- und der WertKomponente (z. B. Battle, E. S., 1966; Eccles \& Wigfield, 1995; Feather, 1988; Trautwein et al., 2012). Ein zweites Problem ergibt sich aus der Bereichsunabhängigkeit des Konstrukts Leistungsmotiv, auf das sich Atkinsons Theorie stützt. Wie Dresel und Lämmle (2017, S. 96) anmerken, erweisen sich Instrumente, welche die Leistungsmotivation (auch) bereichsspezifisch erheben, regelmäßig als prädiktiver für das tatsächliche Verhalten als solche, die (lediglich) domänenunspezifische diesbezügliche Daten erfassen (vgl. Elbe, Wenhold \& Müller, 2015). Dies verweist allgemein darauf, dass die Effekte, die von Bedürfnissen und Motiven auf das leistungsbezogene Handeln ausgehen, über subjektive, auf die Situation bezogene Gedankengänge der Lernenden vermittelt werden (vgl. Pintrich, 2003, S. 670).

\subsection{Persönliche Ziele}

Mit der kognitiven Wende in der Psychologie ist der «trait approach to motivation» (Graham \& Weiner, 2012, S. 370) und der damit verbundene Fokus auf 
weitgehend unbewusste, domänenunspezifische, stabil den Menschen antreibende Bedürfnisse und Motive als Hauptuntersuchungseinheiten (vgl. Pintrich, 2003, S. 680-681) denn auch abgelöst worden von der sozial-kognitiven Sichtweise (vgl. Bandura, 1986), die das Subjekt in Wechselwirkung mit seiner spezifischen sozialen und kulturellen Umwelt in den Blick nimmt und die Kontextund Erfahrungsgebundenheit seines Wahrnehmens, Interpretierens und Handelns betont. Diese psychologische Akteurstheorie (vgl. Abschnitt 2.1) portraitiert den Menschen als «self-organizing, proactive, self-regulating, and self-reflecting» (Bandura, 2006, S. 164). In Theorie und Forschung zur Lern- und Leistungsmotivation stehen seither explizite, für Bewusstsein und Sprache zugängliche persönliche Ziele $^{2}$ sowie selbst- und aufgabenbezogene Überzeugungen und Situationseinschätzungen im Zentrum, die zur Erklärung von Ausdauer, Engagement und Erfolg beim Kompetenzerwerb in Schule, Unterricht, Elternhaus und Freizeit herangezogen werden (vgl. Wigfield, Eccles, et al., 2015, S. 2).

Schultheiss et al. (2012, S. 653) definieren persönliche Ziele als «subjectively meaningful representations of anticipated end-states delineating what a person wants to achieve, maintain, or avoid in his or her current life situation». Solche Repräsentationen von erwünschten Handlungsfolgen können durch Eltern, Lehrkräfte und andere Bezugspersonen von außen ans Individuum herangetragen, gemeinsam mit anderen in partizipativen Situationen entwickelt (vgl. Abschnitt 2.2.2.3) oder von ihm selber generiert werden (vgl. Locke \& Latham, 2006, S. 265), wobei sich besonders in ersterem Fall die Frage stellt, unter welchen Bedingungen diese fremdinitiierten Ziele internalisiert und handlungswirksam werden (vgl. Abschnitt 5.6.2).

Als subjektiv repräsentierte Soll-Werte sind Ziele laut Kleinbeck (2010, S. 285) die eigentlichen «Dreh- und Angelpunkte» der Handlungsregulation, insofern als sie das Individuum in der prädezisionalen Phase (vgl. Rubikon-Modell; Achtziger \& Gollwitzer, 2010, S. 311) zu Handlungen anregen und es in der präaktionalen Phase in Richtung des angestrebten Ergebnisses planen und und die nötigen Mittel organisieren lassen. Ebenso dienen sie in den aktionalen und postaktionalen Phasen als Orientierungshilfe, indem sie in Relation zu handlungsbezogenem Feedback (vgl. Abschnitt 5.4) laufend zur Bewertung des bisher Erreichten bzw. zur Erfolgs-Misserfolgseinschätzung herangezogen werden:

Eine Diskrepanz [zwischen Ergebnissen und Zielen] fordert zur Ursachenforschung heraus und stellt die Weichen für das weitere Vorgehen. Entweder kommt es zu einem

\footnotetext{
${ }^{2}$ Ziele höherer Hierachiestufen werden mitunter auch als «explizite Motive» bezeichnet (vgl. Haase \& Heckhausen, 2012, S. 484).
} 
erneuten Zielerreichungsversuch mit verstärktem Einsatz persönlicher Leistungsvoraussetzungen (Steigerung von Anstrengung und Ausdauer) bzw. veränderten Handlungsstrategien oder zur Senkung der Zielhöhe bis hin zur Zielaufgabe. (Kleinbeck, 2010, S. 287)

Ziele können danach unterschieden werden, ob sie das Handlungsergebnis betreffen oder sich auf den Prozess beziehen (Kleinbeck, 2010, S. 289). Ergebnis- bzw. Leistungsziele sind Endpunkte von Handlungen (z. B. das Ziel, «den Übertritt in die Abteilung A zu schaffen»). Sind sie erreicht, können diese aufgegeben werden. Die Handlungsrelevanz eines Prozess- bzw. Lernziels bleibt dahingegen längerfristig erhalten, insofern als dieses auf die stetige Kompetenzerweiterung gerichtet ist (z. B. «das Beste geben, zu dem man fähig ist») (vgl. Abschnitt 2.2).

Ziele werden sodann als hierarchisch organisierte Konstrukte des Selbstkonzepts eines Individuums verstanden. Handlungsziele - bei der Aufgabe, eine schriftliche Hausarbeit zu verfassen, könnten diese beispielsweise die Menge, die inhaltliche Qualität, die sprachliche Qualität und die Termintreue betreffen - lassen sich einerseits weiter in konkrete Teilziele unterteilen, andererseits zu übergeordneten Zielklassen im Sinne von persönlichen Standards und Zielorientierungen (vgl. Abschnitt 4.2.1.2) im Umgang mit Leistung, Wissenserwerb und Zusammenarbeit zusammenführen (vgl. Kleinbeck, 2010, S. 288).

Zuoberst in der Zielhierarchie stehen schließlich allgemeine menschliche Werte wie Verantwortungsbewusstsein, Wahrheitstreue etc. (vgl. Rokeachs Konzeption terminaler Werte, Abschnitt 5.5.1.2), die als «guiding principles for the lives of individuals (personal values) and social groups or society as a whole (social values)» dienen (Schultheiss et al., 2012, S. 653). Solcherlei gesellschaftlich geformte und sozial vermittelte Normen (vgl. Abschnitt 2.1), aber auch individuelle Ausprägungen von Zielorientierungen bzw. Bezugsnormen sowie Bedürfnisse und Motive, verleihen einzelnen Handlungszielen anderen gegenüber Gewicht bzw. Priorität. Handelnde müssen also in der Phase der Entscheidungsfindung, aber stetig auch während der aktionalen Phase, eine Wahl darüber treffen, welche möglichen Ziele sie vorziehen und gegenüber welchen sie sich abschirmen möchten. Nebst weitgehend automatisierten Prozessen geringer Bewusstheit umfasst dies auch Problemlöseprozesse, in denen allein oder mit Hilfe anderer darüber räsoniert wird, warum dem einen oder anderen Ziel einen höheren Wert beigemessen werden sollte (vgl. Abschnitt 5.6).

So sind Menschen im Alltag durch komplexe situative Anforderungen sowie durch ihre Vorlieben ständig damit konfrontiert, mehrere Handlungsziele gleichzeitig verfolgen zu müssen bzw. verfolgen zu wollen. Gelingt dies in einer 
Situation nicht - a) weil die gleichen kognitiven Verarbeitungsmodi bei der Aktivität involviert sind (z. B. die Sprachproduktion beim Führen eines Telefonats und dem gleichzeitigen Schreiben), b) weil mit der Verfolgung eines höher gelagerten Ziels andere ebenso stark gewichtete außer Reichweite gelangen (z. B. die Ziele, eine Hausarbeit den eigenen Ansprüchen gerecht zu verfassen und intensiv am Leben der eigenen Familie zu partizipieren) oder c) weil die eigenen Ziele nicht kompatibel mit denjenigen von Bezugspersonen sind (z. B. das Ziel des Kindes am Sonntagabend Hausaufgabenhilfe von den Eltern zu bekommen und deren Ziel, einen ruhigen Filmabend zu genießen) -, so liegt ein Zielkonflikt vor, der laut Kleinbeck (2010, S. 299) durch die angesprochene Höherbewertung eines der beiden Handlungsziele (mittels des Abwägens der Bedeutsamkeit auf höheren Ebenen der Zielhierarchie, vgl. Abschnitt 5.5.1.4), durch die serielle Bearbeitung der beiden Ziele oder durch Kompromisse (um beide Ziele gemeinsam zu verfolgen) gelöst werden kann.

Zentral ist dabei, dass das Individuum auch die zur Verfügung stehenden Mittel (means) in den Blick nimmt, mit denen die Ziele (ends) erreichbar scheinen. Meist führen verschiedene Teilhandlungen bzw. Aktivitäten zum gleichen Ziel auf einer höheren Hierarchieebene, wodurch immer auch diese Aktivitäten sowie die dabei beteiligten Personen, Verfahren und Gegenstände hinsichtlich ihrer Bedeutsamkeit («Wie angenehm?», «Wie interessant?», «Wie wichtig?», «Wie nützlich?», «Wie unangenehm oder nachteilig?») für die Erreichung des Ziels eingeschätzt werden (vgl. Higgins, 2007). Die in Abschnitt 5.5.1 näher zu erläuternde Theorie des Subjective Task Value von Jacquelynne S. Eccles und Kolleg*innen (vgl. Eccles, 2005; Wigfield \& Eccles, 1992) bezieht sich auf diesen Umstand, insofern als sie verschiedene Funktionalitäten beschreibt, die Lernende in Aktivitäten bzw. Aufgaben erkennen, und erklärt, wie diese den Wunsch des Individuums beeinflussen, sich den Aufgaben zu widmen und sie zu bearbeiten (vgl. Wigfield et al., 2016, S. 57). Es sind demnach bestimmte Merkmale der Aufgaben - nebst dem Handlungsziel und seinen Folgen insbesondere die zur Zielerreichung erforderlichen Teilhandlungen sowie die dabei beteiligten Personen und Gegenstände -, die einer (situativen) Bedeutsamkeits- bzw. Werteinschätzung unterzogen werden, indem sie mit (generelleren) subjektiven Wert-Überzeugungen abgeglichen werden (analog zum in Abschnitt 4.2 erläuterten Zusammenspiel zwischen situativen und generelleren Überzeugungen bei der elterlichen Handlungsentscheidung).

Motivieren bedeutet im Kontext des unterrichtlichen und häuslichen Lernens und Unterstützens folglich, die sozialen, inhaltlichen, methodischen und personalen Aufgabenmerkmale und Ziele (laufend) so zu gestalten, dass das Kind vor, während und nach der Bearbeitung eine möglichst aktivierende Funktionalität in ihnen erkennt (vgl. auch Abschnitt 2.2.2.3). Oft wird das Kind allerdings «den 
Sinn» eines Ziels «nicht sehen», aus der Sicht der Lehrkräfte und/oder der Eltern zielführende Mittel und Strategien außer Acht lassen oder ablehnen sowie ein Leistungsergebnis nicht angemessen gewichten. Die Erziehenden greifen in solchen Situationen zu verbalen Wertregulationen und versuchen das Kind mithilfe von Argumenten, die getragen sind von ihren eigenen wertbezogenen Überzeugungen (vgl. Abbildung 4.1), von der Bedeutsamkeit des Ziels bzw. der Aufgabe zu überzeugen. In den Abschnitten 5.6 bis 5.6.2 wird diese Form der elterlichen Unterstützung näher erläutert.

\subsection{Subjektive Kontrolleinschätzungen}

In Einklang mit den Annahmen der Erwartungs-Wert-Konzeption Atkinsons stellt sich dem Individuum bei der Zielsetzung und der Zielverfolgung allerdings nicht nur die Frage nach dem Wollen, sondern immer auch nach dem Können. Einerseits unterscheidet sich je nach Situation die Distanz zwischen dem wahrgenommenen Ist-Zustand und dem erwünschten Soll-Zustand - nicht jedes Ziel ist gleich hoch gesteckt und kann mit gleicher Sicherheit erreicht werden (endsexpectations) -, andererseits stellt sich die Frage, inwiefern einem die nötigen Mittel (means-expectations) zur Verfügung stehen, die es zur erfolgreichen Überbrückung der Distanz (means-ends-expectations) braucht (vgl. Bargh, Gollwitzer \& Oettingen, 2010, S. 268).

Günstige Kontrollkognitionen - erfahrungsabhängige generalisierte Überzeugungen sowie situative Einschätzungen zum eigenen Einfluss über Situationen, Handlungen und Handlungsergebnisse (vgl. Flammer, 2015, S. 504; Perry, Chipperfield \& Stewart, 2010, S. 43; Preiser \& Sann, 2010, S. 349) - haben sich unter Labor- und Feldbedingungen in einer Vielzahl von entscheidungs-, motivationsund emotionspsychologischen Studien als starke Prädiktoren für das psychische und körperliche Wohlbefinden von Individuen bzw. für das Ausmaß und die Qualität ihres Handelns erwiesen:

[...] across the life span, from earliest infancy to oldest age, individual differences in perceived control are related to a variety of positive outcomes, including health, achievement, optimism, persistance, motivation, coping self-esteem, personal adjustment, and success and failure in a variety of life domains. (Skinner, E. A., 1996, S. 549)

Mit Blick auf die Lern- und Leistungsentwicklung von Kindern und Jugendlichen bilanzieren Wigfield, Eccles, et al. (2015) die Befundlage zu - wie sie es nennen - «competence-related beliefs» (S. 3) mit den Worten: 
In general, when children answer [the] question [ 'can I do the task'] affirmatively, they try harder, persist longer, perform better, and are motivated to select more challenging tasks. (Wigfield, Eccles, et al., 2015, S. 3)

Auch Perry, Hladkyj, Pekrun und Pelletier (2001), die über zwei Semester den Einfluss wahrgenommener persönlicher Kontrolle (z. B. «I have a great deal of control over my academic performance in my psychology course», «The more effort I put into my courses, the better I do in them») auf eine breite Palette kognitiver, motivational-affektiver und behavioraler Konstrukte bei 524 Hochschulstudierenden untersucht haben, konstatieren positive Zusammenhänge mit einer Vielzahl derselben:

High-academic-control students exerted more effort, reported less boredom and anxiety, were more motivated, used self-monitoring strategies more often, felt more in control of their course assignments and of life in general, believed they performed better, and obtained higher final grades. (Perry et al., 2001, S. 776)

Mit Blick auf die Kausalitäten und die Stärke der Zusammenhänge fassen Linnenbrink-Garcia und Patall (2016) die Befundlage sodann wie folgt zusammen:

Expectancies for success most strongly predict performance, even when previous performance is controlled, and generally precede and predict students' values [...], though possibly less so for females [...]. (Linnenbrink-Garcia \& Patall, 2016, S. 92)

So klar die überragende Bedeutung von subjektiven kompetenz- bzw. erfolgsbezogenen Einschätzungen und Überzeugungen für die Erklärung des Lernengagements, der dabei erlebten Emotionen und des erzielten Ergebnisses zutage tritt, so deutlich wird in den zitierten Stellen auch, wie vielgestaltig die Erwartungsbzw. Kontrollkomponente über die Jahre geworden ist. Noch stärker als die Wert-Komponente (vgl. Abschnitt 5.5.1) ist sie aus einer ganzen Reihe unterschiedlicher theoretischer Perspektiven bearbeitet worden (z. B. Perry et al., 2010; Skinner, E. A., 1996; Usher, 2016). Dabei sind einzelne Dimensionen des Konstrukts zwar kontextualisiert und inhaltlich ausdifferenziert, aber oft mit ähnlichen oder den bereits bestehenden Termini versehen worden. Ferner sind umgekehrt inhaltlich weitgehend deckungsgleiche Begriffe lediglich terminologisch neu gefasst worden (vgl. Marsh et al., 2012, S. 432; Murphy \& Alexander, 
2000, S. 5), so dass, wie Usher (2016) meint, eine Situation entstanden sei, die sie als «downright confusing» (S. 146) bezeichnet ${ }^{3}$.

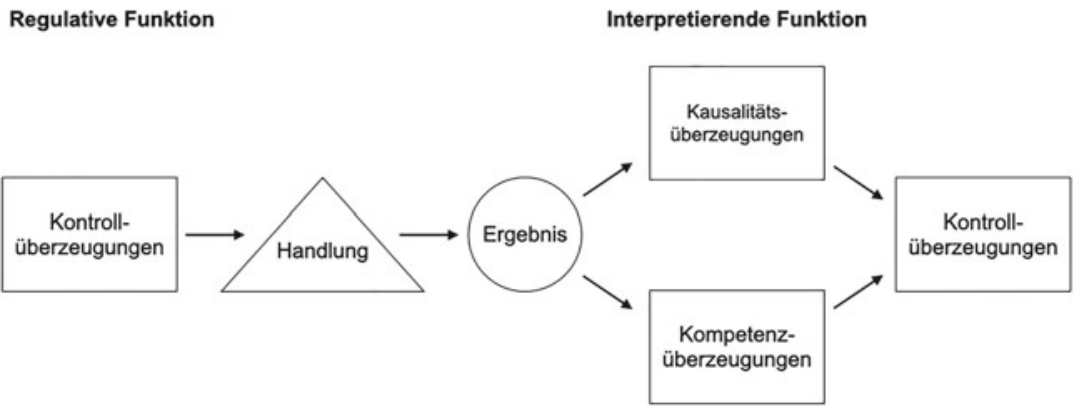

Abbildung 5.1 Schematische Darstellung des Kompetenzsystems (Adaptiert nach Skinner, E. A., 1995, S. 20; mit freundlicher Genehmigung von (C) SAGE Collage 2020. All Rights Reserved)

Mit der Absicht, die verschiedenen Kontroll-Konstrukte zu ordnen und auf der Basis der Prämisse, wonach Menschen ein angeborenes Bedürfnis haben, sich als kompetente und wirksame Akteure in ihrer sozialen und physischen Umwelt wahrzunehmen, die erwünschte Zustände hervorbringen können und unerwünschte zu vermeiden vermögen, versteht die Arbeitsgruppe um Ellen A. Skinner (Skinner, E. A., 1995, 1996; Skinner, E. A., Chapman \& Baltes, 1988) die verschiedenen Kontroll-Konstrukte als Facetten eines kompetenzbezogenen Überzeugungssystems (competence system), welches die Aufgabe hat, das Handeln eines Individuums mit Blick auf die jeweiligen Ziele zu evaluieren und zu regulieren:

\footnotetext{
${ }^{3}$ Skinner (1996, S. 549) macht zwei Gruppen von kontrollbezogenen Konstrukten aus: In der ersten Gruppe taucht der Term control in deren Bezeichnung auf. Dazu gehören z. B. «personal control, sense of control, locus of control, cognitive control, agenda control, vicarious control, illusory control, outcome control, primary control, secondary control, action control, decisional control, predictive control, informational control, and proxy control». In der zweiten Gruppe wird der Term control zwar nicht für die Benennung des Konstrukts verwendet, inhaltlich scheinen aber große Überschneidungen mit den Konstrukten der ersten Gruppe zu bestehen. $\mathrm{Zu}$ diesen Konstrukten gehören u. a. «helplessness, efficacy, agency, capacity, mastery, effectance, effectiveness, autonomy, self-determination, competence, contingency, causal attributions, explanatory style, responsibility, blame, probability of success, and outcome expectancy».
} 
Hence, all theories of perceived control are attempts to map the competence system. These theories have in common that they try to explain how control experiences contribute to the construction of beliefs, and how these beliefs in turn promote or undermine effective interactions. The theories differ in the specific part of that process on which they focus. (Skinner, E. A., 1995, S. 20)

Wie die schematisierte Darstellung einer Handlungssequenz illustriert (vgl. Abbildung 5.1), kommen Kontroll-Kognitionen im Kompetenzsystem im Rahmen zielorientierter Interaktionen mit der Umwelt zweierlei Funktionen zu: a) vor oder während des Handelns dienen sie der Regulation der Intensität und Qualität dieses Handelns und b) nach dem konkreten Handeln - bzw. in Handlungspausen, wenn auf das bisher Erreichte zurückgeblickt wird - werden sie zur Interpretation und Evaluation der (Teil-)Ergebnisse herangezogen. Die dabei erzielten Konklusionen zum Einfluss interner und externer Faktoren gehen wiederum in die Kontrollüberzeugungen ein und werden über diese vermittelt handlungswirksam (vgl. Abschnitt 5.3.3.1). Die Sequenz wird stetig durchlaufen und «funktioniert [...] wie ein sich selbst verstärkendes System» (Köller \& Möller, 2010, S. 772). Im Folgenden werden die drei in Abbildung 5.1 genannten Gruppen von Überzeugungen (Kontroll-, Kausalitäts- und Kompetenzüberzeugungen) im Kontext von Lern- und Leistungssituationen näher beleuchtet. Mit Blick auf die im Rahmen der vorliegenden Studie durchzuführende Analyse von elterlichen evaluativen Feedbacks ist es unerlässlich, dass die drei Kategorien von kontrollbezogenen Überzeugungen und ihre Subkategorien inhaltlich klar voneinander abgegrenzt werden, aber ebenso deutlich zutage tritt, in welcher Relation sie zueinander stehen. Dies wird in den folgenden Abschnitten mit Hilfe des in Abbildung 5.2 dargestellten Handlungs-Kontroll-theoretische Modell von Skinner et al. (1988) vorgenommen.

\subsubsection{Kontrollüberzeugungen}

Kontrollüberzeugungen bzw. situationsspezifische Kontrolleinschätzungen (control beliefs / control appraisals) (vgl. Abbildung 5.1) drücken das Ausmaß der Sicherheit aus, mit der Schülerinnen und Schüler vor oder während ihres Lernhandelns glauben, das Lernziel bzw. bestimmte Klassen von Lernzielen erreichen zu können (vgl. Köller \& Möller, 2010, S. 771). Wie das in Abbildung 5.2 dargestellte Handlungs-Kontroll-theoretische Modell von Skinner, E. A. et al. (1988) verdeutlicht, steht bei solchen Kognitionen der Bezug zwischen dem/der Handelnden und der Aufgabe bzw. dem Ergebnis im Vordergrund: «Wie sehr habe 




Abbildung 5.2 Handlungs-Kontroll-theoretisches Modell (Adaptiert nach Skinner, E. A. et al., 1988, S. 118; mit freundlicher Genehmigung von (C) American Psychological Association 2020. All Rights Reserved)

ich die Aufgabe im Griff?». In der Fachliteratur existiert eine Reihe von Begriffen, die im Kern auf den subjektiv wahrgenommenen Einfluss des Handelnden auf das Handlungsergebnis, auf die «agent-ends relations» fokussieren: u. a. sense of control, subjective control, perceived control, control expectancy (vgl. Skinner, E. A., 1995, S. 553). In der Erwartungs-Wert-Theorie von Eccles und Kolleg*innen erfasst das Konstrukt expectancy for success («How well do you expect to do in reading/English next year?») (Durik et al., 2006) diese Dimension und in der Kontroll-Werttheorie der Lern- und Leistungsemotionen von Pekrun die sog. subjective control (vgl. Pekrun, 2006, S. 318). Wird die Wahrscheinlichkeit als hoch eingeschätzt, eine Aufgabe bewältigen bzw. den erwünschten Zielzustand erreichen zu können, so liegt beim Individuum eine zuversichtliche Erwartung bzw. Emotionen wie Hoffnung und (Vor-)Freude (vgl. Pekrun, 2006, S. 320) vor. Wird die Wahrscheinlichkeit der erfolgreichen Handlungsdurchführung hingegen als gering eingeschätzt, herrscht die Emotion Hoffnungslosigkeit vor sowie ein Verhalten das von Rückzug, Verhinderung, Passivität und Apathie im Sinne des learned-helplessness-Konzepts gekennzeichnet ist (vgl. Peterson, Maier \& Seligman, 1993; Seligman, 1975). Kontrollüberzeugungen sind laut Skinner (1995, S. 32) zwar die «primary regulatory beliefs» im Kompetenzsystem eines Menschen, allerdings schwingen dabei immer mehr oder weniger 
deutlich Überzeugungen zur Effektivität verschiedener Mittel zur Zielerreichung (Kausalitätsüberzeugungen) sowie zur persönlichen Verfügbarkeit solcher Mittel (Kompetenzüberzeugungen) mit: z. B. «Ich habe das Ziel bzw. die Aufgabe im Griff, weil ich die zielführenden Strategien kenne und einzusetzen weiß».

\subsubsection{Kompetenzüberzeugungen}

Kompetenzüberzeugungen (vgl. Abbildung 5.2) sind Kognitionen eines Individuums darüber, inwiefern ihm persönlich die notwendigen kognitiven, motivationalvolitionalen und sozialen Ressourcen zur Verfügung stehen, um seine Wünsche und Ziele als Handlungen realisieren zu können (Flammer, 2015, S. 504). Gedankliche Prozesse, welche die «agent-means relations»(Skinner, E. A., 1996, S. 553) fokussieren, evaluieren mit anderen Worten das Vorhandensein eigenen Wissens aber auch eigener Tüchtigkeit, um die Anforderungen, die eine Aufgabe oder Situation stellt, zu bewältigen. Im Englischen wird hierzu zwischen ability und capability unterschieden:

Though small, a distinction exists between the meaning of 'ability' and 'capability'. Ability, which comes from the Latin habilis, refers to a skill or competence in doing or performing that has already been acquired. Capability refers to the potential to develop a skill or competency in the future or to perform a given task under varying conditions. I judge myself as able to write; however, I may not judge myself as capable of writing a novel this summer. In other words, capability depends in part on one's ability but also on other cognitive and motivational variables, such as effort and persistence, and on one's assessment of contextual demands. [...]. A person's beliefs therefore reflect both actuality and potentiality [...]. (Usher, 2016, S. 147, Hervorhebungen im Original)

Usher macht hier auf zwei Dimensionen des Selbst (vgl. Harter, 2006) aufmerksam, die von zwei unterschiedlichen Theoriefamilien mit zwei unterschiedlichen Konstrukten bearbeitet werden: Fragen nach dem Handlungspotential (capability) stehen beim Konstrukt der Selbstwirksamkeit (self-efficacy expectations), von Albert Bandura (1986, 1997) im Rahmen der sozialkognitiven Theorie entwickelt, im Vordergrund. Fragen nach der actuality, der Gegebenheit von Kompetenzen in bestimmten Domänen (ability), in denen gehandelt werden soll, verweisen auf das Fähigkeitsselbstbild (ability self-concept), das in der Selbstkonzept-Theorie (vgl. Shavelson, Hubner \& Stanton, 1976) im Zentrum steht. 


\subsubsection{Selbstwirksamkeit: «Wie sicher bin ich mir, dass ich es machen kann?»}

Definiert als «people's beliefs about their capabilities to produce designated levels of performance that exercise influence over events that affect their lives» (Bandura, 1994, S. 71) bezeichnen Selbstwirksamkeitserwartungen das subjektive Vertrauen, mit den eigenen fachlichen und überfachlichen Kompetenzen selbstreguliert spezifische Aufgaben bzw. bestimmte Aufgabenklassen zielführend bearbeiten zu können (vgl. Bong \& Skaalvik, 2003, S. 5; Gaskill \& Woolfolk Hoy, 2002, S. 186; Wigfield, Eccles, et al., 2015, S. 7), auch wenn diese als herausfordernd, mehrdeutig und schlecht-strukturiert erscheinen ${ }^{4}$. Das Konstrukt verweist somit auf gedankliche «can do statements» (Usher, 2016, S. 148) des Individuums, welches sich dabei in erster Linie an einem in vergangenen Handlungserfahrungen gebildeten persönlichen «goal mastery standard» (Zimmerman, Schunk \& Di Benedetto, 2017, S. 317) orientiert: z. B. «Ich bin sicher, dass ich den ganzen Abend eisern arbeiten kann, auch wenn andere mich zum Fernsehen einladen», «Wenn ein Problem auftaucht, kann ich es aus eigener Kraft meistern» (Schwarzer \& Jerusalem, 2002, S. 39-40). Wie sich im leistungsthematischen Bereich mittlerweile in hunderten von Studien gezeigt hat, erweisen sich Einschätzungen eigener Selbstwirksamkeit unabhängig vom Alter, der Schulform, dem Unterrichtsfach und dem Fähigkeitsniveau als starker Prädiktor für die Initiierung von Lernhandlungen, für die Wahl des Schwierigkeitsgrads von Aufgaben, für den Grad der Anstrengung bei der Aufgabenbearbeitung, für die Handlungspersistenz angesichts von Hindernissen und zwischenzeitlichen Misserfolgen und ebenso für Leistungsergebnisse, wo sich die Zusammenhänge im Bereich zwischen $\mathrm{r}=.50$ und $\mathrm{r}=.70$ bewegen (vgl. die Übersichtsdarstellungen bei Klassen \& Usher, 2010; Schunk \& Di Benedetto, 2015; Schunk \& Pajares, 2009). Dem Zusammenspiel der Variablen Selbstwirksamkeit, Zielorientierungen, Lernstrategien und Leistungsergebnisse wurde u. a. in einer Studie von Liem, Lau und Nie (2008) in Singapur bei 1475 Neuntklässlern im Fach Englisch nachgegangen. Theoriekonform erwies sich die Selbstwirksamkeitserwartung bei Kontrolle des Vorwissens, vermittelt über die Zielorientierung und selbstregulative Prozesse, als substanzieller Prädiktor des Leistungszuwachses: Hohe Selbstwirksamkeit war mit günstigeren Zielorientierungen (d. h. erhöhte

\footnotetext{
${ }^{4}$ Pekrun (2006) zieht es vor, hierbei von Handlungskontrolle (action control) zu sprechen: Der Ausdruck «self-efficacy expectancy» sei missverständlich, insofern als er entgegen der inhaltlichen Ausgestaltung des Konstrukts durch Bandura «the overall agency of an individual, including his or her efficacy to produce outcomes» signalisiere, statt einfach «appraisals of being able to produce an action» (S. 318).
} 
Lernzielorientierung bzw. stärkere Annäherungs-Leistungszielorientierung) assoziiert. Diese wiederum gingen mit der Wahl von förderlichen Lernstrategien (deep learning) einher, welche sodann direkt mit einem Leistungszuwachs in einem Englischtest verbunden waren. Wie dies Abbildung 5.1 andeutet, stehen Selbstwirksamkeitseinschätzungen und Leistungsergebnisse in bidirektionaler Beziehung: Erfolge und Bewältigungserfahrungen sind nicht nur eine Folge von erhöhten Selbstwirksamkeitseinschätzungen, sondern als «enactive mastery experience» (Bandura, 1997, S. 80), vermittelt über Interpretationsprozesse (vgl. Abschnitt 5.3.3.1), gleichzeitig auch die bei Weitem wichtigsten Informationsquellen des Individuums zur Ermittlung der eigenen Fähigkeit, Ziele selbstgesteuert zu erreichen. Aber auch die Beobachtung erfolgreicher Problemlöseprozesse anderer, die das Individuum als ähnlich kompetent einschätzt wie sich selbst (vicarious experience), die Überzeugungsversuche und evaluativen Feedbacks anderer («Ich bin sicher, dass du das kannst») (social persuasion, vgl. Abschnitt 5.7) sowie die eignen physiologisch-affektiven Reaktionen - z. B. erhöhte Herzfrequenz und Angst vor Leistungsereignissen (physiological and affective states) - erweisen sich wie von Bandura (1997, S. 79-115) postuliert, je als einzelnes oder in Kombination als Prädiktoren für Selbstwirksamkeitseinschätzungen (vgl. Chen \& Usher, 2013).

\subsubsection{Fachliches Fähigkeitsselbstkonzept: «Wie gut bin ich auf diesem Gebiet?»}

Das Konstrukt Fähigkeitsselbstkonzept (self-concept of ability, academic selfconcept) umfasst Einschätzungen und Überzeugungen eines Individuums hinsichtlich seiner akademischen Kompetenzen. Gedanklich beschäftigt sich dieses dabei mit der Höhe, der Differenziertheit oder der Variabilität ${ }^{5}$ seiner schulischen Fähigkeiten: «Ich bin ein sehr begabter Schüler», «Mathematik bereitet mir keine Schwierigkeiten, aber für Sprachen besitze ich einfach keine Begabung» bzw. «Für Physik fehlt mir die Begabung, daran wird sich auch nichts mehr ändern» (Stiensmeier-Pelster \& Schöne, 2008, S. 63). Ausgehend von der Definition von Shavelson et al. (1976) wird das Fähigkeitsselbstkonzept (FSK) als hierarchisches und multidimensionales Konstrukt verstanden (vgl. Marsh \& Craven, 2006; Marsh et al., 2012): So können sich die Aussagen über eigene Fähigkeiten wie im ersten Beispiel in globaler Art auf das Gesamt schulischer Anforderungen oder aber spezifischer auf Domänen in unterschiedlichem Differenzierungsgrad beziehen (Bereichsspezifisches FSK: sprachlich-philosophisch

\footnotetext{
${ }^{5}$ Vgl. hierzu die Ausführungen zu impliziten Fähigkeits- oder Intelligenztheorien in Abschnitt 4.2.
} 
vs. mathematisch-naturwissenschaftlich / Fächerspezifisches FSK: z. B. Englisch vs. Geschichte / Inhaltsspezifisches FSK: z. B. Grammatik vs. Literaturanalyse / Aufgabenspezifisches FSK: z. B. Vokabellernen vs. Textbearbeitung etc.) (Stiensmeier-Pelster \& Schöne, 2008, S. 63). Das globale akademische Selbstkonzept bildet wiederum zusammen mit den Selbstkonzepten, die mit Bezug $\mathrm{zu}$ anderen Lebenssphären gebildet wurden (u. a. social self-concept, physical self-concept), das generelle Selbstkonzept eines Individuums. Shavelson et al. (1976) postulierten, dass je höher in der Hierarchie die einzelnen Facetten der Selbstkonzepte angesiedelt seien, desto stabiler und desto bedeutsamer diese für den Selbstwert (self-esteem, self-worth) seien, «the overall evaluation of one's worth or value as a person» (Harter, 2006, S. 509) (vgl. Abschnitt 4.1.2 und Abschnitt 5.5.1.4). In der empirischen Forschung zum Fähigkeitsselbstkonzept der letzten dreißig Jahre (zsf. Marsh et al., 2012) steht vor allem die Höhe der von den Lernenden wahrgenommenen Fähigkeiten im Zentrum. Dabei zeigt sich eine ausgeprägte Domänen- und Aufgabenspezifität: Obwohl Leistungen in Mathematik und Sprache hoch korreliert sind, erweisen sich das mathematische Selbstkonzept und das sprachliche Selbstkonzept konsistent als zwei kaum korrelierte, distinkte Facetten, was die postulierte streng hierarchische Ordnung des akademischen Selbstkonzepts zumindest für die höheren Strukturebenen in Frage stellt (vgl. Möller \& Trautwein, 2015, S. 184). Gleichzeitig erweisen sich das mathematische und sprachliche Selbstkonzept als substanzielle Prädiktoren für Leistungsmaße auf beiden Gebieten, wobei die Stärke des Zusammenhangs in dem Maß, wie diese fach- und aufgabenspezifisch erhoben werden, zunimmt (vgl. Marsh \& Craven, 2006; Marsh, Hau, Artelt, Baumert \& Peschar, 2009; Valentine, DuBois \& Cooper, 2004). Das Internal/External-Frame-of-Reference-Modell (I/E-Modell) von Marsh (1986b) erklärt diese z. T. paradoxen Befunde damit, dass die Schülerinnen und Schüler ihre Fachleistungen einerseits im sozialen Vergleich beurteilen (sich also an einem externalen Bezugsrahmen orientieren) und daraus ihr Bild der eigenen Fähigkeiten in den spezifischen Fächern entwickeln («Wie gut bin ich in Mathematik?»). Andererseits nutzen sie aber auch einen internalen Bezugsrahmen und stellen dimensionale Vergleiche an: «Wie gut bin ich in Mathematik im Vergleich zu Deutsch?». Wie die hohe Korrelation zwischen sprachlichen und mathematischen Leistungen vermuten lässt, kontrastieren die Schülerinnen und Schüler dabei ihre Leistungen in beiden Domänen offenbar über Gebühr: Nach der Logik «The better I am at mathematics, the poorer I am at verbal subjects relative to my good math skills» (Marsh et al., 2012, S. 439) werden Stärken und Schwächen in der einen oder der anderen Domäne gedanklich in übertriebener Weise betont (selbst wenn die Schülerinnen und Schüler im sozialen Vergleich nicht besonders gut oder schlecht abschneiden), was die mitunter sogar 
negativen Assoziationen zwischen dem mathematischen und sprachlichen Fähigkeitsselbstkonzept erklären mag. Empirisch ist das I/E-Modell in der Zwischenzeit sehr gut belegt: U.a. zeigten Möller, Pohlmann, Köller und Marsh (2009) in ihrer Metaanalyse von 69 Datensets (125'308 Schülerinnen und Schüler), dass modellkonform mathematische und sprachliche Leistungen hoch (.67), die entsprechenden Selbstkonzepte aber kaum korreliert waren (.10). Ebenso wiesen die Pfad-Koeffizienten zwischen den Leistungen und den Fähigkeitsselbstkonzepten im gleichen Fach jeweils positive Werte (.61 für Mathematik, .49 für Sprache) und für das jeweils andere Fach negative Werte auf (-21 für Leistungen in Mathematik und sprachliches Fähigkeitsselbstkonzept, -27 für sprachliche Leistungen und mathematisches Selbstkonzept). Dieses Zusammenhangsmuster zeigte sich in der Metaanalyse über Altersgruppen, Länder und das Geschlecht hinweg und unabhängig davon, ob Notenwerte oder Testleistungen herangezogen wurden. Ferner konnte theoriekonform belegt werden, dass das I/E-Modell lediglich für fachliche Selbstkonzepte Gültigkeit beanspruchen kann, nicht aber für das Konstrukt der Selbstwirksamkeit. So scheint die Genese von fachbezogenen Selbstkonzepten in hohem Maß auf querschnittlichen sozialen und dimensionalen Vergleichen zu beruhen, während Selbstwirksamkeitsüberzeugungen, wie von Bandura (1997, S. 80-81) postuliert, vor allem längsschnittlich aus Mastery-Erfahrungen resultieren. Wie bei den Selbstwirksamkeitsüberzeugungen ist auch bei fachlichen Kompetenzüberzeugungen davon auszugehen, dass sie in einem reziproken und sich wechselseitig verstärkenden Verhältnis zu Fachleistungen stehen: So zeigt sich in den letzten Jahren sowohl für den self-enhancement-Ansatz - bei Kontrolle bisheriger Leistungen führen hohe fachliche Selbstkonzepte über entsprechend produktives Lernhandeln zu subsequent guten bzw. sich günstig entwickelnden Fachleistungen und umgekehrt - als auch für den skill-development-Ansatz - die Leistungsergebnisse führen vermittelt über soziale Vergleichsprozesse (u.a bigfish-in-little-pond-Effekt, vgl. Abschnitte 3.2.2 und 3.3) und Attributionsprozesse (vgl. Abschnitt 5.3.3.1) zu entsprechenden Fähigkeitsselbstkonzepten - sowie für die Kombination der beiden Ansätze (vgl. Marsh \& Craven, 2006) deutliche empirische Evidenz (Überblick bei Marsh et al., 2012, S. 436-439). Mittlerweile besteht sodann Einigkeit darüber, dass - anders als ursprünglich von Shavelson et al. (1976) angenommen - der Selbstwert (self-esteem, self-worth bzw. general self-concept) als globale Einstellung gegenüber sich selbst - «I am a worthwhile person» (Harter, 2006, S. 509) - ebenso wenig mit Leistungsmaßen verknüpft ist wie nicht-akademische Komponenten des Selbstkonzepts (vgl. Marsh et al., 2012, S. 432-434). Zwar messen gerade Menschen mit hohem Selbstwert jenen 
Fähigkeitsbereichen hohen Wert bei, in denen sie sich auch als fähig wahrnehmen, allerdings prädiktieren diese domänenspezifischen Wichtigkeitsratings den globalen Selbstwert der Person kaum (z. B. Marsh, 1986a).

Die Charakteristik einer «extrem domain specificity» fachlicher Selbstkonzepte, die Marsh et al. (2012, S. 427) konstatieren, widerspiegelt sich in der Erwartungs-Wert-Theorie von Eccles, Wigfield und Kolleg*innen: Wie die Wert-Komponente konzipieren sie auch die Erwartungskomponente domänenbzw. aufgabenspezifisch. Empirisch hat sich dabei gezeigt (vgl. insb. Eccles \& Wigfield, 1995), dass Fähigkeitsüberzeugungen (ability perceptions), also gegenwartsbezogene Assessments eigener Kompetenzen zur Aufgabenmeisterung (z. B. «How good at math are you?»; S. 224) und Erfolgserwartungen (expectancy perceptions), also zukunftgerichtete Assessments der Wahrscheinlichkeit der Zielerreichung (z. B. «How well do you think you will do in your math course this year?»; S. 224) zwar konzeptionell unterschiedlich sind - bei ersterer handelt es sich um ein agent-means- bei letzterer um ein agent-ends-Konstrukt - , sich empirisch aber als hochgradig korreliert erweisen:

However, $[\ldots]$ empirical work has shown that children and adolescents do not distinguish between these two different levels of beliefs. Apparently, even though these constructs can be theoretically distinguished from each other, in real-world achievement situations they are highly related and empirically indistinguishable. (Eccles \& Wigfield, 2002, S. 119)

Als Folge davon werden in Untersuchungen Items zu beiden Facetten oft zu einer generellen expectancy for success-Skala kombiniert, die aber klar domänenspezifisch ist (vgl. Barron \& Hulleman, 2015, S. 504; vgl. Graham \& Weiner, 2012, S. 372). Eine große Zahl von längsschnittlichen Studien, die ability beliefs entlang dieser task-spezifischen Konzeption erhoben (zusf. Wigfield, Eccles, et al., 2015, S. 5; Wigfield et al., 2016, S. 60-61), belegen zumindest in westlichen Ländern konsistent einen Einbruch des durchschnittlichen Fähigkeitsselbstkonzepts bezüglich einer breiten Palette von schulischen Domänen ungefähr um das achte Lebensjahr und einen zweiten beim Übergang zur Sekundarstufe I (z. B. Buff \& Dinkelmann, 2012; Fredricks \& Eccles, 2002; Jacobs, Lanza, Osgood, Eccles \& Wigfield, 2002; Pesu, Aunola, Viljaranta \& Nurmi, 2016). Äußern sich Kinder in der frühen Kindheit im Durchschnitt recht optimistisch hinsichtlich ihrer Fähigkeiten, so scheinen sie einerseits entwicklungsbedingt zunehmend realistischer zu werden (erkennbar daran, dass die Korrelationen zu objektiven Leistungsmaßen zunehmen), andererseits dürften, wie oben bereits näher erläutert, soziale Vergleiche mit den Peers sowie Leistungsrückmeldungen von Lehrkräften und Eltern 
zunehmenden Einfluss auf ihre Fähigkeitseinschätzungen entfalten. So zeigen z. B. Spinath und Spinath (2005) in einer Studie mit 595 Kindern in Deutschland, dass die (allgemeinen) schulischen Selbstkonzepte der Kinder über die vierjährige Grundschulzeit zunehmend stärker - zwar auch mit Intelligenzmaßen - in höherem Grad aber mit den kindbezogenen Fähigkeitseinschätzungen ihrer Eltern und vor allem mit den durch ihre Lehrkraft erteilten Noten in Mathematik, Deutsch und im Sachfachunterricht assoziiert waren (wobei Spinath und Spinath in Einklang mit diesbezüglichen Befunden von Frome und Eccles (1998) vermuten, dass das elterliche Feedback als Mediator zwischen den Evaluationen der Lehrkraft und den Fähigkeitsselbstkonzepten der Kinder fungiert). Wie Wigfield, Eccles, et al. (2015, S. 5) in ihrer Zusammenfassung der Befundlage schreiben, zeigt sich sodann, dass bei der Gruppe derjenigen Kinder, die tiefe Notenwerte aufweisen, der Einbruch der domänenspezifischen Fähigkeitsselbstkonzepte generell am deutlichsten ausfällt. Wie oben bereits erläutert (vgl. Abschnitte 3.2.2 und 3.3), tritt in Bildungssystemen mit einem explicit between-school tracking - der leistungsbezogenen Verteilung der Lernenden auf verschiedene Schultypen - allerdings ein anderes Muster zutage: Erklärbar mit dem big-fish-little-pondEffekt brechen hier im Mittel die fachlichen Selbstkonzepte der Schüler*innen der anspruchsvollsten Schultypen stärker ein bzw. erhöhten sich diese bei den Lernenden im anforderungsärmsten Bildungsgang sogar (z. B. Chmielewski et al., 2013). Längsschnittlich angelegte Studien belegen ferner, dass sich die domänenspezifischen Fähigkeitsüberzeugungen über die Schuljahre stabilisieren. Bereits in der der Mitte der Primarstufe korrelieren sie bereits hoch über die Zeit (z. B. Buff et al., 2011; Spinath \& Steinmayr, 2008). Wigfield und Kolleg*innen vermuten, dass der Stabilitätsgrad bereits in der frühen Jugendzeit beträchtlich sein dürfte, was Interventionen zunehmend schwieriger mache (vgl. Wigfield, Eccles, et al., 2015, S. 6).

\subsubsection{Kausalitätsüberzeugungen}

Kausalitätsüberzeugungen (vgl. Abbildung 5.2) sind subjektive Kognitionen über den tatsächlichen oder lediglich vermuteten Einfluss verschiedener Mittel auf erwünschte oder unerwünschte Ergebnisse (vgl. Skinner, E. A., 1996, S. 552). 
Bei solchen auf «means-ends relations» bezogene Kognitionen ${ }^{6}$ geben sich Lernende rückwärtsgerichtet, beim Vorliegen von Leistungsergebnissen (vgl. Abbildung 5.1), Antworten auf die Fragen nach den möglichen Ursachen für einen Erfolg oder Misserfolg («Erfolg trat ein, weil in der Situation das zielführende Mittel A zur Verfügung stand»). Vorwärtsgerichtet, im Zuge der Initiierung von Handlungen, beinhalten sie Antworten auf die Frage nach dem Vorhandensein von effektiven Mitteln («Erfolg kann erwartet werden, weil in der Situation das zielführende Mittel vorliegt») (vgl. Pekrun, 1988, S. 67-70). Ausgehend vom locus of control-Konzept von Rotter (1982) stand in der Forschung zur Lern und Leistungsmotivation über Jahre die Frage im Zentrum, welche Klassen von Ursachen Individuen zur Erklärung von Leistungsergebnissen heranziehen (und welche ihnen unterliegenden kausalen Dimensionen für die differenziellen Effekte auf das nachfolgende Handeln und das emotionale Erleben verantwortlich sind. Gemäß Rotter unterscheiden Menschen zwischen einflussreichen Mitteln, die außerhalb und innerhalb ihrer selbst liegen:

\begin{abstract}
When a reinforcement is perceived by the subject as following some action of his own but not being entirely contingent upon his action, then, in our culture, it is typically perceived as the result of luck, chance, fate, as under the control of powerful others, or as unpredictable because of the great complexity of the forces surrounding him. When the event is interpreted in this way by an individual, we have labeled this a belief in external control. If the person perceives that the event is contingent upon his own behavior or his own relatively permanent characteristics, we have termed this a belief in internal control. (Rotter, 1966, S. 1, zit. nach Rotter, 1982, S. 266, Hervorhebungen im Original)
\end{abstract}

Kausalitätsüberzeugungen basieren demnach wesentlich auf dem Glauben von Menschen, wonach ganze Klassen von Lebenssituationen entweder durch eigenes Handeln bzw. durch eigene Persönlichkeitsmerkmale internal kontrolliert oder durch äußere Instanzen wie einflussreiche Individuen oder mehr oder weniger durchschaubare Strukturen external kontrolliert seien (vgl. Preiser \& Sann, 2010, S. 387). Zu letzteren gehören sog. Situations-Ergebnis-Erwartungen (situationoutcome expectancies) (vgl. Pekrun, 1988, S. 67-77; Pekrun, 2006, S. 318): Ein Individuum kann mit Blick auf die in einer Situation zur Verfügung stehenden Mittel und die zu erreichenden Ergebnisse zum Schluss kommen, dass sich letztere auch ohne eigenes Handeln einstellen (z. B. «Ich habe fleißige und fähige Mitarbeitende, die das Projekt auch ohne mein Dazutun zu einem erfolgreichen

\footnotetext{
${ }^{6}$ Breit rezipierte means-ends-Konstrukte sind u. a. «attributions», «contingency judgements», «locus of control», «response-outcome expectations» oder «strategy beliefs» (vgl. Skinner, E. A., 1995, S. 553).
} 
Ende bringen»), oder aber die Situation so beschaffen ist, dass sich negative Ergebnisse ergeben, wenn keine Gegenmaßnahmen ergriffen werden (z. B. «Wenn ich für dieses Examen nicht genug Zeit zum Lernen aufwende, werde ich durchfallen»). Im ersten Fall, in dem sich ein günstiges Ende auch ohne eigenes Handeln einstellt, dürfte die subjektive Kontrolle (bzw. die Erfolgserwartung) des Individuums hoch sein, im zweiten Fall, in dem eigenes Handeln zur Bewältigung der Aufgabe notwendig ist, stellt sich ihm die Frage nach den internalen Mitteln, und deren Funktionalität zur Erreichung des Ziels (vgl. unten: Kompetenzüberzeugungen). Im Anschluss an das locus of control-Konzept wurde eine Vielzahl von kausalen Dimensionen, die verschiedenen Klassen von Mitteln unterliegen, postuliert und empirisch bezüglich ihrer differentiellen Effekte geprüft: u. a. zeitliche Stabilität, Kontrollierbarkeit, Intentionalität, Globalität und Kontingenz (vgl. Skinner, E. A., 1996, S. 552). Im Lehr-Lern-Kontext hat Bernard Weiners Attributional Theory of Achievement Motivation and Emotion (1986, 1994, 2012), die differenzielle Wirkungen von Erfolgen und Misserfolgen in erster Linie in den drei Kausaldimensionen Lokus, Stabilität und Kontrollierbarkeit festmacht, bei Weitem am meisten Resonanz gefunden. Die Konzeption wird wegen ihrer Zweckmäßigkeit zur Erklärung von Wirkungen elterlichen evaluativen Feedbacks in den folgenden beiden Kapiteln näher beleuchtet.

\subsubsection{Ursachenzuschreibungen - Weiners attributionale Theorie der Leistungsmotivation (1986)}

Attributionen bezeichnen «Ursachen, die Individuen zur Erklärung von Ereignissen, Handlungen und Erlebnissen (genereller: Effekten) in verschiedenen Lebensbereichen heranziehen» (Försterling, 1986, S. 23) und sind damit grundsätzlich rückwärtsgerichtete means-ends-Kognitionen (z. B. «Ich habe an der Prüfung einen Misserfolg errungen, weil ich mich zu schlecht vorbereitet habe»). Mit Blick auf den zeitlichen Ablauf im von Skinner (1995, S. 20) skizzierten Kompetenzsystem (vgl. Abbildung 5.1) evaluiert das Individuum gemäß Weiner nach Abschluss einer Handlung zuerst, wie das Ergebnis zu bewerten ist (Erfolg oder Misserfolg), bevor es sich vor allem bei negativen, ungewöhnlichen und unerwarteten sowie wichtigen Ergebnissen (vgl. Möller \& Köller, 2000; Stiensmeier-Pelster \& Heckhausen, 2010, S. 396; Wong \& Weiner, 1981) warum-Fragen stellt und eine Suche nach den Ursachen einleitet. Welche konkrete Ursache das Individuum schließlich in der causal search findet, hängt von seinen Leistungserfahrungen und den Informationen ab, die ihm - u. a. aus dem Feedback anderer - zum spezifischen Handlungsergebnis zur Verfügung stehen, aber auch davon, wie es diese Informationen mit Hilfe kausaler Schemata, also Überzeugungen zu Ursache-Wirkungs-Zusammenhängen, entlang des 
Kovariationsprinzips (vgl. Kelley, 1973; Kelley \& Michela, 1980) ${ }^{7}$ sowie unter dem Einfluss selbstwertdienlicher Verzerrungen (vgl. unten) und anderen Analysefehlern $^{8}$ kombiniert (vgl. Schunk et al., 2014, S. 90; Weiner, 1994). Je nach subjektiv wahrgenommenen Gründen empfindet es leistungsbezogene Emotionen, zieht Schlüsse darüber, inwieweit ähnliche Handlungen in Zukunft realisierbar und wünschbar seien bzw. wie hoch die eigene Kontrolle über das Ereignis zu veranschlagen sei (vgl. Weiner, 1985, S. 565).

Hervorzuheben gilt, dass sich Attributionstheorien somit nicht damit befassen, was die tatsächlichen Ursachen von Ereignissen und Handlungen sind, sondern auf die subjektiven idiosynkratischen Erklärungen und Begründungen fokussieren $^{9}$, mit denen Individuen sich selbst oder anderen gegenüber operieren: «Attribution Theory is primarily concerned with naive psychological theories, that is, how 'the man on the street' explains his own or other individuals' behaviours and behavioural outcomes; therefore attribution theory makes ,naive psychology' its topic» (Försterling, 2001, S. 4). Wie es die Darstellung des Kompetenzsystems in Abbildung 5.1 nahelegt, üben die konkreten Zuschreibungen sodann keinen direkten Einfluss auf die Motivation und das Handeln aus, sondern beeinflussen diese Prozesse indirekt über ihre Wirkung auf Kontrollüberzeugungen bzw. Ziele, Wertüberzeugungen und Emotionen (Perry \& Hamm, 2017, S. 62; Schunk et al., 2014, S. 81).

Leistungsergebnisse, die in den rund sechzig Jahren seit Fritz Heiders Monografie «The Psychology of Interpersonal Relations» (1958) die Dreh- und Angelpunkte der Forschung zu Attributions- und attributionalen Prozessen ${ }^{10}$

\footnotetext{
${ }^{7}$ Bei einer strikt rationalen Vorgehensweise in der kausalen Suche müssten Individuen laut Kelley (1973, S. 159) auf folgende drei Informationsquellen fokussieren: auf die Distinktheit (Wie spezifisch reagiert ein Akteur auf einen bestimmten Stimulus?), auf den Konsensus (Reagieren andere auch in ähnlicher Weise auf diesen Stimulus?) und auf die Konsistenz (Ist die Reaktion des Akteurs auf diesen Stimulus zu verschiedenen Zeitpunkten die gleiche?). Ist z. B. die Distinktheit und der Konsensus niedrig, die Konsistenz aber hoch, wird mit hoher Wahrscheinlichkeit auf dispositionale Merkmale bzw. auf die Begabung der Person als Ursache geschlossen (vgl. Möller, 2018, S. 30).

${ }^{8}$ Gemäß Ross (1977) neigen Menschen dazu, bei andern den Einfluss dispositionaler Faktoren auf deren Verhalten zu über- und denjenigen situationaler Faktoren zu unterschätzen (fundamental attribution error). Laut Jones und Nisbett (1971) zeigen sie sodann die Tendenz, das eigene Handlungsergebnis auf externale und das Handlungsergebnis anderer auf internale Faktoren zurückzuführen (actor-observer effect).

${ }^{9}$ Weiner (1994, S. 270) spricht von «phänomenologischer Kausalität», die das Beschäftigungsfeld von Attributionstheorien darstellten.

${ }^{10}$ Attributionstheorien untersuchen die Frage, unter welchen Bedingungen verschiedene Kausalzuschreibungen zustande kommen: Welche Informationen werden hinzugezogen? Wie
} 
waren, werden von Menschen mitunter auf ihre stabilen und zeitweiligen affektivmotivationalen Zustände, auf die Schwierigkeit der Domäne bzw. der Aufgaben, auf die Hilfe oder Beeinträchtigung durch andere Menschen sowie auf Glück oder Pech zurückgeführt (vgl. Schunk et al., 2014, S. 97; Weiner, 1994, S. 269274). Zumindest im westlichen Kulturkreis, so Graham und Taylor (2016), werden Erfolge und Misserfolge in schulischen Leistungssituationen aber meistens den Ursachenfaktoren Fähigkeit/Begabung sowie konstanter oder zeitweiser Anstrengung zugeschrieben:

When explaining achievement outcomes, individuals attach the most importance to their perceived competence and how hard they try. That is, when someone succeeds, he or she probably infers that 'I tried hard' or 'I am smart' and if the person does not succeed, he or she is likely to conclude that 'I did not try hard enough' or 'I am not very smart!' (Graham \& Taylor, 2016, S. 13)

Die inhaltlich vielgestaltigen Ursachen, die Menschen als Erklärungen heranziehen, entfalten ihre Wirkung auf Kontroll- und Wertkognitionen bzw. Emotionen ${ }^{11}$ über eine Reihe von Charakteristika, die ihnen gemeinsam sind - «the basic properties of these causes» - und wie diese gemeinhin interpretiert werden (Weiner, 1985, S. 549). In seiner attributionalen Theorie intrapersonalen Verhaltens postuliert Weiner $(1985,2005,2012)$, dass hierbei insbesondere drei Kausaldimensionen relevant seien:

- Die Dimension Lokus (causal locus), von Heider (1958) bzw. Rotter (1982) übernommen (vgl. Abschnitt 5.3.3), bezieht sich auf die Verortung der Ursache und besteht aus den beiden Polen internal und external («weil es an mir liegt» vs. «weil es an den äußeren Umständen bzw. anderen Personen liegt»).

- Die Dimension Stabilität (causal stability) verweist auf die Veränderlichkeit der Ursachen: Sie treten entweder zeitlich stabil oder variabel auf («weil ich

werden die Informationen bei der Zuschreibung gewichtet? Attributionale Theorien widmen sich demgegenüber der Frage, welche Wirkung bestimmte Ursachenzuschreibungen auf das darauffolgende Handeln und Erleben des Individuums entfalten (vgl. Schunk et al., 2014, S. 83; Stiensmeier-Pelster \& Heckhausen, 2010, S. 390).

${ }^{11}$ In der Attributionstheorie Weiners, die sich im weiteren Sinne als Erwartungs-Wert-Theorie versteht (vgl. Weiner, 2012, S. 136-140), erscheint die Wert-Komponente nicht als detailliert ausgearbeitete Komponente, sondern wird in den Emotionen eingefangen, die spontan durch die Ergebnisse (Freude, Trauer) oder durch die Ursachenzuschreibungen evoziert werden: Stolz, Scham, Schuld etc. In Kombination mit den Erfolgserwartungen prädiktieren die Emotionen behaviorale Aspekte: Das Wahlverhalten, das Engagement, die Persistenz sowie Leistungsergebnisse (vgl. Schunk et al., 2014, S. 105). 
ständig so bin oder handle» bzw. «weil die Umstände immer so sind» vs. «weil ich manchmal so bin oder handle» bzw. «weil die Umstände dieses Mal so waren»).

- Die Dimension Kontrollierbarkeit (causal controllability) bezieht sich auf die volitionale Beinflussbarkeit des Ursachenfaktors: Lässt sich der Grund für das Ergebnis durch das Individuum ändern («weil es durch mich beeinflussbar ist» vs. «weil es nicht meinem Willen unterliegt»)? ${ }^{12}$ Kontrollierbarkeit ist, wie Weiner erkennt, nicht gänzlich unabhängig von der Lokus- und Stabilitätsdimension:

Ist zum Beispiel eine Ursache sowohl internal als auch stabil (wie Fähigkeit), dann wird sie in der Regel auch als unkontrollierbar angesehen. Es gibt jedoch einige Ursachenfaktoren (wie z.B. momentane Stimmung und Müdigkeit), welche internal und variabel sind, jedoch nicht unter willentlicher Kontrolle stehen, während Anstrengung zwar ebenfalls internal und instabil, aber kontrollierbar ist. Daher scheint es vernünftig, Kontrollierbarkeit als dritte Dimension der Kausalität zu berücksichtigen. (Weiner, 1994, S. 271)

Jede Ursache lässt sich entlang der drei Kausaldimensionen im Achtfelderschema Weiners (1994, S. 271) (vgl. Tabelle 5.1) einordnen:

For example, ability is typically perceived as internal, stable, and uncontrollable. When we attribute our failure to low ability, we tend to see this as a characteristic of ourselves, enduring over time, and beyond personal control. Effort, on the other hand, is also internal, but unstable and controllable. Failure attributed to insufficient effort indicates a personal characteristic that is modifiable by one's own volitional behavior. (Graham \& Taylor, 2016, S. 13)

Ursachen der jeweils gleichen Zelle, so unterschiedlich sie inhaltlich sein mögen, führen gemäß Weiner stets zu denselben psychologischen Konsequenzen (vgl. Weiner, 1985, S. 566).

Die Dimension Lokus übt vor allem einen Einfluss auf den Selbstwert (selfworth, self-esteem) und entsprechende Emotionen aus (vgl. Abschnitt 5.3.2.2

\footnotetext{
${ }^{12}$ Weiner grenzt Kontrollierbarkeit von Intentionalität ab, die verschiedentlich auch als Kausaldimension vorgeschlagen wurde (vgl. Weiner, 1985, S. 566). Intentionalität impliziere ein Bedürfnis oder ein Wunsch, Kontrollierbarkeit dahingegen nicht. Dass es sich um zwei getrennte Dimensionen handle, sei zum Beispiel an der folgenden Aussage erkennbar: «Ich will mit dem Trinken aufhören, aber ich kann mich nicht kontrollieren». Die Absicht sei vorhanden, die Kontrollierbarkeit aber gering (vgl. Weiner, 1994, S. 271). Das Beispiel macht deutlich, dass sich der Ausdruck Steuerbarkeit als Synonym für Kontrollierbarkeit anbietet (vgl. Stiensmeier-Pelster \& Heckhausen, 2010, S. 392).
} 
und 5.5.1.4): Erfolge, die internal eigenen Fähigkeiten, Persönlichkeitsmerkmalen, eigener Anstrengung und Aufmerksamkeit oder dem eigenen Strategieeinsatz zugeschrieben werden, evozieren in höherem Maß die aktivierende Emotion Stolz (pride), als wenn diese external auf die situativen Umstände oder das Handeln anderer zurückgeführt werden. Umgekehrt werden die negativ-valenten aktivierenden Emotionen Scham und Schuld intensiver erlebt, wenn Misserfolge internalen Faktoren zugeschrieben werden (vgl. Graham \& Taylor, 2016, S. 18; Pekrun \& Perry, 2014, S. 121). Zur Steigerung, aber auch zum Schutz des Selbstwerts (vgl. Covington, 1992, S. 74-103) weisen Individuen bereits bei der intrapersonalen Ursachensuche einen hedonic bzw. self-serving bias (vgl. Miller \& Ross, 1975) auf - sie zeigen die Tendenz, Erfolge eher internal und Misserfolge eher external zuzuschreiben -, und setzen bei Misserfolgen zum Zweck der Gesichtswahrung gegenüber Kommunikationspartnern mitunter auf die Strategie, externale Aspekte als ursächlich zu deklarieren - z. B. «leistungsstarke Klasse», «Einstellung der Lehrkraft» (vgl. Tabelle 5.1) (ausführlicher: vgl. Abschnitt 5.5.1.4). Gleichermaßen setzen auch Dritte - z. B. Lehrkräfte, Eltern oder Peers - die sich des self-serving bias in der Ursachenzuschreibung des Gegenübers durchaus bewusst sind (vgl. McAllister, 1996) zur Gesichtswahrung, zur Selbstwertförderung und zum Trost das Mittel einer Attribuierung auf externale Ursachen ein («Du solltest auch nicht außer Acht lassen, dass diese Aufgaben sehr schwer waren, der Notenschnitt war tief»). Mitunter weigern sie sich allerdings auch, affirmativ auf die selbstwertdienliche Rechtfertigungsstrategie des Betroffenen einzutreten («Nein, es lag an deiner fehlenden Anstrengung!») (vgl. Schunk et al., 2014, S. 106).

Die Dimension Stabilität wirkt sich vor allem auf die Kontrollüberzeugungen bzw. die Erfolgserwartung des Individuums aus (vgl. Weiner, 1985, S. 566). Werden Erfolge oder Misserfolge auf einen stabilen Faktor attribuiert (Fähigkeit, Talent oder habituelle Handlungsmuster, vgl. Tabelle 5.1), so ist die Wahrscheinlichkeit hoch, dass sie wieder auftreten, was im Erfolgsfall mit Zuversicht bzw. der positiv-valenten, aktivierenden Emotion Hoffnung (hope) und im Misserfolgsfall mit der negativ-valenten, deaktivierenden Emotion Hoffnungslosigkeit (hopelessness) bzw. bei gehäuftem Auftreten mit erlernter Hilflosigkeit (learned helplessness) einhergeht (vgl. Abschnitt 5.3.1). Wird auf variable Faktoren wie Anstrengung, insbesondere aber auf unkontrollierbare Ursachen wie Glück und Pech oder Krankheit (vgl. Tabelle 5.1) attribuiert, so schwankt das Individuum zwischen Hoffnung (hope) und Ängstlichkeit (anxiety), zwei aktivierenden, aber entgegengesetzt valenten emotionalen Zuständen und entsprechenden Folgen für 
Tabelle 5.1 Beispiele von Ursachenzuschreibungen nach einem Misserfolg im dreidimensionalen Klassifikationsschema nach Weiner (1994, S. 271): Lokus, Stabilität und Kontrollierbarkeit (vgl. Perry \& Hamm, 2017, S. 63; Adaptionen E.S.)

\begin{tabular}{l|l|l|l|l}
\hline & \multicolumn{2}{l|}{ internal } & \multicolumn{2}{l}{ external } \\
\cline { 2 - 5 } & stabil & variabel & stabil & variabel \\
\hline kontrollierbar & $\begin{array}{l}\text { weil ich mich } \\
\text { wie immer zu } \\
\text { wenig } \\
\text { vorbereitet } \\
\text { habe }\end{array}$ & $\begin{array}{l}\text { weil ich mich } \\
\text { nicht auf diese } \\
\text { Prüfung } \\
\text { vorbereitet } \\
\text { habe }\end{array}$ & $\begin{array}{l}\text { weil die Lehrerin } \\
\text { etwas gegen } \\
\text { mich hat }\end{array}$ & $\begin{array}{l}\text { Feil meine } \\
\text { Freunde mir } \\
\text { dieses Mal nicht } \\
\text { geholfen haben }\end{array}$ \\
\hline unkontrollierbar & $\begin{array}{l}\text { weil ich für } \\
\text { dieses Fach } \\
\text { völlig } \\
\text { untalentiert } \\
\text { bin }\end{array}$ & $\begin{array}{l}\text { weil ich an } \\
\text { jenem Tag } \\
\text { Migräne hatte }\end{array}$ & $\begin{array}{l}\text { weil ich in einer } \\
\text { sehr } \\
\text { leistungs-starken } \\
\text { Klasse bin }\end{array}$ & $\begin{array}{l}\text { weil ich dieses } \\
\text { Mal einfach Pech } \\
\text { mit den } \\
\text { Prüfungsaufgaben } \\
\text { hatte }\end{array}$ \\
\hline
\end{tabular}

Lernen und Leisten ${ }^{13}$ (vgl. Graham \& Taylor, 2016, S. 18; Pekrun, 2006, S. 320; Pekrun \& Perry, 2014, S. 121; Weiner, 1985, S. 566).

Die Dimension Kontrollierbarkeit löst beim Individuum Folgerungen zur Verantwortlichkeit (responsibility inferences) für ein Ergebnis aus: «One is responsible (able-to-respond) for not expending effort, but not for lacking aptitude, which cannot be changed merely by willpower» (Weiner, 2012, S. 145). Fühlt man sich selber verantwortlich, werden die oben bereits angesprochenen selbstgerichteten Emotionen Scham (shame) und Schuld (guilt) empfunden, werden

\footnotetext{
${ }^{13}$ Pekrun und Kolleg*innen (vgl. Pekrun, Frenzel, Goetz \& Perry, 2007; Pekrun \& Götz, 2006; Pekrun \& Perry, 2014; Pekrun \& Stephens, 2010) postulieren in der Control-Value Theory of Achievement Emotions, dass die Charakteristiken von Emotionen folgende Wirkungen auf die Leistungsmotivation entfalten: Positiv aktivierende Emotionen sind motivationsförderlich, weil sie handlungsverstärkend (Freude), wertsteigernd (Stolz) oder kontrollförderlich (Hoffnung) wirken. Negativ deaktivierende Emotionen sind motivationshinderlich, weil sie mit geringen Kontrollüberzeugungen (Hoffnungslosigkeit) oder geringen Anreizen (Langeweile) assoziiert sind. Positiv deaktivierende Emotionen wie z. B. Erleichterung vermindern die unmittelbare Leistungsbereitschaft, können sich aber verstärkend bezüglich eines längerfristigen Engagements auswirken. Negativ aktivierende Emotionen wie z. B. Ängstlichkeit und Scham untergraben die Interessenbildung bzw. die intrinsische Motivation, evozieren aber eine starke extrinsische Motivation, vorangegangene Misserfolge nicht erneut zu erleben. Mit anderen Worten sind positiv deaktivierende Emotionen und negativ aktivierende Emotionen bedeutend komplexer und variabler in ihrer Wirkung auf die Leistungsmotivation als erstere (vgl. Pekrun \& Perry, 2014, S. 132).
} 
dahingegen andere für einen Erfolg oder Misserfolg verantwortlich gemacht, werden die fremdgerichteten Emotionen Dankbarkeit (gratitude) bzw. Ärger (anger) evoziert (vgl. Graham \& Taylor, 2016, S. 22; Pekrun, 2006; Weiner, 1985, S. 565). Ursachen für einen Misserfolg in unkontrollierbaren Ursachen finden («ich bin unbegabt», «ich hatte Migräne», «ich bin in einer leistungsstarken Klasse», «ich hatte Pech», vgl. Tabelle 5.1) bedeutet ein Referieren auf strukturelle und somit schwer veränderbare Faktoren in einem selber oder in der Umwelt («so bin ich halt» bzw. «so ist/war es halt») und reduziert nicht nur die persönliche Verantwortlichkeit für das Ergebnis, sondern auch die Motivation, etwas daran zu ändern («es bringt ja doch nichts»). Willentlich beeinflussbare Ursachen für Misserfolge, so zeigt sich bei einem Blick auf Tabelle 5.1, sind dahingegen solche, die auf das eigene oder das Handeln anderer - also prozessuale Aspekte - verweisen. Selbst wenn man das hinderliche Handeln anderer bzw. die zugrundeliegenden Überzeugungen und Haltungen verantwortlich macht («weil die Lehrerin etwas gegen mich hat und mich benachteiligt», «weil meine Freunde mir dieses Mal nicht geholfen haben»), besteht hierbei die Möglichkeit, im Sinne von «volitional change» (Weiner, 2012, S. 144) aktiv auf diese Prozesse Einfluss zu nehmen und die Bedingungen des zukünftigen Leistungsereignisses zu optimieren (sich z. B. an die Lehrerin wenden und den Eindruck einer ungerechten Behandlung ansprechen bzw. vor der nächsten Prüfung früh die Peers kontaktieren, um Termine für das gemeinsame Lernen festzulegen $)^{14}$.

Wie Weiner (2012) im Rahmen seiner attributionalen Theorie interpersonalen Verhaltens postuliert, übt die Dimension Kontrollierbarkeit wegen des impliziten Aspekts der Verantwortlichkeit denn auch bei der Attribuierung von Erfolg und Misserfolg durch Dritte einen entscheidenden Einfluss auf deren emotionale und behaviorale Reaktion aus: Kommen sie in ihrer kausalen Suche nach einem Misserfolg des Kindes zum Schluss, dass das Ergebnis an dessen fehlenden Anstrengung, also an einer willentlich kontrollierbaren Ursache, lag und erkennen sie keine lindernden Umstände, so machen sie das Kind verantwortlich und reagieren mit Ärger, einer Emotion, die wiederum ablehnende Verhaltensweisen, Tadel und Strafe begünstigt. Mitgefühl oder Mitleid und entsprechende Verständnisbekundungen und Hilfsangebote durch das soziale Umfeld resultieren dahingegen bei einer Zuschreibung des Misserfolgs auf nichtkontrollierbare Ursachen wie fehlende Begabung (vgl. Graham \& Taylor, 2016, S. 22; Weiner, 2012).

\footnotetext{
${ }^{14}$ Zur Kontroverse um die Frage, ob eine Ursache, die außerhalb einer Person angesiedelt sei, durch diese kontrollierbar sei, vgl. Schunk et al. (2014, S. 101).
} 


\subsubsection{Empirische Befunde zur Funktionalität von Attributionen und von attributionalem Feedback}

Die Befunde empirischer Studien im Bereich der Lern- und Leistungsmotivation, die in den letzten vierzig Jahren entstanden sind, stützen generell die Annahmen der intra- und interpersonalen Attributionstheorie Weiners bezüglich der Konsequenzen der drei Kausaldimensionen auf Kontrollkognitionen und Emotionen sowie subsequent auf Engagement, Persistenz und Leistung (zsf. Dresel, 2004, S. 46-65; Graham \& Taylor, 2016; Stiensmeier-Pelster \& Heckhausen, 2010, S. 417-421; Weiner, 1986, 2012). Namentlich Unterschiede in der Stabilität und der Kontrollierbarkeit, die Individuen bei Anstrengungs- und Fähigkeitsattributionen wahrnähmen, so fassen Perry und Hamm (2017) zusammen, seien bewiesenermaßen für eine Reihe von motivationalen und behavioralen Folgen in Leistungssituationen verantwortlich:

Although both causes are internal, ascribing poor performance to low ability (stable, uncontrollable) decreases motivation, whereas low effort (unstable, controllable) increases motivation. Lack of effort, bad strategy, or poor note taking are controllable causes often ascribed for failure, but because they can be altered by trying harder, using a better strategy, or taking clearer notes, they can increase motivation and performance. External (uncontrollable) causes, such as bad luck, poor teaching, or test difficulty, may create less negative affect and are less harmful to pride and self-esteem, but they are likely to impair motivation nonetheless. (Perry \& Hamm, 2017, S. 63)

Bezüglich der Funktionalität einer Ursachenzuschreibung auf Fähigkeit oder Anstrengung im Hinblick auf kommende Lern- und Leistungssituationen - demjenigen Aspekt, der mit Blick auf elterliche evaluative Feedbacks besonders interessiert - fällt die Befundlage im Misserfolgsfall recht eindeutig aus (vgl. Dresel, 2004, S. 64-65; Dresel \& Lämmle, 2017, S. 114): Je stärker bei einem Misserfolg nicht nur auf variable Faktoren attribuiert wird, sondern auf solche, die auch kontrollierbar sind, desto höher fällt generell die Erfolgserwartung aus. Die Attribution auf fehlende Anstrengung oder ungenügenden Strategieeinsatz kann nach Misserfolgen sodann vor dem Absinken des fachlichen Fähigkeitsselbstkonzepts und des Selbstwerts bewahren (z. B. Skaalvik, 1994), wohingegen die wiederholte Attribution auf fehlende Fähigkeit bzw. Begabung in einer (zu) pessimistischen Einschätzung eigener Handlungsmöglichkeiten bzw. in Hilf- und Hoffnungslosigkeit resultieren kann (vgl. Stiensmeier-Pelster \& Heckhausen, 2010, S. 420-421). Die Zuschreibung eines Misserfolgs auf externale Ursachen (z. B. Pech), wirkt sodann tatsächlich selbstwertwahrend. Allerdings besteht Einigkeit darüber, dass 
solche Attributionsmuster, «[...] als wenig motivationsförderlich bezeichnet werden [sollten], da sie keine zukünftigen Anstrengungen implizieren» (Dresel, 2004, S. 64).

Aus dieser Befundlage lassen sich bei Misserfolgen mit Blick auf die schematische Darstellung des Kompetenzsystems von Skinner (1995, S. 20) (vgl. Abbildung 5.1) zwei generelle Attributions-Emotions-Motivations-PerformanzPfade (attribution-based paths) ausmachen (vgl. Perry \& Hamm, 2017), die sich hinsichtlich ihrer Funktionalität deutlich unterscheiden:

- Auf dem maladaptiven Pfad wird ein Misserfolg auf die unkontrollierbare, stabile, internale Ursache fehlende Begabung/Fähigkeit zurückgeführt, was mit einer geringen Kontrolleinschätzung und entsprechenden negativen Emotionen und unliebsamen Effekten auf den Selbstwert einhergeht, woraus wiederum ungünstige motivationale Zustände und entsprechend ungenügende Bemühungen resultieren, welche letzten Endes in erneute Misserfolge münden (vgl. Perry \& Hamm, 2017, S. 71). Letztere wirken dann wiederum ungünstigstabilisierend auf die Attributionsprozesse zurück (vgl. Kelley \& Michela, 1980).

- Auf dem adaptiven Pfad wird ein Misserfolg auf kontrollierbare, variable Faktoren - fehlende Anstrengung, schlechte Strategien oder geringe Aufmerksamkeit - attribuiert, was mit erhöhten Kontrolleinschätzungen («ich kann es in den Griff kriegen») und entsprechenden Affekten einhergeht, die wiederum zu günstigeren motivationalen Zuständen führen, welche die Wahrscheinlichkeit eines Erfolgs und günstige Folgerungen bei der kausalen Suche erhöhen (vgl. Perry \& Hamm, 2017, S. 71).

Experimentelle oder quasiexperimentelle Studien zu Reattributionstrainings (attributional retraining programs) - im Labor oder in Schule und Unterricht durchgeführte Interventionen, die darauf abzielen, betroffene Kinder, Jugendliche (zsf. Schunk et al., 2014, S. 115-119) oder junge Erwachsene (zsf. Perry \& Hamm, 2017) mittels verbalem oder schriftlichem attributionalem Feedback oder videobasierten Modellierungstechniken von einem maladaptiven Pfad abzubringen -, belegen nicht nur, dass mittels solchen Feedbacks erfolgreich von außen in den Zuschreibungsprozess Einfluss genommen und ein funktionaler Attributionsstil mit den genannten Konsequenzen initiiert werden kann, sondern auch, unter welchen Bedingungen solche Interventionen die intendierten Wirkungen entfalten (zsf. Dresel, 2004, S. 66-89; Ziegler \& Finsterwald, 2008). Wie die Übersichtsdarstellung bei Perry und Hamm (2017) zeigt, erweisen sich in 
Misserfolgssituationen zumindest bei Universitätsstudierenden folgende vier attributionsbasierten Interventionsstrategien ( $a, b$, c oder d) bezüglich ausgewählter Kriterien als wirksam:

- Zur Förderung eines Attributionsstils, der nach Misserfolgen generell auf kontrollierbaren Ursachen (internal oder external sowie stabil oder variabel) oder aber auf internal, variabel und kontrollierbaren Ursachen beruht, erwiesen sich die Strategien a) Betonen kontrollierbarer Ursachen (ungenügende Anstrengung, schlechte Lernstrategien, fehlende Aufmerksamkeit) oder b) das Herunterspielen unkontrollierbarer Ursachen sowie c) die Kombination aus beidem als erfolgreich (vgl. Perry \& Hamm, 2017, S. 76).

- Zur Förderung günstiger emotionaler Zustände (z. B. mehr Lernfreude, Hoffnung oder Stolz, weniger Langeweile, Scham oder Hilflosigkeit) erwiesen sich nach erlebten Misserfolgen bzw. bei ursprünglich pessimistischen Studierenden a) das Betonen kontrollierbarer Ursachen sowie c) die Kombination aus Betonen kontrollierbarer Ursachen und gleichzeitigem Herunterspielen unkontrollierbarer Ursachen als produktiv (vgl. Perry \& Hamm, 2017, S. 76).

- Signifikant bessere Testergebnisse oder Noten als die Kontrollgruppe sowie positive Effekte auf die Persistenz ließen sich in Feldstudien, die sich z. T. über mehrere Semester erstreckten, bei wenig zuversichtlichen Studierenden durch die Strategien a) Betonen kontrollierbarer Ursachen sowie c) Kombination aus Betonen kontrollierbarer Ursachen und gleichzeitigem Herunterspielen unkontrollierbarer Ursachen nachweisen. In mehreren Laborstudien konnten sodann Leistungsgewinne nachgewiesen werden, wenn die Strategie d) dimensionale Eigenschaften der Ursachen verändern («Fähigkeit ist unstabil und kann durch beharrliche Anstrengung verbessert werden!») eingesetzt wurde (vgl. Perry \& Hamm, 2017, S. 77).

Auf der Basis ihrer Metaanalyse von 58 attributionsbezogenen Interventionsstudien, die zwischen 1980 und 2006 durchgeführt wurden, werfen Ziegler und Finsterwald (2008) ferner ein Licht auf die Frage, welche spezifische Ursachenangabe sich bei Feedback, das auf kontrollierbare internale Gründe abhebt, als besonders produktiv herausstellt. Sie kommen zum Schluss, dass sich bei Misserfolgen speziell «[...] das attributionale Feedback, nicht ausreichende beziehungsweise falsche Lernstrategien angewandt zu haben, als sehr positiv [erweist]» (Ziegler \& Finsterwald, 2008, S. 425). Schunk et al. (2014) raten bezüglich dieser Frage auf der Basis eigener Forschung (vgl. Schunk, 1984; Schunk \& Rice, 1996), vor allem darauf zu achten, ein akkurates Feedback zu geben, um in den Augen der Lernenden glaubwürdig zu erscheinen (vgl. Abschnitt 5.7): 
One suggestion is to attribute all failures to low effort and encourage students to make this low effort attribution. This often is good advice; it communicates to students that they can do better because effort is an unstable, internal, and controllable cause that students can change. However, there are occasions when students work hard and still perform poorly because they lack the skills or knowledge for the task. In these situations, students know they worked hard, and to be told by the teacher [or the parent] to keep trying harder can be frustrating and lead to a discounting of [this person's] feedback. It would be more accurate to point out to students the skills or knowledge that they lack, communicate that skills and knowledge can be learned, and then teach these skills and knowledge. (Schunk et al., 2014, S. 118)

Auch Dresel (2004, S. 78-79) rät aufgrund von Befunden, wonach die ausschließliche Darbietung von Anstrengungsattributionen nach Misserfolgen von begrenzter Effektivität sei, weniger die Quantität, sondern die Qualität der Anstrengung zu betonen und im Sinne einer Strategievermittlung zusätzlich zum Feedback Hinweise zu geben, wie die Qualität des Lernens gesteigert werden könnte.

Im Erfolgsfall ist die Handlungsfunktionalität von Fähigkeits- und Anstrengungsattributionen weit weniger eindeutig (vgl. Dresel, 2004, S. 64-65). Während Einigkeit darüber besteht, dass bei Erfolgen die Ursache grundsätzlich bei internalen Faktoren festgemacht werden sollte - externale Zuschreibungen zeitigen in diesem Fall weder auf den Selbstwert noch auf Kontrollüberzeugungen positive Effekte -, und sich Attributionen auf Fähigkeit und Begabung grundsätzlich positiv auf das Fähigkeitsselbstbild auswirken, ist bis dato ungeklärt, ob wegen des Aspekts der Unkontrollierbarkeit dieses Ursachenfaktors mit einer reduzierten Anstrengungsbereitschaft zu rechnen ist. Nimmt man Erfolge als stabil wahr, kann man seinen Aufwand vor Leistungsereignissen reduzieren und braucht keine Ressourcen in die Entwicklung ausgefeilterer Lern- und Arbeitsstrategien zu investieren. Dies könnte sich gerade nach Übertritten im Kontext von Bezugsgruppeneffekten negativ auswirken, wenn sich Kinder und Jugendliche in Folge der Selektion in einer leistungshomogeneren Klasse wiederfinden, in der nun ein Anforderungsniveau herrscht, welches von ihnen ein erhöhtes Engagement und entsprechende selbstregulativen Techniken verlangt (vgl. Abschnitt 3.2). Auch bezüglich der affektiven Konsequenzen von Fähigkeits- vs. Anstrengungsursachen sind die Befunde uneindeutig, wie Dresel festhält: «[Es] kann lediglich als gesichert gelten, dass Fähigkeitszuschreibungen ein Gefühl von Kompetenz und Anstrengungsattributionen ein Gefühl von Tüchtigkeit auslösen» (Dresel, 2004, S. 65). Anders als Fähigkeitsattributionen gelten Anstrengungsattributionen bei einem Erfolg gemeinhin aber als selbstwertgefährdend («Ich bin im Vergleich zu meinen Klassenkameradinnen und -kameraden leider jemand, der für einen Erfolg ständig hartes Arbeiten nötig hat»). 
Grundsätzlich, so wird deutlich, dürfte also die Art der Verknüpfung der beiden Ursachenfaktoren in der kausalen Suche zentral sein (vgl. Dresel, 2004, S. 65; Kelley, 1973): Problematisch erscheinen auf der Basis des eben Geschilderten vor allem kompensatorische Verknüpfungen («Ich habe mich für diesen Erfolg im Vergleich zu meinen Klassenkamerad*innen stark bemühen müssen, also bin ich wohl nicht so begabt» bzw. «Ich musste mich im Vergleich zu meinen Klassenkamerad*innen kaum anstrengen. Da ich offenbar so begabt bin, ist besondere Anstrengung auch weiterhin nicht notwendig»). Bei positiv kovariierenden Verknüpfungen («wegen meinen durch Anstrengung besser gewordenen Fähigkeiten» bzw. «wegen meinen Fähigkeiten, die sich durch Anstrengung noch laufend erweitern») - wenn Individuen also eine incremental theory of intelligence bzw. eine mastery goal orientation (vgl. Blackwell et al., 2007; Hong et al., 1999) erkennen lassen (vgl. Abschnitt 4.2.1.2), dürften dahingegen weder der negative Effekt, den Fähigkeitsattributionen potentiell auf die Anstrengungsbereitschaft ausüben, noch die selbstwertgefährdenden Implikationen, die mit Anstrengungsattributionen nach Erfolgen einhergehen, eine Rolle spielen (vgl. Dresel \& Ziegler, 2006, S. 51).

In seiner Studie zu attributionalem Feedback, welches 545 Siebtklässler*innen im Rahmen der Bearbeitung einer Mathematiklernsoftware in sechs Sitzungen dargeboten wurde, konnte Dresel (2004) belegen, dass wenn kontinuierlich entweder nur Begabungs- oder nur Anstrengungsfeedbacks erfolgten, der jeweils andere Ursachenfaktor im Sinne kompensierender Verknüpfungen in stärkerem Maße abgewertet wurde, als dies bei rein internen Attribuierungen der Schülerinnen und Schüler der Fall war. Die Tatsache, dass jemand einem gegenüber immer wieder betont, dass man begabt sei oder aber, dass man sich sehr anstrenge, scheint demnach die Tendenz eines einseitigen Attribuierungsstils zu verstärken.

Die vorliegenden Befunde implizieren [...], dass zwischen Selbstattribuierungen und der Verarbeitung von Fremdattributionen spezifische Unterschiede bestehen, die gerade daraus resultieren, dass Beobachter in der Regel nur die wichtigste von ihnen wahrgenommene Ursache mitteilen, während eigene Handlungen durch multiple Bedingungen erklärt werden. (Dresel, 2004, S. 205)

Bemerkenswerterweise zeigte sich in der Studie auch, dass sich bei der Darbietung von lediglich einem der beiden Ursachenfaktoren die oben angesprochenen negativen Effekte einstellten: Kontinuierliches Fähigkeitsfeedback führte bereits kurzfristig zu einer Anstrengungsreduktion und, vermittelt über subsequent eingetretene Misserfolge, längerfristig (Posttest nach zwei Wochen sowie 
Follow-up nach sechs Monaten) zu ungünstigen Kontroll- und Wertkognitionen sowie entsprechenden Emotionen. Die postulierten selbstwertgefährdenden Effekte von stetigen Anstrengungsfeedbacks manifestierten sich längerfristig in fehlenden Zuwächsen beim mathematikbezogenen Fähigkeitsselbstkonzept sowie in Abwertungen von mathematischen Aktivitäten. Schülerinnen und Schüler, die ausschließlich Fähigkeitsattributionen nach Erfolgen erhielten, gaben den Rückmeldungen ferner höheres Gewicht und bauten die Ursachenzuschreibung in stärkerem Maß in ihren Attributionsstil ein, als solche, die lediglich Anstrengungsattributionen erhielten. Dresel meint, dass dies dafür spreche, dass bei Fremdattributionen im Sinne eines self-enhancements (vgl. Abschnitte 5.3.2.2 und 5.5.1.4) vor allem die selbstwertdienlichen Ursachen in den eigenen Attributionsstil übernommen würden (vgl. Dresel, 2004, S. 205).

Attributionales Feedback nach Erfolgen, so lässt sich daraus schließen, sollte also sowohl aus fähigkeits- als auch aus anstrengungsbezogener Information bestehen. Die Sequenzierung der beiden Ursachenzuschreibungen dürfte dabei aber zentral sein. Bei einer intermittierenden Darbietung, also der zufälligen Fremdzuschreibung mal der einen, mal der anderen Ursache, ergeben sich gemäß der Studien von Dresel (2004) und Schunk (1983) keine vorteilhafteren Effekte als bei der ausschließlichen Darbietung lediglich eines der beiden Faktoren. Vielmehr gibt es Hinweise, dass es ratsam ist, eine Feedbackstrategie zu wählen, bei der Erfolge zeitlich separiert zu Beginn der Beschäftigung mit einem neuen Lernbereich erst mit Anstrengung und mit fortschreitendem Wissenserwerb schließlich mit Fähigkeit erklärt werden. Wie Dresel belegen kann, ist diese Sequenzierungsform derjenigen mit der umgekehrten Reihenfolge deutlich überlegen:

Die Sequenzierungsform, die mit der Darbietung von Anstrengungsattributionen einsetzte, war jener, bei der Erfolge zunächst mit Fähigkeitsattributionen kommentiert wurden, in der unmittelbaren und vor allem in der langanhaltenden Wirkung deutlich überlegen. Schüler(innen), denen nach frühen Erfolgen bei der Bearbeitung [der Mathematiklernsoftware] Erklärungen durch ihre hohen ·Anstrengungen nahegelegt wurden, nahmen diese Attribution im Mathematikunterricht nach Erfolg und Misserfolg in stärkerem Ausmaß vor, sahen Misserfolge stärker als Ausnahmefall an, verfügten über höhere Kontrollüberzeugungen und bewerteten mathematische Aktivitäten höher als Schüler(innen), deren Erfolge zu Beginn des Trainings auf ihre hohen Fähigkeiten zurückgeführt wurden. (Dresel, 2004, S. 202)

In der Studie von Dresel und Ziegler (2006) ließen sich mit der Feedbacksequenz «Anstrengung-Fähigkeit» - und wiederum nur mit dieser Kombination - unmittelbare und über sechs Monate stabil bleibende Förderwirkungen bezüglich des 
fachlichen Selbstkonzepts, der Kontrollüberzeugungen sowie bezüglich der Hilflosigkeit feststellen. Insbesondere gelang es mit dieser Feedbacksequenz aber auch, kurzfristig und langfristig signifikante Trainingseffekte im Bereich der impliziten Fähigkeitstheorie der jugendlichen Proband*innen zu erzielen: Die Fremdattribution von Anfangserfolgen auf Anstrengung und das Zurückführen des Meisterns der zunehmend komplexer werdenden Problemstellungen auf Fähigkeit scheint in den Augen der Lernenden demnach nicht nur eine hohe Plausibilität und Glaubwürdigkeit zu besitzen, sondern auch die Sicht zu fördern, wonach Fähigkeit etwas durch Anstrengung Modifizierbares sei.

Jemandem gegenüber Vertrauen in dessen Kompetenz auszudrücken und Zweifel und Kritik zurückzuhalten dürfte gerade für Personen von großer Bedeutung sein, die sich in einer schwierigen Lage wähnen. Entsprechende Befunde zusammenfassend meint Bandura (1997, S. 101), jemanden in einer solchen Situation zu ermutigen, sich ihm gegenüber verbal, gestisch und mimisch optimistisch auszudrücken («Ich bin sicher, dass du das kannst»), erleichtere es dem Adressaten, auf die eigenen Stärken zu fokussieren, Selbstzweifel zurückzudrängen und einen zusätzlichen Effort zu leisten.

\subsection{Unterschiedliche Kontrollkonstrukte: Implikationen für die Analyse elterlicher evaluativer Feedbacks gegenüber dem Kind}

In der vorliegenden Studie stehen Interventionen in Form elterlicher «Kontrollregulationen» bzw. «evaluativer Feedbacks» - mit Kluger und DeNisi (1996, S. 235) verstanden als «actions taken by an external agent to provide information regarding some aspect(s) of one's task performance» - im Fokus. Ausgelöst a) durch Leistungsergebnisse des Kindes (z. B. die Note in einer Mathematikprüfung zu Satzaufgaben oder die Anzahl gelöster Aufgaben im Rechenbuch etc.), b) durch Beobachtungen des Verhaltens des Kindes im Rahmen des häuslichen schulbezogenen Lernens (z. B. «Sie ist genervt und trödelt in ihrem Zimmer herum, statt auf die anstehende Englischprüfung zu lernen») oder c) durch Schilderungen des Kindes hinsichtlich seiner Emotionen und Handlungen im Kontext von Lern- und Leistungssituationen (z. B. «Mama, ich fürchte mich vor der anstehenden Prüfung») bzw. d) durch Schilderungen Dritter (z. B. «Deine Lehrerin meinte, du würdest dich im Unterricht zu wenig melden...») evaluieren die Eltern vor dem Hintergrund ihrer bildungsbezogenen Überzeugungen, kindspezifischen Erwartungen generellen und Aspirationen (vgl. Abschnitte 2.2.2.4 und 4.2.1.2) in den interessierenden Handlungsepisoden die Situation, indem sie ihrem Kind 
gegenüber einen bestimmten aus ihrer Sicht bedeutsamen Aspekt hervorheben und eine Aussage dazu machen, inwieweit es diesen Aspekt beherrsche bzw. unter Kontrolle oder «im Griff» (Frenzel \& Stephens, 2017, S. 38) habe.

Allerdings werden solcherlei kontrollbezogenen Aussagen sprachlich vielfältig realisiert, was vom Kind und den Forschenden Interpretationsleistungen abverlangt. Während in den betreffenden Sequenzen die Intention des Elternteils, dem Kind evaluatives Feedback zu geben, meist auf Anhieb zu erkennen ist, fällt es auf den ersten Blick nicht immer ebenso leicht zu entscheiden, auf welchen Aspekt der Elternteil in seinem Feedback genau abzielt. Dies lässt sich an der folgenden, bereits in Abschnitt 1.2 kurz erläuterten Sequenz der Mutter S11 illustrieren:

S11: [...] es nervt mich manchmal auch ein bisschen, wenn ich sage: «K03, wach auf!» [...] Ich sage: «K03, du bist fähig, Fünfen nach Hause zu bringen. Warum kommst du manchmal mit einer Viereinhalb oder Vier nach Hause? Nur weil du es ein bisschen locker genommen hast?» [...] Deswegen nervt es mich, wenn auch die Lehrerin und der Lehrer sagten: «Du kannst es, aber du willst es einfach nicht wahrhaben». (Interview G1, 00:40:52)

Die zweite eingeschobene direkte Rede (Ich sage: «K03, du bist fähig, ...) ist explizit kompetenz- bzw. kontrollbezogen und besteht aus mehreren Teilaussagen: «du bist fähig, Fünfen ${ }^{15}$ nach Hause zu bringen», «du kommst manchmal mit einer Viereinhalb oder einer Vier nach Hause» sowie «es liegt daran, dass du jeweils zu wenig ernsthaft lernst». Der Folgesatz macht deutlich, dass die beiden letzten Aussagen zwar als Fragen formuliert waren, es sich dabei aber um rhetorische Fragen handelt, mit denen die Mutter ihren Aussagen mehr Prägnanz verleihen möchte. Ferner gibt es in der Sequenz noch eine dritte direkte Rede, in der sich die Mutter auf Aussagen der Lehrkraft bezieht, welche sie inhaltlich teilt: «du kannst es (Fünfen schreiben)», «du schätzt die Bedeutung eines solchen Handelns unzureichend ein».

Worin besteht die kontrollbezogene Botschaft bzw. bestehen die kontrollbezogenen Botschaften, die der Elternteil dem Kind in dieser Episode vermitteln will? Gemäß der Systematisierung von Kontroll-Konstrukten Skinners (1996) gilt es, im Sinne der agent-ends relation (vgl. Abbildung 5.2) das Situationselement zu suchen, auf das der Elternteil hier mit seiner Aussage abzielt: Was ist es, was das Kind in den Blick nehmen soll? Worüber möchte der Elternteil dem Kind ein aus seiner Sicht angemessenes Bild dazu vermitteln, inwiefern es diesen Aspekt «im Griff» habe? Um dies zu analysieren, müssen das Kind und die Forschenden

${ }^{15}$ Die Note 5 entspricht in der Schweiz dem Prädikat «gut» (vgl. Fußnote 3). 
die Teilaussagen so in Beziehung setzen, dass eine stringente Botschaft erkennbar wird.

Diese könnte in einem ersten Schritt so lauten: «Merke dir, du bist fähig, gute Noten nach Hause zu bringen, aber manchmal bringst du nur mäßige oder knapp genügende Noten nach Hause, was daran liegt, dass du das Lernen zu locker nimmst». Ersetzt man die Wendung nach Hause bringen mit den situationsnaheren Verben hervorbringen oder schreiben, so entsteht daraus der Satz: «Du bist fähig, gute Noten zu schreiben, aber manchmal bringst du nur mäßige oder knapp genügende Ergebnisse hervor, was daran liegt, dass du das Lernen immer mal wieder zu locker nimmst».

Bei der ersten Teilaussage «Du bist fähig, gute Noten zu schreiben» äußert der Elternteil einerseits einen agent-means belief bzw. eine kompetenzbezogene Überzeugung, wobei es sich um eine globale selbstwirksamkeitsbezogene Aussage handelt, insofern als keine spezifische Domäne genannt wird und das eigentlich vorhandene Potential einer erfolgreichen Handlungsdurchführung thematisiert wird («du kannst deine Intentionen grundsätzlich erfolgreich umsetzen»).

Legt man den Fokus der Aussage stärker auf den Aspekt des genannten Ergebnisses («du kannst Fünfen schreiben»), so tritt die agent-ends-Dimension dieser Aussage stärker hervor, die im nächsten Teilsatz aber eingeschränkt wird («du bringst manchmal Viereinhalben und Vieren hervor». Der Aspekt, auf den die Mutter mit ihrer kontrollbezogenen Aussage mit hoher Wahrscheinlichkeit abzielt, aber nicht wörtlich so formuliert, dürfte hier in der Stabilität der Erbringung guter Leistungen liegen: «Du hast das Erbringen stabil guter Leistungen nicht im Griff».

Im nächsten Teilsatz wird ferner die Kausalitätsüberzeugung, also der meansends belief, aber indirekt auch ein weiterer agent-means belief der Mutter deutlich: «weil du es manchmal mit dem Lernen zu locker nimmst». Das Nichtim-Griff-haben der - eigentlich möglichen - Zielerreichung liegt mit anderen Worten am unsteten und unzureichenden Erarbeiten des betreffenden Wissens.

Formalisieren lassen sich die bisherigen Analyseschritte in folgendem Frame: «Merke dir: Du hast ... [den schulischen task X] [in bestimmten Maß (nicht)] im Griff, weil du ... [vom Elternteil vorgebrachte attributionale Argumente Y und Z].»

Im Rahmen des Codierens oblag es den analysierenden Personen, das vom jeweiligen Elternteil in der kontrollbezogenen Handlungsepisode Gemeinte möglichst nah an der Originaläußerung in der geschilderten Weise in die mit eckigen Klammern versehenen offenen Stellen dieses Frames einzufügen (vgl. Abschnitt 1.2 und Abschnitt 6.4.2). So wurden die sprachlich vielfältigen Stellen im Hinblick auf die Inhaltsanalyse in ein einheitliches Format gebracht, das die relevante Information bezüglich ihres propositionalen Gehaltes deutlich 
hervortreten ließ (vgl. Linke, Nussbaumer, Portmann, Willi \& Berchtold, 2004, S. 210-216). Für das vorliegende Beispiel resultiert bisher demnach die geframte Aussage:

«Merke dir: Du hast das Erbringen stabil guter Leistungen [in bestimmtem Maß] nicht im Griff, weil du dir das notwendige Wissen immer mal wieder nur unzureichend erarbeitest.»

\subsubsection{Die Darbietung attributionaler Argumente durch die Eltern: Ein Kategoriensystem}

Nun stellt sich aus der Sicht des Kindes (und der Forschenden) die Frage, wie sehr der Elternteil glaubt, dass es den betreffenden, offenbar wichtigen Lern- und Leistungsaspekt «im Griff» - bzw. wie im vorliegenden Beispiel - «nicht im Griff» habe. Für das Kind dürfte diese Information zentral sein, gibt sie ihm doch Hinweise dazu, wie zuversichtlich oder pessimistisch es bezüglich des Meisterns aktueller oder zukünftiger Lern- oder Leistungssituationen sein kann.

Im Kontext der Befunde zu den Effekten von Attributionen bzw. von attributionalem Feedback auf Kontrolleinschätzungen und -überzeugungen (vgl. Abschnitt 5.3.3.2) wird deutlich, dass hierfür die im Weil-Satz formulierten

Gründe bzw. deren konkrete Konfiguration hinsichtlich der Stabilitäts-, der Kontrollierbarkeits- und der Lokus-Dimension dem Kind (und den Forschenden) die entscheidenden Hinweise geben.

Dies lässt sich zuerst an zwei wenig komplexen Begründungstypen (vgl. Abbildung 5.3) illustrieren: So signalisiert der Elternteil, wie in Abschnitt 5.3.3 unter dem Stichwort situation-outcome expectancy angesprochen, beispielsweise eine hohe Kontrolle, wenn er mit Aussagen zur Situation operiert, die durch stabile Bedingungen gekennzeichnet sind und per se mit hoher Wahrscheinlichkeit ein für das Kind günstiges, also positiv-valentes Ergebnis zeitigen: z. B. «weil du eine Lehrerin hast, die dir immer gut gesinnt ist» bzw. «weil vor den Ferien keine Zeit mehr ist, eine Prüfung durchzuführen». Obwohl in diesem Fall die Kontrollierbarkeit, also die willentliche Beeinflussbarkeit durch das Kind, gering ist, signalisiert der Elternteil, wenn er mit einem Weil-Satz operiert, der positiv-valente stabile Bedingungen in der Situation oder bei Handlungspartnern anspricht, dass es die entsprechende schulische Aktivität oder das Leistungsereignis «gut im Griff habe» und folglich zuversichtlich sein könne. Unter der Bedingung, dass das Kind dem elterlichen evaluativen Feedback Glauben schenkt (vgl. Abschnitt 5.3.3.2 und 5.7) und den elterlichen Appell zur Internalisierung des Feedbacks («Merke dir!») 
ernst nimmt, erzeugen die Eltern mit dem Einsatz diese Begründungstyps die aktivierende Emotion Hoffnung (vgl. Pekrun, 2006, S. 320; Pekrun \& Perry, 2014, S. 121; Weiner, 1985, S. 566). Umgekehrt dürfte die signalisierte Kontrolle sehr tief sein («du hast es sehr schlecht im Griff»), wenn der Elternteil stabil ungünstige durch das Kind nicht willentlich beeinflussbare Bedingungen - im Sinne einer negativen situation-outcome expectancy - ins Feld führt: z. B. «weil dir in unserer Familie niemand bei diesen Mathematikhausaufgaben helfen kann». In diesem Fall wird dem Kind bedeutet, dass bezüglich einer aktuellen schulischen Aktivität oder eines anstehenden Leistungsereignisses kein Grund für Zuversicht bestehe. Falls das Kind das im Weil-Satz eingesetzte Argument für glaubwürdig bzw. zutreffend hält, dürften die Eltern bei ihm zumindest Angst erzeugen, und falls es für sich selber keine Argumente aufbringt, die seine (Netto-)Kontrolleinschätzung zu erhöhen vermögen (z. B. «ich könnte wohl morgen vor dem Unterricht mit meinen Freunden noch etwas auf die Mathematikprüfung lernen») gar die negativ deaktivierende Emotion Hoffnungslosigkeit evozieren (vgl. Pekrun, 2006, S. 320; Pekrun \& Perry, 2014, S. 121; Weiner, 1985, S. 566).

Wie hoch oder tief ist nun der signalisierte Kontrollgrad im Beispiel der oben erörterten Handlungsepisode der Mutter S11 zu veranschlagen? Die in den Frame übertragene Aussage lautet bisher: «Merke dir: Du hast das Erbringen stabil guter Leistungen [in bestimmtem Maß] nicht im Griff, weil du dir das notwendige Wissen immer mal wieder nur unzureichend erarbeitest.» Im Weil-Satz wird hier das «unzureichende» Handeln des Kindes angesprochen, was die im Hauptsatz signalisierte Kontrolle ins Negative wendet: «Du hast es nicht im Griff». Der Grad des «Nicht-im-Griff-Habens» bemisst sich nun an den Ausprägungen, die die drei Kausaldimensionen Lokus, Stabilität und Kontrollierbarkeit aufweisen: Das vom Elternteil hervorgebrachte negativ-valente Argument beruht auf einem internalen Aspekt («etwas Unzureichendes, das in dir liegt»), der aber willentlich beeinflussbar ist («dein unzureichendes, aber grundsätzlich veränderbares Lernen) und wenigstens nicht ständig, sondern lediglich variabel auftritt («dein unzureichendes manchmal auftretendes Lernen»). Vor allem der Umstand, dass der vorgebrachte negative Grund durch das Kind veränderbar ist und schon jetzt nicht ständig auftritt, dürfte dem Kind signalisieren, dass der Elternteil nicht der Meinung ist, dass Grund für Hoffnungslosigkeit bezüglich des in der Kontrollregulation thematisierten Zielaspekts - hier das Erbringen stabil guter Leistungen - bestehe, und mit anderen Worten zwar nur ein geringes Maß an «nicht-im-Griff-Haben» vorliege, aber dennoch Anlass bestehe, sich deswegen Sorgen zu machen:

«Merke dir: Du hast das Erbringen stabil guter Leistungen eher schlecht im Griff, weil du dir das notwendige Wissen immer mal wieder nur unzureichend erarbeitest.» 
Wie in den obigen drei Beispielen deutlich wird, wurde in der Analyse des Datenmaterials (vgl. Abschnitt 7.2.3.3) eine ordinalskalierte Codierung des vom Elternteil signalisierten Maßes an vorhandener Kontrolle ( $«$ du hast es im Griff) und nicht-vorhandener Kontrolle («du hast es nicht im Griff») gewählt und mit den wertenden Ausdrücken «gut» und «schlecht» ausgedrückt: «Du hast es eher gut - gut - sehr gut im Griff» bzw. «du hast es eher schlecht - schlecht - sehr schlecht im Griff» ${ }^{16}$.

Abbildung 5.3 illustriert das an Weiners Klassifikationsschema orientierte und an den Daten entwickelte Kategoriensystem zur Codierung der von den Eltern in ihren Kontrollregulationen angeführten Argumente. Entlang der vier Dimensionen Lokus, Stabilität, Kontrollierbarkeit sowie Valenz des Arguments mit jeweils zwei Ausprägungen bilden sich 16 Begründungstypen, welche die von der Attributionsforschung herausgearbeiteten Konsequenzen auf die Kontrollüberzeugungen und das emotionale Erleben der Kinder entfalten dürften (vgl. Abschnitt 5.3.3.1 und 5.3.3.2).

Die meisten Elternteile, die an der vorliegenden Studie teilnahmen, mögen zwar immer mal wieder den Eindruck gehabt haben, ihre Kinder hätten bezüglich spezifischer unterrichts- und übertrittsbezogener Aspekte unrealistisch hohe Kontrollüberzeugungen, und es sei nötig, diesbezüglich mittels entsprechender Feedbacks korrektiv Einfluss zu nehmen («Merke dir: Du hast das angemessene Einschätzen deiner Fähigkeiten in ... schlecht im Griff, weil du ...»). In Anbetracht dessen, dass die 20 Kinder aber nicht zuletzt wegen ihren bisherigen Fachleistungen ${ }^{17}$ in der Erhebungsphase mit einem unklaren Übertrittstatus konfrontiert waren und alle wenigstens zeitweise in erhöhtem Maß von Selbstzweifeln, Unsicherheit und Druck geplagt sein mochten, ist davon auszugehen, dass ein adaptives kontrollbezogenes Handeln der Eltern in dieser Phase generell darin bestand,

a) Aspekte, die ihren Zielvorstellungen bereits entsprachen ( $\ll$ du hast es im Griff»), gegenüber dem Kind affirmativ durch den Verweis auf internale und stabile Faktoren - sich laufend weiterentwickelnde Fähigkeiten, stetiger Einsatz produktiver Strategien, stetige Anstrengung und Aufmerksamkeit - zu verstärken, und

\footnotetext{
${ }^{16} \mathrm{Im}$ Ergebnisteil wird hierfür mitunter auch der Ausdruck «Zugeschriebene Kontrolle» mit den Wertelabels eher hoch - hoch - sehr hoch sowie eher tief - tief - sehr tief verwendet (vgl. Abschnitt 7.2.3.3)

${ }^{17}$ Die mittleren Zeugnisnoten der 20 Kinder lagen während der Erhebungsphase in Mathematik bei $\mathrm{M}=4.2(\mathrm{SD}=0.5)$ und in Deutsch bei $\mathrm{M}=4.4(\mathrm{SD}=0.2)(\mathrm{vgl}$. Abschnitt 4.3, Tabelle 4.4).
} 


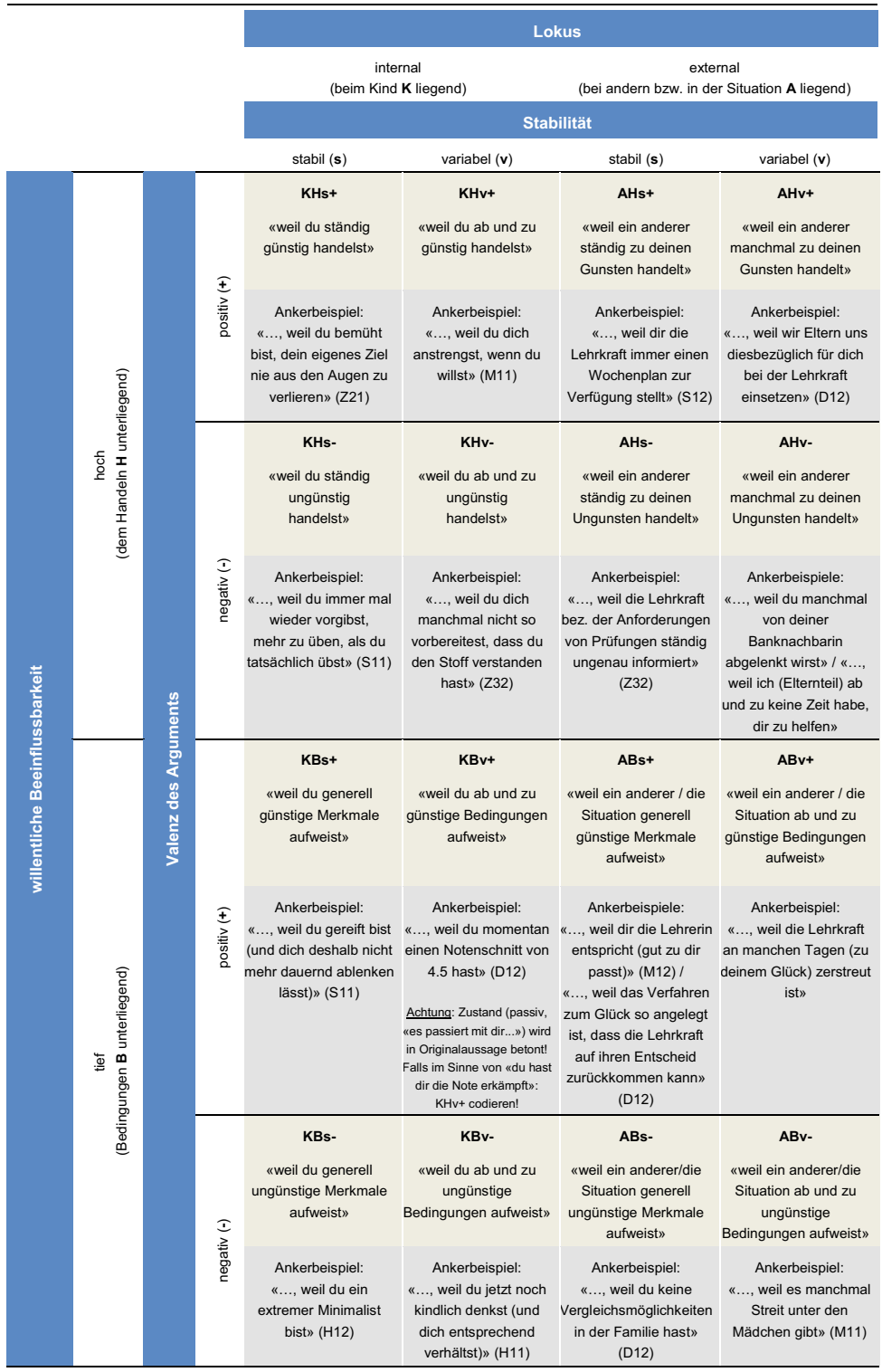

Abbildung 5.3 Kategoriensystem «Elterliche Strategien der verbalen Kontrollvermittlung» (sog. «kontrollbezogene Begründungstypen») 
b) Aspekte, die ihren Zielvorstellungen noch nicht entsprachen («du hast es nicht im Griff»), gegenüber dem Kind durch den Verweis auf kontrollierbare und variable Faktoren - vor allem auf sporadisch noch auftretendes ungünstiges eigenes Handeln - zuversichtlichkeitsstiftend, aber auch gesichtswahrend in produktive Bahnen zu lenken (vgl. Abschnitt 5.3.3.2).

Für die dazu notwendige Graduierung der in einem ersten Analyseschritt lediglich dichotom codierten Kontrollzuschreibungen («du hast es im Griff» vs. «du hast es nicht im Griff») wurde die in Tabelle 5.2 dargestellte Recodierungsmatrix geschaffen. Jedem der acht positiv-valenten und acht negativ-valenten Begründungstypen wurde gemäß den folgenden Überlegungen das in der dritten Spalte angegebene Gewicht hinsichtlich seines kontrollförderlichen Potentials beigemessen:

Bei positiv-valenten Kontrollaussagen ( $«$ du hast es mehr oder weniger gut im Griff») sind in erster Linie die Kausaldimensionen Lokus und Stabilität von hoher Relevanz für das Kind. Die Zuversicht, auch weiterhin erfolgreich zu sein, steigt umso mehr an, als sich dieses selbst als Urheber des Erfolgs (internal) erkennt und/oder sich ihm die erfolgsversprechenden Umstände als invariant darstellen.

Bei der Graduierung der Begründungstypen wurden demzufolge die Ausprägungen internal und stabil jeweils mit einem Punkt «positiv gewichtet» (vgl. Tabelle 5.2, dritte Spalte). Und aus den Summen der positiven Gewichte pro Begründungstyp resultierte schließlich die Zuordnung zu den drei Abstufungen «sehr gut im Griff», «gut im Griff» und «eher gut im Griff». Bei negativ-valenten Kontrollaussagen («du hast es mehr oder weniger schlecht im Griff») erweisen sich demgegenüber alle drei Kausaldimensionen von Bedeutung für den zugeschriebenen Grad an Kontrolle: Die Hoffnung, in absehbarer Zeit erfolgreich zu sein, bleibt umso geringer, je stärker das Kind äußere Faktoren (external) als Urheber des Misserfolgs sieht, es die negativen Faktoren als invariant (stabil) erachtet und es strukturelle Merkmale (unkontrollierbar) als verantwortlich für die ungünstige Situation zu erkennen glaubt. Entsprechend wurden bei der Graduierung der negativen Begründungstypen die Ausprägungen external, stabil und unkontrollierbar je mit einem Punkt «negativ gewichtet» (vgl. Tabelle 5.2, dritte Spalte). Analog zum Vorgehen bei den positiv-valenten Kontrollaussagen wurden die negativen Begründungstypen entlang ihrer summierten negativen Gewichte den Abstufungen «eher schlecht im Griff», «schlecht im Griff» und «sehr schlecht im Griff» zugeordnet.

Im abschließenden Abschnitt 5.7 wird der einleitende Teil des Frames «Merke dir!» im Zentrum stehen, der auf die appellative Funktion solcher evaluativen Feedbacks aufmerksam macht. Damit die Wahrscheinlichkeit steigt, dass das 


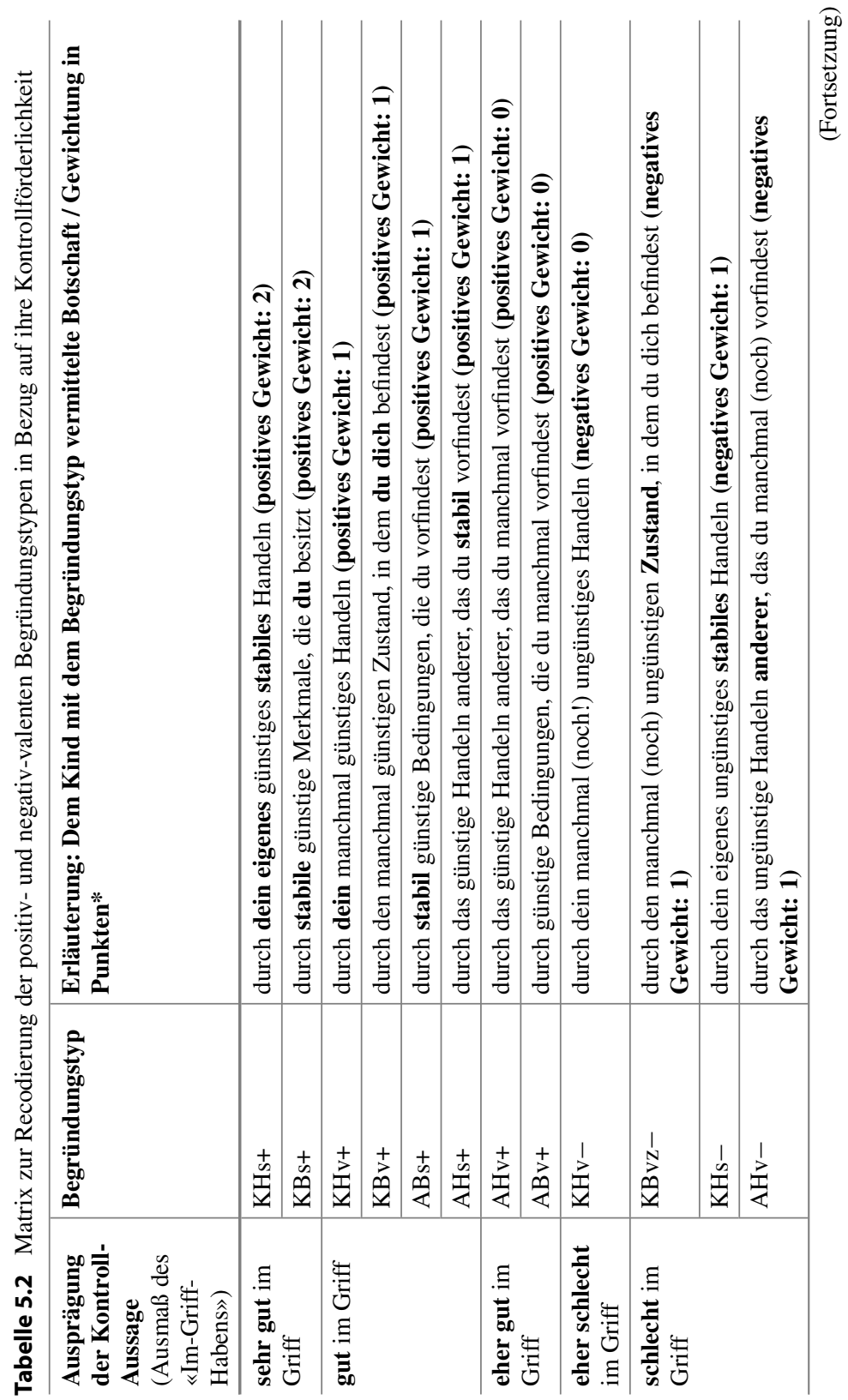




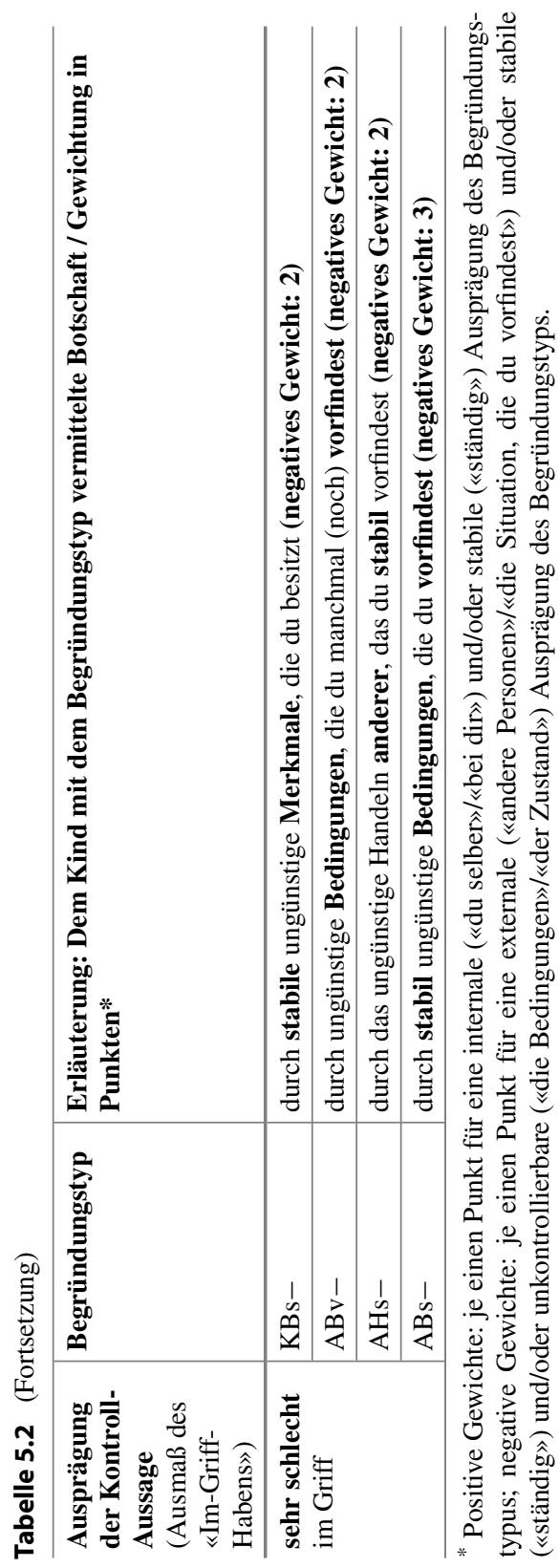


Kind die kontrollbezogene Botschaft der Eltern ernsthaft erwägt und nachhaltig in sein Überzeugungssystem integriert, dürfte nicht nur entscheidend sein, für wie akkurat, glaubwürdig und selbstwertdienlich es die elterlichen Argumente im Vergleich zu den Ergebnissen seiner eigenen kausalen Suche einschätzt (vgl. Abschnitt 5.3.3.2), sondern auch, welche Merkmale der Kommunikationsstil bzw. die Beziehungsqualität zwischen den Eltern und Kindern aufweist (vgl. auch Abschnitt 2.2.2.4).

Wie sich noch zeigen wird, dürfte letzteres in vermutlich noch höherem Maß für die Internalisation von elterlichen Wertzuschreibungen gelten. In den folgenden Kapiteln wird nun ein Licht auf die Wertkomponente geworfen und die Rolle erörtert, die verschiedene Facetten derselben gemäß erwartungswert-theoretischen Konzeptionen im Lern- und Leistungsgeschehen spielen. Wie bei den vorangehenden Kapiteln zur Kontrollkomponente leiten diese Erörterungen über zur Beschäftigung a) mit den Wirkungen, die verschiedene verbale Wertzuschreibungen der Eltern auf das schulbezogene Denken und Handeln ihrer Kinder entfalten, und b) mit der Gestaltung eines Frames, mit dem sich - analog zum skizzierten Vorgehen bei den Kontrollregulationen - die elterlichen Schilderungen eigener verbaler Wertzuschreibungen im Hinblick auf die nachfolgenden Analysen nach bestimmten, theoretisch relevanten Merkmalen vereinheitlichen lassen.

\subsection{Subjektive Werteinschätzungen}

«Wert» tritt in Atkinsons formalisiertem Risiko-Wahl-Modell (vgl. Abschnitt 5.1) als Anreizwert einer Leistungssituation (incentive value of success) in Erscheinung, der letztlich allein in der Schwierigkeit der Zielerreichung gründet: Je schwieriger die Aufgabe ist, desto reiz- bzw. wertvoller ist sie, insofern als damit im Kontext des Leistungsmotivs positiv-valente affektive Konsequenzen «pride in accomplishment» (Atkinson, 1957, S. 360) - antizipiert werden. Wird die Aufgabe aber vor dem Hintergrund vergangener Misserfolgserfahrungen mit ähnlichen Situationen als zu schwierig wahrgenommen, ist auch die Wahrscheinlichkeit des Scheiterns und damit das Erleben von negativ-valenten affektiven Zuständen (Scham) hoch, was es zu vermeiden gilt. Die beiden motivationalen Zustände drückt Atkinson (1957, S. 362) mathematisch aus, indem er den wahrgenommenen positiven Anreizwert $I_{S}$ als invers zur subjektiv eingeschätzten Erfolgswahrscheinlichkeit $\mathrm{P}_{\mathrm{S}}$ definiert (also: $\mathrm{I}_{\mathrm{S}}=1-\mathrm{P}_{\mathrm{S}}$ ) und den Anreizwert 
Misserfolg zu vermeiden, $\mathrm{I}_{\mathrm{f}}$, als negativ zur Erfolgswahrscheinlichkeit operationalisiert $\left(\mathrm{I}_{\mathrm{f}=-} \mathrm{P}_{\mathrm{S}}\right)^{18}$. Abgesehen davon, dass die Wertkomponente so lediglich im Kontext von affektiven, weitgehend unbewussten Motiven (achievement motive bzw. motive to avoid failure) gedacht wird, verschwindet sie mit dieser Operationalisierung über die Aufgabenschwierigkeit im Prinzip aus dem Blickfeld. Atkinsons einflussreiche Konzeption habe damit eine Konstellation geschaffen, so meinen Eccles und Wigfield (1995, S. 216), die vermutlich entscheidend dazu beigetragen habe, dass der Wert-Komponente in Theorie und Forschung weit weniger Aufmerksamkeit zugekommen sei als der Kontroll- bzw. Erwartungskomponente.

\subsubsection{Die Theorie des Subjective Task Value von Eccles, Wigfield et al. (1992, 2005)}

Namentlich mit Bezug zu Arbeiten von Rokeach (1973) und Feather (1988, 1992) stellt die Forschungsgruppe um Eccles und Wigfield dieser formalisierten Sicht einen Ansatz entgegen, der «Wert» nicht primär als genuin motivationales und lediglich auf den Schwierigkeitsgrad einer Aufgabe bezogenes, sondern als kognitives Konstrukt versteht - sie sprechen von Wert-Überzeugungen (value beliefs) -, das sich auf verschiedene Funktionalitäten einer Aufgabe bzw. einer Domäne bezieht (im Sinne von zugeschriebenen «Bedeutsamkeiten»), welche von den Lernenden sowohl auf der Basis ihrer impliziten motivationalen Orientierungen und Bedürfnissen als auch auf der Grundlage ihrer Ziele und selbstbezogenen Überzeugungen erkannt werden:

[...] task values are determined by characteristics of the task as well as broader needs and values of the individual. These broader needs and values serve as the primary antecedents of the value individuals have for specific tasks, and Eccles et al. proposed four major antecedents of children's achievement values for different activities: their self-schema and goals, the relative perceived cost or benefit of doing the activity compared to doing other activities, the previous affective experiences individuals have had with different activities, and the perceptions of the values of their parents, teachers, and peers. (Wigfield \& Eccles, 1992, S. 289)

\footnotetext{
${ }^{18}$ Ist die Erfolgswahrscheinlichkeit hoch (z. B. $\left.P_{s}=.90\right)$, so ist der positive Anreiz tief $\left(I_{s}=\right.$ .10). Ist die Erfolgswahrscheinlichkeit hoch (z. B. $\left.P_{s}=.90\right)$, so ist das Gefühl der Erniedrigung bei einem Misserfolg - also der negative Anreizwert bzw. die Kosten - auch hoch $\left(I_{f}=-.90\right)$ (vgl. Atkinson, 1957, S. 362). Wie Wigfield und Eccles (1992, S. 281) schreiben, bezeichnet der incentive value of success Atkinsons damit im Kern den difficulty value einer Aufgabe.
} 
Individuen, so die grundlegende Annahme, engagieren sich bei denjenigen Aufgaben, denen sie hohen Wert zuweisen, und vermeiden diejenigen, denen sie geringen Wert zuschreiben (Simpkins et al., 2015b; Wigfield \& Cambria, 2010). Eccles, Wigfield und Kolleg*innen (vgl. Eccles, 2005; Wigfield \& Eccles, 1992) haben vier unterschiedliche Bedeutsamkeiten vorgeschlagen und hinsichtlich ihrer Faktorstruktur validiert (vgl. Eccles \& Wigfield, 1995), die Lernende in zur Verfügung stehenden Mitteln und anvisierten Zielen erkennen und die sich dann im Zusammenspiel mit Erfolgserwartungen auf die Wahl der Aktivität, auf das Engagement und die Persistenz bei deren Bearbeitung sowie auf die Leistungen auswirken: intrinsic value, attainment value, utility value und cost.

\subsubsection{Intrinsic value: «Weil es mich interessiert und Spaß macht!»}

Als intrinsischen Wert bezeichnen Eccles et al. das Vergnügen (enjoyment), welches ein Individuum in der Beschäftigung mit einer Aktivität verbindet oder das Interesse (interest), das es einem mit der Aufgabe verbundenen Aspekt entgegenbringt. Die Enjoyment-Facette ist laut Eccles (2005, S. 111) vergleichbar mit dem Flow-Konstrukt (vgl. Csikszentmihalyi, 1988) bzw. demjenigen der intrinsischen Motivation in der Selbstbestimmungstheorie (vgl. Ryan \& Deci, 2002, 2016; Vansteenkiste et al., 2006). Als Flow wird der Zustand bezeichnet, den Individuen erleben, wenn sie sich einer intrinsisch motivierten Aktivität widmen. Kennzeichen dieser Erfahrung sind u. a. eine vollumfängliche Absorbierung mit der Tätigkeit und ein «loss of reflective self-consciousness» (vgl. Nakamura \& Csikszentmihalyi, 2009, S. 90). Die Bedeutung der Aktivität ergibt sich für die Handelnden aus dem Spaß, den diese ihnen bereitet. Intrinsische Motivation bezeichnet in der Selbstbestimmungstheorie denn auch «the doing of an activity for its inherent satisfactions rather than for some separable consequence» (Ryan \& Deci, 2000a, S. 56) - eine Handlungsbereitschaft, die insbesondere dann gegeben ist, wenn Lernende in der betreffenden Situation Möglichkeiten erkennen, sich als selbstbestimmt und kompetent zu erleben (vgl. Ryan \& Moller, 2017, S. 215) ${ }^{19}$. Die zweite Facette von intrinsic value reflektiert die Forschungsarbeiten um das Interessekonstrukt. Interesse bezeichnet in der Pädagogischen Psychologie «eine besondere, durch bestimmte Merkmale herausgehobene [affektive und kognitive] Beziehung einer Person zu einem Gegenstand»(Krapp, 2018, S. 287), wobei dieser Gegenstand im schulischen Kontext ein konkretes Objekt, ein bestimmter Inhalt, eine ganze Objektklasse, ein Fach oder eine spezifische Aktivität sein kann.

\footnotetext{
${ }^{19}$ Der Prototyp intrinsisch motivierten Handelns erkennt die Arbeitsgruppe um Deci und Ryan im spontanen und proaktiven Explorieren, das kleine Kinder in Bezug auf neue und herausfordernde Aspekte ihrer Umwelt realisieren (vgl. Ryan \& Deci, 2000b, S. 70).
} 
Solcherlei besondere Person-Objekt-Relationen lassen sich denn auch danach unterscheiden, ob sie ähnlich wie flow plötzlich zustandsartig als situationales Interesse (situational interest) auftreten (z. B. wenn man von bestimmten Merkmalen eines Musikstücks gefesselt wird), oder ob sie als individuelles Interesse (individual interest) sich langsam aus situationalem Interesse entwickelnd (vgl. Hidi \& Renninger, 2006), aber relativ dauerhaft im Sinne einer stabilen dispositionalen positiv-valenten Orientierung gegenüber bestimmten Domänen (z. B. die Songs von Nick Cave, Free Jazz etc.) in Erscheinung treten (vgl. Krapp, Hidi \& Renninger, 1992; Schiefele, 2009). Die konzeptionelle Ähnlichkeit zwischen den Konstrukten situational interest und intrinsic value besteht namentlich darin, dass sich die situative kognitive und affektive Beziehung direkt auf Objekte (Gegenstände und Aktivitäten) richtet und nicht auf die Funktion, die diesen Objekten in Relation zu anderen Objekten oder Ereignissen zukommt (vgl. Eccles, 2005, S. 111; Schunk et al., 2014, S. 53). Im Zentrum der Aufmerksamkeit der Lernenden stehen die Mittel (means) (vgl. Abbildung 5.2), deren Ergründen, Verstehen und Beherrschen selbst zum Ziel wird: z. B. «I enjoy puzzling over mathematics problems», «When I'm working on a mathematics problem, I sometimes don't notice time passing», «If I can learn something new in mathematics/English, I'm prepared to use my free time to do so» (Trautwein et al., 2012, S. 777). Analytisches, relationierendes Nachdenken darüber, warum man sich mit einer Sache beschäftigen soll, ist somit zweitrangig. Der intrinsic value, den ein Individuum in einer Aufgabe erkenne, so Eccles (2005), beruhe vor allem auf seinen dispositionalen Persönlichkeitsmerkmalen und dem affektiven Erinnerungen prägender Erfahrungen mit denselben in der Vergangenheit:

The attraction to, or enjoyment of, particular types of activities are undoubtedly linked to core aspects of the self, such as temperament, personality, motivational orientations. It is also likely to be linked to both genetic propensities and to classical learning associated with either positive or negative emotional experiences during initial encounters with particular activities. (Eccles, 2005, S. 111)

Intrinsic value scheint zumindest in der Primar- und Sekundarstufe I vor allem ein wichtiger Prädiktor für Aktivitäten zu sein, bei denen eine freie Auswahl besteht, etwa für die Kurs- oder Fächerentscheidung sowie für die Wahl von Freizeitaktivitäten (z. B. Durik et al., 2006). Sodann gibt es vor allem aus Laborstudien zur Textverarbeitung Hinweise, dass das Einschätzen eines Textes als interessant (situationales Interesse) mit erhöhter Aufmerksamkeit und Konzentration, produktiverer Informationsverarbeitung sowie besseren Behaltensleistungen einhergeht (vgl. Hulleman, Godes, Hendricks \& Harackiewicz, 2010, S. 250; 
Krapp, 2018, S. 289). Ebenso zeigt sich in einzelnen Studien, dass erhöhtes situationales Interesse und enjoyment mit höherer Persistenz, z. B. beim Lesen anspruchsvoller Texte, assoziiert sind (vgl. Fulmer \& Tulis, 2013). In etlichen (nicht-experimentellen) Studien wird aber deutlich, dass der intrinsic value (eine Aufgabe interessant finden), anders als der utility value (eine Aufgabe als nützlich zur Erlangung zukünftiger Ziele erkennen), sich meist nicht als relevanter Prädiktor für Leistungsergebnisse erweist (zsf. Hulleman et al., 2016; Johnson \& Sinatra, 2013). Wie Cole, Bergin und Whittaker (2008) beispielsweise in einer Studie mit 1005 amerikanischen College-Studierenden zur Vorbereitung auf low stakes tests belegen, vermochte das allein Interesse, in den Mathematik-, Englisch-, Naturwissenschafts- und Sozialwissenschaftstests gut abzuschneiden, die Testleistungen im Gegensatz zur perzeptierten Wichtigkeit (attainment value) und zur perzeptierten Nützlichkeit eines guten Abschneidens (utility value) nicht zu prädiktieren: «interest to do well on the exam without appropriate levels of importance to do well is not conducive to test performance» (Cole et al., 2008, S. 621).

\subsubsection{Attainment value: «Weil ich es für mich als wichtig erachte!»} Mit attainment value - in Deutsch oft als Relevanz oder Wichtigkeit bezeichnet - verbinden Eccles et al. den Wert, den eine Aktivität für das Individuum hat, «because engaging in it is consistent with one's self-image» (Eccles, 2005, S. 109). Der Wert des Meisterns einer Aufgabe liegt in diesem Fall darin, dass diese bestimmte Elemente aufweist, in denen Lernende Gelegenheiten erkennen, vor sich und anderen bestimmte zentrale Aspekte ihres Selbst unter Beweis zu stellen oder zu widerlegen (Eccles \& Wigfield, 2002, S. 119). Je zentraler der betreffende Aspekt für die persönliche oder soziale Identität sei, so Eccles et al., desto wichtiger dürfte dem Individuum die Aktivität erscheinen (vgl. Eccles, 2005, S. 109). Anders als bei intrinsic value, steht hier die means-ends-Relation im Fokus: Die Aktivität erscheint dem Individuum als Mittel zum Zweck, allerdings handelt es sich bei letzterem nicht um ein vergleichsweise marginales Ziel wie beim utility value (vgl. Abschnitt 5.5.1.3), sondern um eines, das von ihm mit seinen grundlegenden Bedürfnissen und Wert-Überzeugungen in Verbindung gebracht wird - Aspekte also, die es als identitätsstiftend wahrnimmt: z. B. «Mathematics is important to me personally», «It is important to me personally to be a good mathematician» (Trautwein et al., 2012, S. 777). Konzeptionell schließen Eccles et al. hierbei namentlich an den Arbeiten von Rokeach (1973) und Feather (1988, 1992) zu Wert-Systemen an.

Rokeach (1979) definiert Werte als «core conceptions of the desirable within every individual and society», als proskriptive Überzeugungen (vgl. 
Abschnitt 4.1.1), die als Kriterien dienen, «to guide not only action but also judgment, choice, attitude, evaluation, argument, exhortation, rationalization, and, one might add, attribution of causality» (S. 2). Rokeach (1973, S. 7) unterscheidet zwischen terminalen Werten (terminal values) - es handelt sich dabei um Überzeugungen über die ultimativen menschlichen Lebensziele («desirable end states of existence») - und instrumentellen Werten (instrumental values), den erstrebenswerten Verhaltensweisen («modes of conduct») zur Erreichung der terminalen Werte. Während $\mathrm{zu}$ den terminalen Werten selbst- oder gesellschaftsbezogene Aspekte wie Weisheit, Freiheit, Selbstachtung oder soziale Anerkennung gehören, umfassen instrumentelle Werte moral- oder kompetenzbezogene Verhaltensmodi wie Ehrlichkeit, Verantwortlichkeit, Fähigkeit oder Intellektualität ${ }^{20}$. Laut Rokeach bilden diese im Rahmen der Sozialisation erlernten breiten Wertüberzeugungen in Form von persönlichen Wertsystemen - «hierarchical organizations along a continuum of importance» (Rokeach, 1973, S. 25) - in jeglichen Lebenssituationen relativ stabile Standards für die in ihnen stattfindenden kognitiven, affektiven und behavioralen Prozesse. Instrumentelle Werte, so Rokeach (1973, S. 14) wirkten hierbei motivierend, weil das Individuum sie als Mittel zur Erreichung der erwünschten Endziele erkenne. Terminale Werte seien ihrerseits motivierend, «because they represent the super goals beyond immediate, biologically urgent goals» (Rokeach, 1973, S. 14). Sie bildeten Ziele, die wir zwar dauernd erstrebten, aber im Gegensatz zu instrumentellen Werten nie ganz erreichten: «[...] we seem doomed to strive for these ultimate goals without quite ever reaching them» (Rokeach, 1973, S. 14).

Feather (1988, 1992) schloss bei der Konzeption seines Erwartungs-WertModells der Leistungsmotivation an Rokeachs Überlegungen zu instrumentellen

\footnotetext{
${ }^{20}$ Das von Rokeach entwickelte Messinstrument The Value Survey (vgl. Rokeach, 1973) erfasst je 18 Items für die beiden Wert-Kategorien. Terminale Werte: a comfortable life, an exciting life, a sense of accomplishment, a world at peace, a world of beauty, equality, family security, freedom, happiness, inner harmony, mature love, national security, pleasure, salvation, self-respect, social recognition, true friendship, wisdom. Instrumentelle Werte: ambitious, broadminded, capable, cheerful, clean, courageous, forgiving, helpful, honest, imaginative, independent, intellectual, logical, loving, obedient, polite, responsible, selfcontrolled (Rokeach, 1973, S. 28). Die Kritik an dieser Liste von Zielen und Verhaltensmodi bezieht sich vor allem auf deren Theorielosigkeit: Die genannten Wert-Ausdrücke stehen unrelationiert nebeneinander (vgl. Rohan, 2000, S. 260). Eine eigentliche Theorie über die Struktur des Wertsystems hat Schwartz (1992) vorgelegt: Hier positionieren sich die zehn Wert-Typen («motivational types of values») self-direction, stimulation, hedonism, achievement, power, security, conformity, tradition, spirituality, benevolence und universalism (Schwartz, 1992, S. 4-7) entlang der beiden bipolaren Dimensionen openness to change - conservation und self-enhancement-self-transcendence, zwei laut Schwartz (1992, S. 44) grundlegenden Problemen, die der Mensch lösen müsse.
} 
Werten an, indem er postulierte, dass diese maßgeblich determinierten, welchen Wert Individuen verschiedenen Aufgaben zumessen würden. Im Zusammenspiel mit der Erfolgserwartung, so meint er mit Blick auf Atkinsons Arbeiten, würden diese «task values» die Wahl, die Persistenz und das Ergebnis in Leistungssituationen prädiktieren. In einer Untersuchung zu den Studiengangsentscheidungen (enrollment decisions) von 444 angehenden Universitätsstudierenden konnte er zeigen (vgl. Feather, 1988), dass ein hoher task value und hohe Erfolgserwartungen für Mathematik mit der Entscheidung für ein naturwissenschaftliches Fach assoziiert waren und ein hoher task value und hohe Erfolgserwartungen für Englisch mit einer Entscheidung für ein geistes- oder sozialwissenschaftliches Fach einhergingen, wobei die subjektiven Werte und Erfolgserwartungen für die beiden Fächer anders als von Atkinson postuliert jeweils positiv korreliert waren (vgl. Abschnitt 5.1). Ferner zeigte sich, dass die task values für die beiden Fächer mit drei unterschiedlichen Wert-Profilen einhergingen, die Feather aus den instrumentellen Werten Rokeachs faktorenanalytisch gebildet hatte: Das WertProfil restrictive control (hohe Relevanz-Ratings für die instrumentellen Werte clean, obedient, polite, responsible und self-controlled) ebenso wie das WertProfil intellectual orientation (hohe Relevanz-Ratings für intellectual und logical) erwiesen sich als starke Prädiktoren für hohe task values bezüglich Mathematik und das Wert-Profil prosocial concern (hohe Relevanz-Ratings für forgiving, helpful und loving) für hohe task values bezüglich Englisch. In Pfadanalysen zeigte sich sodann durchgängig, dass die persönlichen Wert-Profile die Kurswahl nicht direkt, sondern stets vermittelt über die task values beeinflusst hatten. Die breiten, generalisierten instrumentellen Werte Rokeachs scheinen demnach vor allem auf die Bewertung von Situationen bzw. Aufgaben einen Einfluss zu entfalten, nicht aber auf das konkrete Handeln selbst (zumindest nicht auf das Wahlverhalten): Schülerinnen und Schüler zum Beispiel, die konsistentes, reflektiertes und rationales Vorgehen (mode of behavior bei den beiden instrumentellen Werten intellectual und logical) als ihren persönlichen Wesenszug erachten, erkennen offenbar in mathematikbezogenen Situationen und Aufgaben - anders als in sprach-bezogenen - einen höheren Wert, und erst Letzteres scheint sie entsprechend aufsuchend handeln zu lassen (vgl. Eccles, 2005, S. 110). Die «extreme domain specificity», die Marsh et al. (2012, S. 427) bezüglich der persönlichen Kompetenzzuschreibung konstatierten, scheint sich auch hinsichtlich der Wertzuschreibung zu bestätigen (vgl. Abschnitt 5.3.2.2).

Eccles, Wigfield und Kolleginnen orientieren sich bei der Konzeption des attainment value aber nicht nur an Arbeiten, die auf persönlichen Werten und Zielen beruhen, sondern verweisen auch auf konzeptionelle Ähnlichkeiten zu selbstbestimmt-extrinsischen Motivationsformen der Selbstbestimmungstheorie, 
die mit (sekundären) Bedürfnissen operiert. Namentlich das im Rahmen der Organismic Integration Theory (vgl. Deci \& Ryan, 1985; Ryan \& Deci, 2002, 2016) postulierte Konstrukt der integriert regulierten Motivation (integrated regulation) weist konzeptionelle Ähnlichkeit zu demjenigen des attainment value auf. Eine integrierte extrinsische Motivation liegt laut der Selbstbestimmungstheorie dann vor, wenn der Wert zwar nicht in der Handlung selbst, sondern in den Folgen liegt, aber die diesen Folgen zugeschriebenen Werte, welche ursprünglich in äußerer Regulation vermittelt wurden, in der Zwischenzeit vom Individuum so sehr ins eigene Selbst internalisiert worden sind, dass sie als selbstbestimmt empfunden werden: «It results when identifications have been evaluated and brought into congruence with the personally endorsed values, goals, and needs that are already part of the self» (Ryan \& Deci, 2002, S. 18). Obwohl solcherlei Handlungen einen instrumentellen Charakter hätten, so würden sie in ähnlich hoher Qualität ausgeführt wie intrinsisch motivierte, meinen Ryan und Deci (2000b, S. 62), da ihnen ein hoher Grad an Willentlichkeit bzw. internale Verursachung (internal locus of causality) zugrunde liege und damit dem Bedürfnis nach Autonomieerleben entsprochen werde (vgl. Abschnitt 5.5.1.1).

Dem Konstrukt attainment value, das demnach eine Vielzahl von unterschiedlichen persönlichen Bedürfnissen und Werten mit Bezug zu ihrer gemeinsamen Relevanz für die Bestätigung des Selbstbildes integriert (vgl. Barron \& Hulleman, 2015, S. 504), unterliegen zusammengefasst folgende Annahmen:

Individuals seek to confirm their possession of those characteristics central to their self-image; (2) various tasks provide differential opportunities for such confirmation; (3) individuals place more value on those tasks that either provide the opportunity to fulfill their self-image or are consistent with their self-image and long-range goals; and (4) individuals are more likely to select tasks with high subjective value than tasks with lower subjective value. (Eccles, 2005, S. 111)

Eccles, Wigfield und Kolleginnen sehen diese Zusammenhänge namentlich auch bezogen auf die geschlechtliche Identität von Menschen bestätigt. Aktivitäten und Aufgaben, die dem eigenen Bild von Maskulinität und Femininität widersprechen, dürften einen tiefen, solche, die ihm entsprechen, einen hohen attainment value aufweisen. Wie die Forschungsgruppe verschiedentlich zeigen konnte (zusf. Simpkins et al., 2015b, S. 20-21), werden schulische Domänen, aber auch Freizeitaktivitäten entlang von Geschlechtsnormen und -stereotypen bewertet. Über verschiedene Altersgruppen hinweg zeigt sich insbesondere bezüglich Sport und Lesen ein konsistenter Unterschied im attainment value zwischen den Geschlechtern: Während Jungen sportlichen Aktivitäten generell höhere Relevanz zuweisen 
als Mädchen, ist es beim häuslichen Lesen sowie in sprachlichen Fächern umgekehrt (z. B. Andre, Whigham, Hendrickson \& Chambers, 1999; Fredricks \& Eccles, 2002; Jacobs et al., 2002; Wigfield et al., 1997). Weniger eindeutig ist die Befundlage bezüglich der persönlichen Relevanz, die dem Fach Mathematik zugewiesen wird. Während in einigen Studien der Wert von Mathematik von Jungen höher veranschlagt wird als von Mädchen (z. B. Andre et al., 1999), zeigen sich anders als beim mathematikbezogenen Fähigkeitsselbstkonzept in etlichen Studien keine statistisch relevanten Unterschiede (vgl. Fredricks \& Eccles, 2002; Jacobs et al., 2002). Generell erweist sich der einer Aufgabe zugeschriebene attainment value nicht nur für die Wahl von Bildungsgängen, sondern auch für das Engagement und die Persistenz bei der Aufgabenbearbeitung sowie (subsequent) für Testleistungen als prädiktiv (z. B. Battle, A. \& Wigfield, 2003; Cole et al., 2008; Johnson \& Sinatra, 2013; Simons, Dewitte \& Lens, 2003; Wigfield et al., 1997).

\subsubsection{Utility value: «Weil es nützlich ist»}

Utility value - die Zweckmäßigkeit oder Instrumentalität - bezeichnet den Wert, den ein Individuum einer Aufgabe zur Realisierung längerfristiger Ziele («facilitating one's long-range goals») bzw. zur Erreichung kurz- oder langfristiger externaler Belohnungen («helping [...] obtain immediate or long-range external rewards») zuweist (Eccles, 2005, S. 109). Im Fokus der Gedanken des Individuums steht somit das Ziel und weniger die Aktivität, die klar als Mittel zum Zweck wahrgenommen wird: z. B. «I'll need good mathematics/English skills for my later life (training, studies, work)», «Good grades in mathematics can be of great value to me later» (Trautwein et al., 2012, S. 777). Bei den Zielen kann es sich durchaus um Aspekte handeln, welche das Individuum als persönlich sehr bedeutsam erachtet wie z. B. Karriereziele. Eccles (2005, S. 112) schreibt, dass utility value inhaltlich denn auch der Konzeption der extrinsischen Motivation nahekomme, wie sie von der Selbstbestimmungstheorie konzipiert wird (vgl. Deci \& Ryan, 1985; Ryan \& Deci, 2002, 2016). Neben der oben angesprochenen integriert regulierten Motivation beschreibt diese auf dem Kontinuum zwischen Amotivation und intrinsischer Motivation noch drei weitere Formen der extrinsischen Motivation, die sich jeweils nach dem Grad der Internalisierung äußerer Regulation und folglich nach dem Grad wahrgenommener Autonomie oder Selbstbestimmung bei der Handlungsdurchführung unterscheiden. Identifiziert regulierte Motivation (identified regulation) stellt einen Handlungsantrieb dar, der auf einer bewussten persönlichen Akzeptanz oder Anerkennung des Handlungsziels beruht, auch wenn dieses (noch) nicht voll den eigenen Werten und Bedürfnissen entspricht: «When [people] identif[y] with an action or the value it expresses, they, at least at a conscious level, are personally endorsing it [...]» 
(Ryan \& Deci, 2002, S. 17). Ein Beispiel einer von Identifikation getragenen Aktivität wäre das Auswendiglernen einer Buchstabenliste durch ein Kind, weil es dies als relevant für sein eigentliches Ziel erachtet, das Schreiben zu erlernen (vgl. Ryan \& Deci, 2000a, S. 62). Introjeziert regulierte Motivation (introjected regulation) ist demgegenüber eine Handlungsbereitschaft, die auf Werten beruht, welche vom Individuum zwar ebenfalls internalisiert, aber (vorderhand) in noch geringerem Maß ins Selbst integriert wurden: Der Ort der Verursachung (locus of causality), so Ryan und Deci (2002, S. 17), werde dabei vom Individuum als eher fremdbestimmt bzw. «quite controlling» wahrgenommen, insofern als diese der Selbstwertsicherung und der Erzielung von Stolz bzw. Anerkennung dienlichen Aktivitäten von andern ausgeführt würden. Der höchste Grad an Fremdregulation und ein damit einhergehender Eindruck, von außen kontrolliert zu werden (external locus of causality), herrscht bei Handlungen vor, die das Individuum allein zur Erfüllung äußerer Anforderungen oder zur Erzielung einer Belohnung bzw. Verhinderung einer Strafe ausführt. Solcherlei external regulierte Motivation (external regulation) «is the only kind of motivation recognized by operant theorists $[\ldots]$, and it is this type of extrinsic motivation that was typically contrasted with intrinsic motivation in early lab studies and discussions» (Ryan \& Deci, 2000a, S. 62).

Anders als intrinsic value scheint die Zuschreibung von utility value nicht nur für die (freie) Wahl von Freizeitaktivitäten und von Fächern und Kursen (vgl. Bong 2001; Eccles \& Harold, 1991; Harackiewicz et al., 2008; Simons et al., 2003; Updegraff et al., 1996), sondern auch für Leistungsergebnisse prädiktiv zu sein (Bong 2001; Cole et al., 2008; Durik et al., 2006). Wie dies die Selbstbestimmungstheorie postuliert, dürfte nicht jede Facette von utility value gleichermaßen effektiv zu sein: So konnten z. B. Simons et al. (2003) in einer sportbezogenen Interventionsstudie mit 660 belgischen Schülerinnen und Schülern auf der Sekundarstufe II zeigen, dass wenn diesen vor dem Training einer Basketballsequenz die Zweckmäßigkeit der Aufgabe für ihr zukünftiges Spiel deutlich gemacht wurde («practicing this skill is useful because you will use this and similar skills in the future. The tips you will receive can be personally beneficial», S. 151) - ihnen also das Warum der Prozedur mit Blick auf zukünftige als persönlich relevant erachtete Situationen klar wurde -, sie dann bei der Aufgabenbearbeitung eher eine Mastery- als eine Performance-Orientierung aufwiesen, von mehr Spaß und Interesse berichteten, engagierter und persistenter trainierten und bessere Leistungsergebnisse erzielten, als wenn sie dies lediglich zur Erlangung einer unmittelbaren Belohnung tun sollten («You will practice this skill only today and you will not repeat the practice this year. Nevertheless, you have to practice because you will be tested at the end of the session.», S. 151) oder ihnen vorab 
zwar eine allgemeine persönliche Wichtigkeit, aber kein klarer praktischer Nutzen signalisiert wurde («Practicing is not directly useful for your educational training, but the tips you will receive can be personally beneficial.», S. 151). Ähnlich positive Befunde bei Persistenz- und Leistungsmaßen zeigten sich bei klar kommunizierter zukünftiger praktischer Zweckmäßigkeit in einer Interventionsstudie in einem Ausbildungsgang für 184 angehende Krankenpfleger*innen (Simons, Dewitte \& Lens, 2004). Bong (2001) konnte in einer längsschnittlichen Studie mit 168 koreanischen College-Studentinnen zeigen, dass die wahrgenommene Zweckmäßigkeit eines Bildungsganges die Selbstwirksamkeit der Teilnehmerinnen und subsequent deren Prüfungsleistungen prädiktierte. In Studien von Malka und Covington (2005) sowie Hulleman et al. (2010) mit Psychologieund Mathematikstudierenden an der Universität prädiktierte die wahrgenommene Instrumentalität von Studieninhalten für die zukünftigen beruflichen Ziele das Engagement während des Unterrichts bzw. die Leistungsergebnisse. Ebenso gibt es Hinweise, dass sich Kinder und Jugendliche auf der Sekundarstufe I hinsichtlich ihrer Berufswahl, anders als bei der Wahl von Freizeitaktivitäten, weniger davon leiten lassen, was ihnen Spaß macht (intrinsic value), sondern stärker davon, als wie nützlich ihnen ein bestimmter beruflicher oder schulischer Bildungsgang für ihre längerfristigen Karriere- und Lebensziele erscheint. So wurden in einer Längsschnittstudie von Durik et al. (2006) die Berufsaspirationen von 600 Schülerinnen und Schülern lediglich vom wahrgenommenen utility value, nicht aber vom intrinsic value prädiktiert. Zusammengefasst deuten diese Befunde an, dass utility value, besonders in der Facette «erkannte praktische ZweckmäBigkeit für zukünftige als persönlich wichtig anerkannte Aktivitäten und Ziele» unmittelbar zu einem erhöhten Anregungsgrad, größerem Engagement und einer gesteigerten Beharrlichkeit bei sich einstellenden Schwierigkeiten führt und subsequent in besseren Leistungen resultiert (vgl. Greene, Miller, Crowson, Duke \& Akey, 2004, S. 476; Hulleman et al., 2016, S. 252).

Fasst man die Befunde der Studien zusammen, welche die bisher erörterten Komponenten intrinsic value, attainment value und utility value nicht - wie das oft der Fall ist - zu einem gemeinsamen task value-Konstrukt zusammengeführt ${ }^{21}$, sondern als Einzelkonstrukte ausgewertet haben, und lässt man den Einfluss der expectancy-Komponente beiseite (für Befunde unter Einbezug derselben, s. weiter unten), so ergibt sich folgendes Gesamtbild bezüglich deren Einflüsse auf das Lern- und Leistungshandeln:

\footnotetext{
${ }^{21} \mathrm{Da}$ die drei Komponenten in etlichen Studien relativ hohe Interkorrelationen aufweisen (z. B. Trautwein et al., 2012), werden sie häufig zu einer gemeinsamen task value-Skala zusammengeführt (z. B. Anderman et al., 2001; Eccles, Wigfield, Harold \& Blumenfeld, 1993; Jacobs et al., 2002).
} 
- Der von einem Individuum perzipierte intrinsic value ist primär ein starker Prädiktor für dessen (freie) Wahl von Kursen- und Studienfächern sowie von Freizeitaktivitäten.

- Die perzipierte persönliche Wichtigkeit (attainment value) vermag das Wahlverhalten eines Individuums, aber auch das kognitive Engagement, die Persistenz und die Leistungsergebnisse zu prädiktieren.

- Die in einer Aufgabe erkannte Zweckmäßigkeit (utility value) vermag zwar auch das Wahlverhalten eines Individuums vorauszusagen, scheint aber noch stärker als der perzipierte attainment value ein Prädiktor für dessen kognitives Engagement und dessen Beharrlichkeit bei der Aufgabenbearbeitung sowie vermittelt darüber - für seine erzielten Leistungsergebnisse zu sein.

Die differentielle Voraussagekraft der drei positiv-valenten task valueKomponenten hinsichtlich des Leistungshandelns und der Leistungsergebnisse erklären sich Johnson und Sinatra (2013) folgendermaßen:

For achievement related tasks that stress students' performance, an attainment value
may be predictive of achievement because it may focus students on their task per-
formance as a whole. Utility values on the other hand, focus students' interaction on
those aspects of the task that are perceived as useful; and an intrinsic value focus a
student on aspects of the task that are interesting to them but may or may not have
direct relevance to performing well on the task itself. (Johnson \& Sinatra, 2013, S. 52)

Mit anderen Worten scheinen sich Lernende je nach wahrgenommenem task value unterschiedlich in einer Aufgabe zu engagieren: Erkennen sie eine Aufgabe mit Blick auf ihr Selbstverständnis als wichtig, so erledigen sie diese gemäß ihres Selbstanspruchs in der für sie üblichen Qualität («es gehört sich für mich so») und haben dabei die Aktivität als Ganzes im Auge. Erkennen sie dahingegen eine praktische Zweckmäßigkeit einzelner Aspekte der Aktivität mit Blick auf zukünftige Lern- und Leistungssituationen, so scheint dies zumindest kurzfristig ein erhöhtes Engagement und einen zusätzlichen Effort zur Meisterung dieser zielführenden Aspekte auszulösen («es lohnt sich, diesen Aspekt gut zu beherrschen»). Steht der intrinsic value im Vordergrund, so beschäftigt man sich dahingegen genau mit denjenigen Aspekten, die spontan als interessant und vergnüglich erscheinen. Das Beherrschen der gesamten Aktivität steht dabei aber nicht zwingend im Fokus. 


\subsubsection{Perceived Cost: «Weil es mit Mühen, Hindernissen und/oder negativen Gefühlen verbunden ist»}

Während die Formen extrinsischer Motivation der Selbstbestimmungstheorie immer eine Annäherungs- und eine Vermeidungskomponente aufweisen (externale Regulation bedeutet z. B., dass man eine Handlung entweder zur Erreichung einer Belohnung oder zur Verhinderung einer Bestrafung ausführt), ist utility value in der Konzeption von Eccles und Kolleginnen ebenso wie attainment value und intrinsic value stets mit einem positiven Anreiz verbunden - «the perceived [...] benefit of doing the task» (Wigfield \& Eccles, 1992, S. 289). Negative Merkmale, die eine Aktivität in den Augen des Individuums aufweist, fassen sie unter subjektive Kosten (perceived cost) zusammen:

The first three components are best thought of as attracting characteristics that affect the positive valence of the task. Cost, in contrast, is best thought of as those factors (such as anticipated anxiety and anticipated cost of failure) that affect the negative valence of the activity. (Eccles \& Wigfield, 1995, S. 216)

Der Netto-Wert einer Aufgabe, der darüber entscheidet, ob das Individuum diese auch wirklich bearbeiten will bzw. welche Aufgabe es aus einer Auswahl letztlich wählt, hängt demnach auch von einer Kosten-Nutzen-Analyse ab: Je höher die relativen Kosten sind, desto eher entscheidet sich das Individuum gegen ein Engagement (vgl. Barron \& Hulleman, 2015, S. 505; Wigfield \& Eccles, 1992, S. 291-292). Eccles (2005, S. 113) postuliert drei Arten von Kosten: a) die erforderliche Anstrengung, die für eine erfolgreiche Bearbeitung nötig ist (effort cost), b) die Gelegenheitskosten (opportunity cost), also die fehlende Möglichkeit, durch die Bearbeitung einer vorliegenden Aufgabe andere, ebenfalls attraktive Aufgaben zu bearbeiten sowie c) die negativen affektiven Zustände (z. B. Angst, Ärger, Frustration, Stress), die sich allenfalls mit Schwierigkeiten bei der Bearbeitung oder im Falle eines Misserfolges einstellen könnten (psychological cost).

$\mathrm{Zu}$ letzteren zählt Eccles (2005, S. 112) Dynamiken und Prozesse, die Covington (1992) im Kontext des Selbstwert-Konstrukts (self-worth, self-esteem) beschrieben hat: Kinder, Jugendliche und Erwachsene sind angesichts der Bedeutung, die fähigkeitsbezogene Evaluationen, soziale Vergleiche und Wettbewerbe in Unterricht, Schule und Arbeitsstellen genießen, ständig darauf bedacht, ein positives Selbstbild eigener Kompetenz zu etablieren bzw. aufrecht zu erhalten und entwickeln aus diesem Bedürfnis heraus sowohl negative Affekte und Emotionen als auch Strategien zum Schutz des eigenen «sense of competency, respect, and self-acceptance» (Covington, 1992, S. 74) (vgl. auch Abschnitt 5.3.3.1). So antizipieren sie etwa allfällige Frustration oder den Ärger über sich selbst bei 
einem möglichen schleppenden Vorankommen, bangen davor, bei einem Misserfolg von den Peers ausgelacht, von der Lehrkraft vor der Klasse beschämt oder von den Eltern ausgeschimpft zu werden, haben aber bei entsprechenden Gruppennormen vielleicht auch davor Angst, bei einem eventuellen Erfolg von den Peers als «Streber*in» bezeichnet und abgelehnt zu werden oder befürchten Diskriminierungen und Zurückweisungen beispielsweise bei der Wahl vermeintlich nicht-geschlechtsadäquater Aktivitäten (vgl. Eccles, 2005, S. 112; Heckhausen \& Heckhausen, 2010, S. 454). Als Strategien zum Selbstwertschutz bieten sich gemäß Covington (1992, S. 74-103) und der Self-Enhancement-Theorie (zusf. Sedikides \& Gregg, 2008) u. a. Ausflüchte, Ausreden, Abwertungen des bedrohlichen Faches oder der Lehrkraft, Handlungsaufschübe oder einfach das Meiden entsprechender Aktivitäten an (vgl. auch Wigfield \& Cambria, 2010, S. 1920). Die bei weitem am stärksten beforschte Facette von psychological cost ist Leistungsangst (general anxiety bzw. test anxiety), die, wie Wigfield, Eccles, et al. (2015, S. 8) die Befundlage zusammenfassen, bei Betroffenen wegen der zunehmenden Dichte an evaluativen Situationen im Unterricht und den damit einhergehenden sozialen Vergleichen und potentiellen Misserfolgserlebnissen über die Schuljahre zunimmt und, wie Zeidner (2014) zeigt (vgl. auch Pekrun \& Götz, 2006; Rost, D. H., Schemer \& Sparfeldt, 2018), zu mitunter starken Beeinträchtigungen in Lern- und Leistungssituationen führt:

[...] anxious students tend to be easily distracted on cognitive tasks, experience difficulty in comprehending relatively simple instructions and questions, and also have difficulty organizing or recalling relevant information during the task. [...] highanxious students express concern about the consequences of not performing cognitively at a satisfactory level and embarrassment at probable failure. (Zeidner, 2014, S. 583)

Trotz der erklärtermaßen großen Bedeutung der Kosten-Komponente insbesondere für Entscheidungen, die stets sowohl von positiven wie negativen Merkmalen der ins Auge gefassten Aktivität beeinflusst werden (vgl. Abschnitt 3.1), hat diese in der empirischen Forschung zum Erwartungs-Wert-Modell von Eccles und Kolleg*innen weit geringere Aufmerksamkeit erfahren als die anderen WertKomponenten (vgl. de Brabander \& Martens, 2014, S. 30; Flake, Barron, Hulleman, McCoach \& Welsh, 2015; Wigfield \& Cambria, 2010, S. 5). Barron und Hulleman (2015) schreiben dies dem unklaren konzeptionellen Status zu, den perceived cost gegenüber den anderen Wert-Komponenten, aber auch bezüglich der Erwartungs-Komponente einnehme: 
[...] the work of Eccles and her colleagues remains largely silent on how to effectively measure cost or how researchers should weight the positive and negative subcomponents of value into an overall measure. Instead, their work concentrates on evaluating the positive subcomponents. (Barron \& Hulleman, 2015, S. 505)

Sie selber plädieren auf der Basis von neueren qualitativen und quantitativen Forschungsbefunden (zusf. Barron \& Hulleman, 2015, S. 506-508), die cost als konzeptionell ähnlich zu wahrgenommener Aufgabenschwierigkeit begreifen, dafür, Kosten nicht wie Eccles und Kolleg*innen als Facette von value, sondern als dritte Hauptkomponente des Motivationsmodells zu betrachten - im Sinne eines «Expectancy-Value-Cost-Modells» - wobei Kosten in der Bedeutung von «zu schwierig», «zu umfangreich», «zu zeitraubend» sowie «zu bedrohlich» von Anfang an als Barriere bzw. als hinderlicher Aspekt einer Aufgabe konzipiert werden, die ein Zuviel an antizipiertem Aufwand, Verlust an Zeit oder vermuteten negativen Affekten ausdrücken:

[...] we recommend writing cost items that capture a negative appraisal from the outset (e.g., 'This class is too challenging.'). To agree that a class is too challenging suggests it has surpassed a critical threshold and that it is now overwhelming and is perceived to have cost. Measuring cost objectively as the amount of effort or task difficulty alone is not enough. To be perceived as cost, it must be perceived negatively by the respondent. (Barron \& Hulleman, 2015, S. 507)

Neben den Fragen «Can I do this task?» und «Do I want to do this task and why?» (Wigfield, Eccles, et al., 2015, S. 3) stellt sich dem Individuum bei der Aufgabenwahl in dieser Perspektive eine dritte: «Am I free of barriers that prevent me from investing my time, energy, and resources into the activity?» (Barron \& Hulleman, 2015, S. 508). Dieses, so argumentieren die Autoren, könne die beiden ersten Fragen zwar überzeugt mit «ja» beantworten, aber noch immer unmotiviert sein, die Aufgabe in Angriff zu nehmen, wenn die dritte von ihm mit «nein» beantwortet werde: «If we only ask two of these three questions, the scope of our models to predict and understand motivation will be limited» (Barron \& Hulleman, 2015, S. 508). Erste vorläufige Befunde eigener Forschungsanstrengungen unter dieser Perspektive zeigten, so die Autoren, dass a) sich die expectancy-, value- und costKonstrukte faktorenanalytisch als klar distinkt erwiesen, b) expectancy und task value untereinander wie gewöhnlich positiv korrelierten, beide aber wie vorausgesagt negativ mit cost assoziiert seien, und c) sich die drei Konstrukte bei einer simultanen Berücksichtigung in Regressionen oder Pfad-Modellen so verhielten, dass expectancy der stärkste positive Prädiktor von Testleistungen, aber unverbunden mit fortgesetztem Interesse am Thema sei, dass task value sich dahingegen 
genau umgekehrt als stärkster Prädiktor für fortgesetztes individuelles Interesse am Thema, aber unverbunden mit den Testleistungen herausstelle und sich cost erwartungskonform sowohl für Testleistungen als auch fortgesetztes individuelles Interesse als negativer Prädiktor erweise (vgl. Barron \& Hulleman, 2015, S. 507-508).

Wie beispielsweise auch Trautwein et al. (2012) an einer Stichprobe von über 2'500 Gymnasiastinnen und Gymnasiasten in Deutschland zeigen, entpuppt sich die task value-Komponente mit Blick auf Leistungsmaße (Testleistungen, Zeugnisnoten) tatsächlich meist nicht mehr als prädiktiv, sobald die expectancyKomponente ebenfalls in die Prädiktionsmodelle einbezogen wird. In ihrer Studie ergab sich allerdings auch, dass die expectancy-Komponente zu einem noch stärkeren Prädiktor für die Testleistungen in Mathematik und Englisch wurde, sobald ein expectancy $x$ value product term (im Sinne Atkinsons) in die Regressionsgleichungen integriert wurde ${ }^{22}$. Anders als die expectancy-Komponente fungiert die value-Komponente dahingegen konsistent als starker Prädiktor für das langfristige individuelle Interesse an Fächern und Fachinhalten (z. B. Harackiewicz et al., 2008), die Absicht, in der Zukunft ein Fach zu studieren (z. B. Crombie et al., 2005; Eccles et al., 1993) sowie für die tatsächliche Entscheidung, bestimmte sportliche und musische Freizeitaktivitäten und Lehrgänge (namentlich Schwerpunktfächer in der Sekundarstufe I und II sowie Studienfächer an einer Hochschule) zu wählen (z. B. Meece, Wigfield \& Eccles, 1990; Simpkins, Fredricks \& Eccles, 2012; Simpkins et al., 2015b) - sogar oft Jahre später (vgl. Durik et al., 2006; Musu-Gillette, Wigfield, Harring \& Eccles, 2015) - oder für die Entscheidung von Erwachsenen, bestimmte berufliche Karrieren zu ergreifen (z. B. Farmer, Wardrop \& Rotella, 1999). Ebenso ist der zugeschriebene Wert bei simultanem Einbezug beider Komponenten in der Regel der stärkere Prädiktor für das Lernengagement und die Persistenz (vgl. Nagengast et al., 2011; Trautwein \& Lüdtke, 2007). Ferner erweisen sich subjektive task values ebenso wie expectancies als ausgeprägt fachspezifische Konstrukte, insofern als sie zwar untereinander domänenintern positiv assoziiert sind, aber bestenfalls moderat mit Kontroll- und Wert-Einschätzungen bzw. -Überzeugungen in anderen Domänen korrelieren (vgl. Denissen, Zarrett \& Eccles, 2007). Wie oben bereits im Kontext des Internal/External-Frame-of-Reference-Modells von Marsh (1986b) erläutert

\footnotetext{
${ }^{22}$ Auch wenn dieser Erwartungs-mal-Wert-Interaktions-Term lediglich einen kleinen Teil der Varianz aufzuklären vermochte und der Effekt womöglich nur wegen der großen Stichprobe sichtbar wurde (ähnlich bei Nagengast et al., 2011), so macht dieser mit Blick auf die Überlegungen Atkinsons (vgl. Abschnitt 5.1) theoretisch durchaus Sinn: «if one does not value a task, then expecting to do well on it may not be a sufficient reason to engage in it» (Wigfield et al., 2016, S. 59).
} 
(vgl. Abschnitt 5.3.2.2), sind vor allem zwischen Mathematik und Sprache die Korrelationen zwischen den Leistungstestergebnissen und Noten bedeutend höher als zwischen den korrespondierenden domänenbezogenen Kontroll- und WertÜberzeugungen (vgl. Trautwein et al., 2012). Während bei kleinen Kindern die domänenspezifischen Kontroll- und Wert-Überzeugungen noch weitgehend unabhängig voneinander zu sein scheinen - «children might pursue some achievement activities in which they are interested regardless of how good or bad they think they are at the activity» (Wigfield, Eccles, et al., 2015, S. 9) -, werden die Assoziationen mit zunehmendem Alter bzw. höherer Klassenstufe laufend enger (z. B. Wigfield et al., 1997). Es ist denn auch nicht weiter erstaunlich, dass ebenso wie die Kontrollüberzeugungen auch die Wertüberzeugungen mit zunehmendem Alter bzw. Klassenstufe abnehmen (vgl. Jacobs et al., 2002; Wigfield et al., 1997). Dabei deuten die meisten Befunde darauf hin, dass die Kausalrichtung primär von den Kontroll-Überzeugungen zu den Wertüberzeugungen verläuft (zsf. Wigfield, Eccles, et al., 2015, S. 9): Kinder messen denjenigen Fächern und Aktivitäten einen höheren Wert zu, bei denen sie sich als erfolgreich und fähig erleben (u. a. Denissen et al., 2007; Marsh, Trautwein, Lüdtke, Köller \& Baumert, 2005). So zeigten beispielsweise Jacobs et al. (2002) in einer Studie mit 761 Schülerinnen und Schülern, die vom Schuleintritt bis zur Vollendung der Sekundarstufe I begleitet wurden, dass Veränderungen in den Kompetenzüberzeugungen in Mathematik, sprachlichen Fächern sowie Sport über $40 \%$ der Varianz in den Veränderungen des Wertes erklärte, den die Kinder und Jugendlichen diesen Fächern beimaßen. In Einklang mit den Postulaten der Selbstbestimmungstheorie (vgl. Ryan \& Deci, 2002, 2016) scheinen sodann die Beziehungen der expectancy-Komponente zu den Wert-Facetten intrinsic value und attainment value stärker zu sein, als zu derjenigen des utility value (Eccles \& Wigfield, 1995): «Thus the more intrinsic aspects of value (interest and importance) relate more closely to children's competence related beliefs» (Wigfield \& Eccles, 2002).

\subsection{Unterschiedliche Task Values: Implikationen für die Analyse elterlicher Bedeutsamkeitszuschreibungen gegenüber dem Kind}

Nebst elterlichen Kontrollregulationen in Form von evaluativen Feedbacks (vgl. Abschnitte 5.3.3.2 und 5.4) interessiert in der vorliegenden Studie auch, wie die Eltern in der Zeit vor dem Übertrittsentscheid versucht haben, ihre Kinder für ein bestimmtes Lern- und Leistungshandeln durch verbale Bedeutsamkeitszuschreibungen zu motivieren. Entweder ausgelöst durch Bitten des Kindes nach 
Ratschlägen bei Schwierigkeiten mit Lernaufgaben und anderen Unterrichtsangeboten sowie mit Peers und Lehrkräften (z. B. «Papa, wie soll ich das am besten machen?») oder aber durch Probleme, die der Elternteil selbst im schulbezogenen Handeln des Kindes wahrnimmt (z. B. «Unsere Tochter erkennt noch immer nicht, dass es für unser Familienleben wichtig ist, dass sie nicht am Sonntagabend noch ihre Hausaufgaben für Montag erledigt»), übermitteln diese ihrem Kind als «interpreters of reality» (Jacobs \& Bleeker, 2004, S. 7-8; Jacobs \& Eccles, 2000, S. 426) (vgl. Abschnitt 2.2.2.4) in solcherlei Wertregulationen, Botschaften darüber, welche Relevanz sie mit Blick auf dessen Kompetenzentwicklung sowie auf anstehende Leistungsereignisse in bestimmten schulbezogenen Aktivitäten erkennen (vgl. Abschnitt 2.2, insb. Abbildung 2.1), und inwiefern sie möchten, dass das Kind sich entsprechend verhält. Dabei sehen sich die Eltern in solchen Kommunikationssituationen nicht nur mit der Schwierigkeit konfrontiert, a) die Aufmerksamkeit ihres Kindes auf diese spezifischen Aspekte zu lenken, sondern b) auch die von ihnen in diesen erkannte Bedeutsamkeit - den task value - deutlich $\mathrm{zu}$ machen und c) dies kommunikativ so zu gestalten, dass das Kind gewillt ist, entsprechend zu handeln (vgl. Abschnitt 5.7). Wie bei den Kontrollregulationen besteht das zu lösende Problem für die Eltern im Kern darin, ein aus der Perspektive ihres Kindes fremdzugeschriebenes Lern- oder Leistungsziel ( $\mathrm{X}$ ist für dich bedeutsam» [X ist somit ein task]) mittels einleuchtenden Argumenten («..., weil du...» [task value]) und einem internalisierungsförderlichen Kommunikationsmodus so überzeugend darzulegen, dass es dem Appell («Merk dir das!») nicht nur Gehör schenkt, sondern sich das Ziel und dessen relative Bedeutung zu eigen macht - dass aus dem elterlichen task value ein subjective task value des Kindes wird.

Auf der Basis dieser Überlegungen lässt sich analog zum Vorgehen bei den Kontrollregulationen (vgl. Abschnitt 5.4) der folgende Frame konstruieren, mit dessen Hilfe sich die in den Interviews sprachlich unterschiedlich realisierten Handlungsepisoden im Hinblick auf die Analyse bezüglich der genannten drei Aspekte vereinheitlichen lassen:

«Merke dir: Es ist bedeutsam, dass du ... [schulischer task, den das Kind verwirklichen soll], weil du ... [vom Elternteil zum Ausdruck gebrachter task value]».

Die Leerstellen des Frames werden im Rahmen des Codierprozesses (vgl. Abschnitt 6.4.2) in möglichst großer Nähe zu den Originaläußerung der Elternteile über Interpretationsleistungen ergänzt. Das Ziel besteht darin, den propositionalen Gehalt der elterlichen Wertregulationen möglichst adäquat - so, wie ihn die Elternteile mit hoher Wahrscheinlichkeit dem Kind übermitteln wollten - zu rekonstruieren (vgl. Linke et al., 2004, S. 210-216). Am Beispiel der folgenden 
bereits in Abschnitt 1.2 kurz erläuterten wertbezogenen Handlungsepisode der Mutter S12 aus dem Interview G1 lässt sich illustrieren, wie das Framing einer wertbezogenen Interviewsequenz vonstattengeht:

I: Diskutieren Sie denn den Übertritt mit Ihrem Sohn?

S12: Ja, haben wir schon ziemlich oft darüber diskutiert (lacht).

I: Und wie spielt sich das dann ab?

S12: Ja, dass wir eben- also das letzte Mal nach dieser Rechnungsprüfung war ich ziemlich auf 180 (lacht) und habe gesagt: «also so geht das nicht weiter, also jetzt müssen wir irgendetwas machen». Das war ein Abend - ich habe dann auch nicht mehr geschlafen und so; es hat mich total beschäftigt, vor allem, weil ich- ja, man ist ja auch so ausgeliefert, nicht wahr, man kann ja dann nicht irgendwie reagieren. Und- nein, wir diskutieren mit ihm, dass das wichtig ist für die Zukunft, und dass man halt arbeiten muss, obwohl - er ist ja selbständig, er macht es, aber eben, vielleicht - ich weiß es nicht - vielleicht zu wenig. Das kann ich zu wenig einschätzen. (Interview G1, 00:53:32)

Zur Füllung der ersten Leerstelle wird ermittelt, welches Lern- oder Leistungsziel (bzw. welchen task) der Elternteil gegenüber dem Kind als bedeutsam herausstreicht und zur Verwirklichung nahelegt. In der vorliegenden Sequenz findet sich dieses in der indirekten Rede, die von «wir diskutieren mit ihm» eingeleitet wird: «dass man halt arbeiten muss». Für die Übersetzung in den Frame stellt sich zunächst die Frage, was damit gemeint ist. Da die Mutter im Ko-Text von der Selbständigkeit des Arbeitens spricht und die Episode offenbar im Umfeld unbefriedigender Prüfungsnoten zustande kam, kann angenommen werden, dass sie mit «arbeiten» die Hausaufgabenerledigung und Prüfungsvorbereitungen zuhause meint. Sodann stellt sich die Frage, was sich am Arbeiten gemäß der Mutter verändern soll («so geht das nicht weiter»). Die Anmerkung «er macht es, aber eben, vielleicht - ich weiß es nicht - vielleicht zu wenig» lässt vermuten, dass sie gegenüber ihrem Sohn in erster Linie ein erhöhtes zeitliches Investment hervorstrich. Der erste Teil der geframten Aussage lautet demnach: «Es ist bedeutsam, dass du ein größeres Engagement bei Hausaufgaben und Prüfungsvorbereitungen zeigst».

Der zweite Teil des Frames, die Begründung bzw. der von den Eltern dem Kind dargelegte task value, der letztlich zum Ausdruck bringt, wie hoch das Ziel nach ihrer Meinung in der Präferenzordnung des Kindes rangieren sollte, findet sich im ersten Teil der indirekten Rede: «wir diskutieren mit ihm, dass das wichtig ist für die Zukunft». Auch hier stellt sich die Frage, worauf sie dabei genau anspielt: 
meint sie die weitere berufliche Zukunft oder meint sie die zu diesem Zeitpunkt acht Monate vor dem Entscheid - noch unklare Zuteilung in die Sekundarstufe I? Der Ko-Text, insbesondere die diesen Abschnitt auslösende Frage der interviewenden Person, deutet stark auf Letzteres hin: es ist «wichtig» für den in naher Zukunft erfolgenden Übertrittsentscheid.

Nun stellt sich weiter die Frage, welche Bedeutsamkeit die Mutter ihrem Sohn hierbei zur Internalisierung anbietet. Was meint sie damit, wenn sie von «wichtig» spricht? Steht mit Blick auf die task value-Klassifikation der Arbeitsgruppe um Eccles (2005) die Nützlichkeit bzw. Zweckmäßigkeit (vgl. Abbildung 5.4) im Vordergrund («weil du so den Übertritt schaffen kannst»)? Oder überwiegt die Intention, dem Sohn im Sinne der Konzeption des attainment value dem vorliegenden task («größeres Engagements bei Hausaufgaben und Prüfungsvorbereitungen») eine Relevanz nahezulegen, die über eine bloße MittelZweck-Relation hinausgeht und für etwas steht, was der Sohn aus ihrer Sicht als maßgeblich für sein Selbstbild - als identitätsrelevant - anerkennen sollte: «weil du das als zukünftiger Sekundarschüler einfach musst»? Das Team der Codierenden dieser Studie hätte aufgrund der Festlegungen im Kategoriensystem (vgl. Abbildung 5.4) im vorliegenden Beispiel wohl klar für Letzteres votiert: Das Verb müssen sowie das Adjektiv wichtig sind starke Indikatoren dafür, dass die Mutter hierbei von einem essenziellen Prinzip spricht, welches ihr Sohn beherzigen möge. Sodann hätten die Codierenden im Rahmen der inhaltlich strukturierenden qualitativen Inhaltsanalyse (vgl. Abschnitt 6.4.2) noch weiter danach differenziert, ob die Mutter ihrem Kind in der vorliegenden Bedeutsamkeitszuschreibung im Sinne persönlicher Identität (vgl. Abschnitt 5.5.1.2) eher einen internalen Lokus («weil du einfach so bist bzw. dich so sehen solltest») oder ob sie ihm im Sinne sozialer Identität, der Zugehörigkeit zu sozialen Gruppen (vgl. Jacobs \& Eccles, 2000, S. 411-413), eher einen externalen Lokus nahelegt und somit auf Normen bzw. Rollenerwartungen (vgl. Abschnitt 1.2) verweist, denen es entsprechen soll («du als Junge», «du als unser Sohn», «du als angehender Gymnasiast», «du als Sportler» etc.). Im vorliegenden Beispiel handelt es sich um Letzteres: Die Mutter S12 dürfte ihrem Sohn signalisieren, dass es sich für ihn (in seiner Rolle) als angehender Sekundarschüler so gehöre.

Wie das weiter unten abgebildete Kategoriensystem (vgl. Abbildung 5.4) illustriert, ließen sich bei den Begründungen, die auf die Zweckmäßigkeit eines Ziels verwiesen, ebenfalls ein internaler und ein externaler Lokus unterscheiden. Begründet der Elternteil mit internaler Zweckmäßigkeit, signalisiert er dem Kind, dass es die betreffende Aktivität als instrumentell zur Befriedigung seiner persönlichen kognitiven, motivational-affektiven und/oder behavioralen Ziele und Bedürfnisse erachten solle (z. B. «weil du es so besser verstehst», «weil du dich 
dann freuen kannst», «weil es dann leichter vonstatten geht»). Demgegenüber steht bei externaler Zweckmäßigkeit die Dienlichkeit eines Handelns zur Erlangung von sozial gesetzten Zielen bzw. zur Befriedigung sozialer Bedürfnisse und Motive im Vordergrund: z. B. «weil du dann bessere Noten hast», «weil du so den Übertritt schaffst», «weil du dann bewundert wirst».

Es stellt sich nun die Frage, inwiefern intrinsic value und perceived cost, die beiden weiteren von Eccles und Kolleg*innen postulierten Facetten des subjective task value, in verbalen Wertregulationen eine Rolle spielen und wie diese gegebenenfalls realisiert werden.

Es ist denkbar, dass Eltern auch den intrinsic value, den sie einer schulbezogenen Aktivität zuschreiben, verbal explizit gegenüber dem Kind zum Ausdruck bringen, indem sie den Spaß und/oder das situative Interesse, das sie selber empfinden oder aber beim Kind wahrzunehmen glauben, verbalisieren: «Es ist bedeutsam, dass du ... [task, den das Kind verwirklichen soll], weil du dabei Spaß empfindest/empfinden wirst» oder «..., weil du es spannend finden wirst». Allerdings wird bei dieser Facette, die von Eccles als genuin affektives Konstrukt konzipiert ist, welches in hohem Maß von «core aspects of the self, such as temperament, personality, motivational orientations» (Eccles, 2005, S. 111) beeinflusst ist und zunächst ohne größere rationale Erwägungen einfach empfunden wird, noch stärker als bei den anderen Wert-Facetten deutlich, dass Eltern dem Kind einen spezifischen task value, den sie selber erkennen, verbal zwar zur Internalisierung anbieten können, ihr Kind aber unter Umständen eine gänzlich andere Bedeutsamkeit im task wahrnimmt. Dies dürfte insbesondere dann zutreffen, wenn die Eltern Spaß nicht nur im Sinne eines assertiven Sprechakts (vgl. Abschnitt 2.2.2.4) laut denkend konstatieren («ich habe Spaß» bzw. «wir haben Spaß»), sondern als direktiven Sprechakt (vgl. Searle, 1976) bzw. als Appell realisieren («Merke dir!»). Spaß empfindet man spontan, ebenso wie situatives Interesse. Andere können einem allenfalls durch das augenscheinliche Vergnügen, das ihnen die Beschäftigung mit der Aufgabe offenbar bereitet, als animierende Modelle (vgl. Bandura, 1992) dienen («meiner Mutter bereitet das offenbar Spaß, also muss was dran sein»; vgl. parents role modelling behavior, Abbildung 4.1, Box F) oder aber durch ihre Einladung, ebenfalls mitzumachen, Spaß hervorrufen (encouragement of various activities, vgl. Abbildung 4.1, Box G). Mit verbalen Appellen aber, man soll es tun, weil es Spaß mache oder interessant sei, transformiert sich der von den Eltern konstatierte und zur Übermittlung gemeinte intrinsic value («Es ist für mich bedeutsam, weil es mir einfach Spaß macht. Das möchte ich auch meinem Kind nahebringen») in einen utility value bzw. eine Wertregulation, die mit internaler Zweckmäßigkeit (vgl. Abbildung 5.4) operiert, also dem 
Kind im Sinne eines Ratschlags als Mittel zum Zweck eines positiven affektiven Zustands präsentiert wird: «Es ist bedeutsam, dass du ... [task, den das Kind verwirklichen soll], weil du dabei Spaß empfinden wirst».

Bevor dem Problem noch eingehender Beachtung geschenkt wird, wonach sich task values im Zuge des Appellierens von den Kindern nicht so rezipiert werden, wie sie von den sendenden Eltern intendiert sind (vgl. Abschnitt 5.6.2), soll nun die Komponente perceived cost und deren Fremdregulation durch Eltern sowie das Kategoriensystem für die inhaltliche Analyse von fremdzugeschriebenen WertBegründungen (vgl. Abbildung 5.4) als Ganzes ins Blickfeld gerückt werden.

Als perceived cost werden von Eccles und Kolleg*innen negativ-valente Bedeutsamkeitseinschätzungen bezeichnet: Aspekte einer Aktivität, die einen diese grundsätzlich eher meiden lassen (vgl. Abschnitt 5.5.1.4). Eltern motivieren ihre Kinder nicht nur zu bestimmten Handlungsweisen. Auf der Grundlage ihrer Lebenserfahrung sowie ihrer ethisch-moralischen Vorstellungen versuchen sie auch, diese auf Fehler, Gefahren, Umwege, Nachteile, begrenzte Ressourcen - kurz auf Restriktionen (vgl. Esser, 1999a, S. 238) - aufmerksam zu machen (vgl. Abschnitte 2.1 und 2.2.2.4). Eltern dürften ihre Kinder im Rahmen ihres schulbezogenen Unterstützungshandelns vor solchen Restriktionen warnen («weil du sonst...), selber solche deklarieren («weil du das nicht darfst») sowie allenfalls auch mit Konsequenzen drohen, wenn Restriktionen übertreten werden («weil du sonst ... gewärtigen musst»).

An der bereits mehrmals als Beispiel herangezogenen Handlungsepisode aus dem Interview G1 mit der Mutter S11 lässt sich dies illustrieren:

S11: Ich habe das Gefühl- er möchte es irgendwie noch nicht ganz wahrhaben. So kommt es mir vor. Er möchte vielleicht schon- er denkt: «Wenn ich mich jetzt ein bisschen engagiere, komme ich schon in die Sek A, aber es muss noch nicht, hat noch Zeit.» Ich weiß nicht, wo er steht mit seinem Kopf. Das frage ich mich manchmal auch. Aber... es nervt mich manchmal auch ein bisschen, wenn ich sage: «K03, wach auf!» (lacht) [...] Er muss ja kein Weltmeister werden. Er muss sich ja weiß Gott nicht übermäßig engagieren - er muss mir keine Sechsen [in der Schweiz die höchste Note] nach Hause bringen. Aber ich sage: «K03, du bist fähig, Fünfen nach Hause zu bringen. Warum kommst du manchmal mit einer Viereinhalb oder Vier nach Hause? Nur weil du es ein bisschen locker genommen hast?» Er: «Ja, ja, das nächste Mal dann». Das gibt es nicht. Hätte er mindestens Probleme, würde ich das akzeptieren, immerhin eine Vier, nicht wahr. Wenn er jetzt so einer wäre. Deswegen nervt es mich, wenn auch die Lehrerin und der Lehrer sagten: «Du kannst es, aber du willst es einfach nicht wahrhaben». (Interview G1, 00:40:52) 
Nebst der in Abschnitt 5.4 erläuterten Kontrollregulation findet sich in dieser Sequenz auch folgende - bereits in den Frame übertragene - wertbezogene Regulation der Mutter:

Merke dir: Es ist bedeutsam, dass du dir der Notwendigkeit konstant guter Noten und eines entsprechenden Lernengagements bewusst wirst, weil du sonst den Übertritt in die Abteilung A nicht schaffen wirst.

Der task bzw. das schulbezogene Ziel, welches die Mutter ins Zentrum ihres Appells stellt, besteht in einer veränderten Einstellung, die ihr Sohn gegenüber dem Lernen und Leisten speziell in der Phase vor dem Übertritt einnehmen soll. In der im Weil-Satz ausgedrückten Begründung wird deutlich, dass sie ihn vor negativen Konsequenzen warnt, ihrem Sohn gegenüber also mit möglichen Kosten argumentiert, die sie wahrnimmt.

Wie lassen sich die von der Mutter S11 ausgedrückten Kosten charakterisieren? Eccles (2005, S. 113) postuliert drei Arten von Kostenüberlegungen, welche ein Individuum mit Blick auf eine anstehende Aufgabe anstellen kann (vgl. Abschnitt 5.5.1.4): a) erforderliche Anstrengungen (effort cost), b) Kosten, die sich aus Entgangenem ergeben (opportunity cost) sowie c) negative affektive Zustände (z. B. Angst, Ärger, Frustration, Stress) (psychological cost). Worum handelt es sich beim vorliegenden Beispiel?

Je mehr Beispiele von Episoden im Zuge der Entwicklung eines Kategoriensystems zur Analyse elterlicher Wertregulationen herangezogen wurden, desto klarer wurde, dass sich eine Klassifizierung der von den Eltern als Begründungen vorgebrachten Restriktionen mit den drei Facetten der Kosten-Komponente von Eccles und Kolleg*innen kaum trennscharf und reliabel bewerkstelligen lässt. Anstelle der Schaffung zusätzlicher Kostenfacetten entschied sich das Entwicklungsteam vor dem Hintergrund des expectancy-value-cost-Ansatzes von Barron und Hulleman (2015), die Kostenkomponente als gleichrangig zur Erwartungsund Wert-Komponente zu verstehen (vgl. Abschnitt 5.5.1.4) und elterliche Begründungen, die Aussagen über Restriktionen beinhalteten, als negative Ausprägungen der positiven Wert-Komponenten attainment value und utility value zu konzipieren. Am Weil-Satz des obigen Beispiels, «weil du sonst den Übertritt in die Abteilung A nicht schaffen wirst», lässt sich dieses Prinzip verdeutlichen: Durch die beiden Wörter «sonst» und «nicht» wird in der geframten Begründung nicht nur die Restriktion, sondern auch die Instrumentalität bzw. der utility value deutlich: «Es ist bedeutsam, dass du X tust, um Y nicht zu erleben». Analog dazu hat sich gezeigt, dass die Elternteile nicht nur im Sinne des Eccles'schen Konstrukts des attainment value dem Kind positiv-valente identitätsbezogene Aspekte zur Begründung der Bedeutung einer Aktivität vor Augen führen («Es ist bedeutsam, dass du X tust, weil du das mit Blick auf soziale Normen und 
Erwartungen einfach musst» bzw. «Es ist bedeutsam, dass du X tust, weil du das mit Blick auf deine Persönlichkeitsmerkmale kannst/tun solltest»), sondern auch negativ-valente, die signalisieren, dass ein anderes Handeln bzw. Leistungsziel aus externalen oder internalen Gründen ausgeschlossen sei («Es ist bedeutsam, dass du X tust, weil du Y mit Blick auf soziale Normen und Erwartungen einfach nicht darfst» bzw. «Es ist bedeutsam, dass du X tust, weil du Y mit Blick auf deine Persönlichkeitsmerkmale nicht kannst/tun solltest») (vgl. Abbildung 5.4) ${ }^{23}$.

\subsubsection{Die Vermittlung von Task Values: Ein Kategoriensystem}

Abbildung 5.4 illustriert das Ergebnis der theorie- und datengeleiteten Entwicklung eines Kategoriensystems zur Codierung der verbalen elterlichen Wertregulationen entlang sogenannt wertbezogener Begründungstypen (vgl. auch Abschnitt 6.4.2.1). Wie sich zeigt, versuchten die Elternteile ihr Kind im Kontext eines unsicheren Übertritts von der Bedeutung bestimmter schulbezogener Aktivitäten zu überzeugen, indem sie ihnen im Sinne des Konstrukts des utility value entweder die Zweckmäßigkeit (Code «O» für Opportunität) oder im Sinne des Konstrukts des attainment value die Wesentlichkeit (Code «E» für Essentialität) einer bestimmten schulischen Aktivität bzw. eines bestimmten Lern- oder Leistungsziels argumentativ vor Augen führen. Wie Abbildung 5.4 weiter zeigt, besteht die Dimension «Aufgabenwert, den der Elternteil vom Kind erkannt haben möchte» nur aus diesen beiden Facetten. Wie oben vermutet, fanden sich im Datenmaterial keine Handlungsepisoden, die inhaltlich die Schaffung einer eigenständigen Facette zum intrinsic value-Konstrukt gerechtfertigt hätten. Sobald ein Elternteil die Bedeutsamkeit einer schulischen Aktivität gegenüber dem Kind mit Spaß begründet, wandelt sich der Charakter der Begründung durch die intendierte Fremdregulation und die damit einhergehende Verbalisierung bzw. Rationalisierung zu einem utility value («weil du dadurch Spaß haben wirst»), der mit Blick auf den Lokus, die zweite Dimension des Kategoriensystems, noch spezifizierter als internale Zweckmäßigkeit bezeichnet bzw. codiert werden kann.

\footnotetext{
${ }^{23}$ Gleichermaßen ließe sich im Prinzip (vgl. obige Ausführung zur Problematik von wertbezogenen Appellen) eine Begründung mit einem intrinsic value ( Es ist bedeutsam, dass du X tust, weil du dabei Spaß empfindest/empfinden wirst» bzw. «..., weil du es spannend finden wirst») invers ausdrücken: «Es ist bedeutsam, dass du X tust, weil du von Y abgestoßen bist/sein wirst»). Die Bedeutung einer bestimmten Aktivität würde dann nicht mit Freude, Lust und Interesse, die diese bereite, begründet, sondern durch ebenso tiefsitzende persönliche Aversionen, die mit alternativen Aktivitäten verbunden wären.
} 
Wie oben bereits erläutert, bezeichnet die Dimension Lokus (vgl. Abbildung 5.4) den Referenzbereich, auf den das Kind seine Aufmerksamkeit richten soll: bei einem internalen Lokus (Code «i») soll das Kind die Zweckmäßigkeit oder Wesentlichkeit mit Blick auf innere Zustände bez. seine Persönlichkeit erkennen, bei einem externalen Lokus (Code «e») auf äußere Anforderungen und Restriktionen.

Die dritte Dimension des Kategoriensystems, Valenz der Wertaussage (vgl. Abbildung 5.4) bezieht sich gemäß des oben bereits Erläuterten auf den approachvs. avoidance-Aspekt (vgl. Abschnitt 5.1): In positiv-valenten bzw. appetitiven Begründungen (Code «+») signalisiert der Elternteil, dass das Kind sich zwecks Annäherung an einen angenehmen oder erwünschten Zielzustand mit einer Aktivität beschäftigen soll. Bei negativ-valenten bzw. aversiven Begründungen (Code «-») verweist der Elternteil auf Kosten und Restriktionen.

Aus den drei Dimensionen gehen die im Kategoriensystem dargestellten acht möglichen Begründungstypen bzw. Argumentationsstrategien hervor, welche die Elternteile bei der Kommunikation der Bedeutsamkeit einer schulbezogenen Aktivität oder eines Leistungsziels gegenüber dem Kind einsetzen:

- Positive externale Zweckmäßigkeit (Code: Oe+): «X ist bedeutsam, weil du so einen erwünschten sozialen Status erreichst.»

- Negative externale Zweckmäßigkeit (Code: Oe-): «X ist bedeutsam, weil du sonst mit einem unerwünschten sozialen Status konfrontiert bist.»

- Positive internale Zweckmäßigkeit (Code: Oi+): «X ist bedeutsam, weil du so kognitiv, emotional und/oder behavioral profitierst.»

- Negative internale Zweckmäßigkeit (Code: $\mathrm{Oi}-$ ): «X ist bedeutsam, weil du sonst mit kognitiv, emotional und/oder behavioral negativen Folgen konfrontiert bist.»

- Positive externale Wesentlichkeit (Code: Ee+): «X ist bedeutsam, weil du das mit Blick auf soziale Normen einfach musst.»

- Negative externale Wesentlichkeit (Code: Ee-): «X ist bedeutsam, weil du etwas anderes mit Blick auf soziale Normen einfach nicht tun darfst.»

- Positive internale Wesentlichkeit (Code: Ei+): «X ist bedeutsam, weil du jemand bist, der das kann.»

- Negative internale Wesentlichkeit (Code: $\mathrm{Ei}-$ ): «X ist bedeutsam, weil du nicht jemand bist, der andere Optionen hat.»

Diese dreidimensionale Taxonomie stellt der einzige uns bekannte Versuch einer Klassifikation der Strategien dar, mit denen Eltern versuchen, ihr Kind verbal 


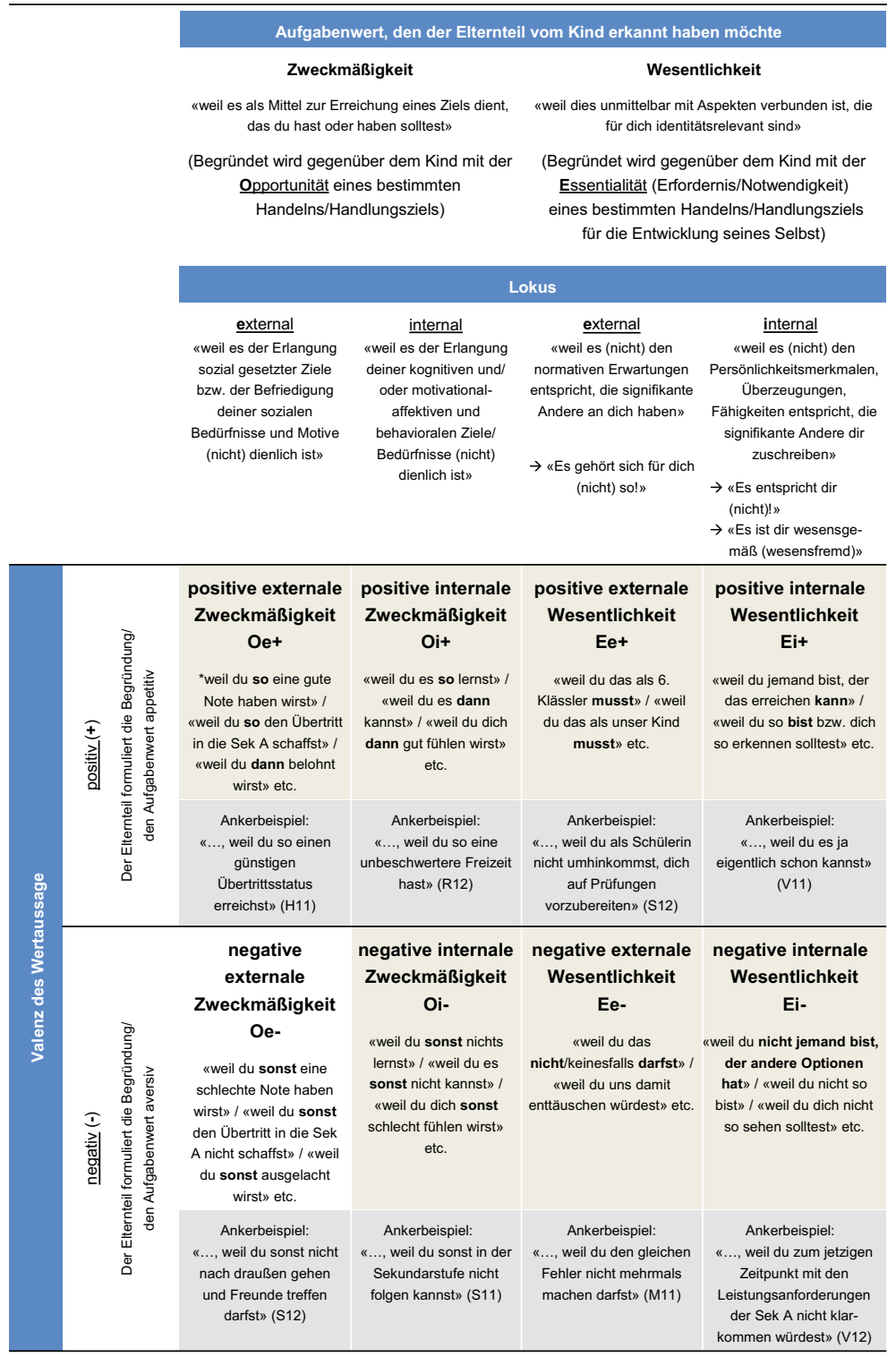

Abbildung 5.4: Kategoriensystem «Elterliche Strategien der verbalen Wertvermittlung» (sog. «wertbezogene Begründungstypen») 
vom Wert schulischer Aktivitäten und Ziele (von tasks) zu überzeugen. Auf unserer Suche nach bestehenden Beschreibungs- und Analysesystemen, die an der einflussreichen Konzeption der task values orientiert wären, wurden wir auch in der Forschung zur Unterrichtskommunikation bzw. zum Lehrpersonenfeedback diesbezüglich nicht fündig, obwohl Schülerinnen und Schüler im Rahmen des Klassenunterrichts oder der Lernberatung ${ }^{24}$ nicht selten verbal motiviert werden. Es kann angenommen werden, dass sich das vorliegende Schema auch zur Analyse verbaler Bedeutsamkeitszuschreibungen von Lehrkräften im Unterricht eignet (vgl. Abschnitt 8.4).

\subsubsection{Die Vermittlung von Task Values: Welche Strategien sind erfolgsversprechend?}

Wie bei den verbalen Kontrollregulationen (vgl. Abschnitt 5.4) drängt sich auch bei den elterlichen Wertregulationen die Frage auf, bei welchen dieser acht Argumentationsstrategien die Eltern sich berechtigte Hoffnungen machen können, dass sich ihr Kind bei deren Einsatz mit erhöhter Wahrscheinlichkeit von der Relevanz einer bestimmten Aktivität oder eines Ziels überzeugen lässt und bei welchen wohl weniger. Wie in diesem Kapitel zu zeigen sein wird, ist die Beantwortung dieser Frage angesichts einer beschränkten empirischen Befundlage schwierig und entsprechend tentativ. Die Zahl an Interventionsstudien zur Ermittlung der Effektivität relevanzsteigernder Strategien - sog. value interventions - nimmt zwar stetig zu, allerdings handelt es sich hierbei um ein junges Forschungsgebiet, dessen bisherigen Befunde mit der entsprechenden Vorsicht zu interpretieren sind (vgl. Hulleman \& Barron, 2016, S. 163-164). Obwohl drei Theoriefamilien

\footnotetext{
${ }^{24}$ Hattie und Timperley (2007) haben auf der Grundlage der Metaanalyse von Kluger und DeNisi (1996) zu den Effekten verschiedener Feedbackarten eine multidimensionale Konzeption wirksamen Lehrpersonenfeedbacks vorgelegt (Hattie \& Wollenschläger, 2014), die nicht nur darauf beruht, dass Schülerinnen und Schüler Informationen zum aktuellen Leistungstand bez. Aufgaben, Prozesse, Selbststeuerung und Persönlichkeitsmerkmale («How am I going?») - sog. Feed Back - erhalten, sondern in sog. Feed Up- und Feed ForwardMoves ebenso mit Informationen zu den (nächsten) Lernzielen in diesen Bereichen («Where am I going?») und zielführenden Strategien («Where to next?») versorgt werden (Hattie \& Timperley, 2007, S. 86). Die beiden letzteren Moves dürften Gewichtungen im Sinne der task values durch die Feedback-Gebenden und -Erhaltenden beinhalten. Die in der vorliegenden Arbeit untersuchten elternberichteten Handlungsepisoden können denn auch als Ganzes als Schilderungen von Rückmeldesituationen verstanden werden, in denen die Eltern von ihren Feed Back-Moves (elterliche Kontrollregulationen) sowie - oft noch im gleichen Satz - von ihre Feed Up- und Feed Forward-Moves (elterliche Wertregulationen) berichten.
} 
- die Expectancy-Value-Theorie, die Interesse-Theorie sowie die Selbstbestimmungstheorie - die Bedeutung des Wert-Konstrukts thematisieren und ein breiter Konsens in der didaktischen Fachliteratur und unter Lehrkräften herrscht, wonach beispielsweise der Lebensweltbezug schulischer Inhalte (relevance to students' lives) wichtig sei (z. B. Helmke, 2015, S. 223-224; Lipowsky, 2015, S. 71; Woolfolk \& Schönpflug, 2014, S. 408), ist mit entsprechender Forschung also noch immer kaum gesichert, welche spezifischen Merkmale didaktischer Maßnahmen und Settings die Wert-Wahrnehmung von Schülerinnen und Schülern tatsächlich positiv zu verändern vermögen. Solche «design-based studies» (Hulleman \& Barron, 2016, S. 161) sind insbesondere selten, wenn es um verbalsprachliche Wert-Regulationen - also «directly communicated information» (Canning \& Harackiewicz, 2015, S. 47) - geht und diese nicht im unterrichtlichen, sondern im häuslichen Kontext angesiedelt sind. Die im Folgenden herausgearbeiteten Empfehlungen für die Gestaltung von elterlichen Wertzuschreibungen haben denn auch bis zu einem gewissen Grad hypothetischen Charakter. Entlang der drei Ordnungskriterien, aus denen das Kategoriensystem aufgebaut ist, sollen auf der Grundlage der in den vergangenen Kapiteln dargestellten intraindividuellen Zusammenhänge zwischen task values und motivational-affektiven sowie behavioralen Prozessen Einschätzungen dazu vorgenommen werden, wie die wertbezogenen Botschaften der Eltern beschaffen sein sollten, damit Kinder diesen die nötige Aufmerksamkeit schenken, sie internalisieren und in entsprechendes Handeln umsetzen.

\subsubsection{Die Dimension Valenz der Wertaussage}

Die Dimension Valenz der Wertaussage verweist darauf, dass Eltern ihre verbalen Verhaltensförderungen im Sinne der Lerngesetze des operanten Konditionierens (vgl. Skinner, B. F., 1971, 1989) mit der sprachlichen Darbietung eines positiven oder eines negativen Verstärkers betreiben können. Im ersteren Fall (Code: «+») wird versucht, das Kind für eine veränderte Bedeutungszuschreibung hinsichtlich einer Aktivität oder eines Ziels zu gewinnen, indem ihm eine - aus der Perspektive der Eltern - appetitive Konsequenz (positive Zweckmäßigkeit) oder eine anzustrebende Sicht seiner Selbst (positive Wesentlichkeit) vor Augen geführt wird. Während bei positiver Zweckmäßigkeit $(\mathrm{O}+)$ sprachlich explizit angenehme äußere und innere Folgen (external: «weil du so Anerkennung erntest»; internal: «weil du dich so gut fühlen wirst») dargeboten werden, wird mit positiver Wesentlichkeit (E+) das Sich-Lohnende lediglich impliziert: Das Befolgen der vom sozialen Umfeld herangetragenen normativen Erwartungen (external: «weil du das aus Rollenerwägungen musst»; internal: «weil du dich auf diese Weise sehen solltest») - so wird dem Kind signalisiert - führt mittelbar zu Anerkennung und Wohlbefinden. 
Mittels negativ formulierter Argumente (Code: «-») wird dem Kind eine veränderte Bedeutungszuschreibung hinsichtlich einer schulischen Aktivität oder eines Leistungsziels nahegelegt, indem ihm eine aversive Konsequenz vor Augen geführt wird, die es aus elterlicher Sicht zu meiden gilt. Bei negativer Zweckmäßigkeit sprachlich explizit $(\mathrm{O}-)$, bei negativer Wesentlichkeit Missachtung, Misserfolg und Leid wiederum lediglich insinuierend (E-), argumentieren die Eltern im Sinne von Geboten mit Kosten, die es zu vermeiden gelte, oder sprechen Verbote aus. Im Sinne eines negativen Verstärkers signalisieren sie dem Kind auf diese Weise die Alternativlosigkeit ihrer Bedeutungszuschreibung (negative Zweckmäßigkeit: «Es ist bedeutsam, dass du X tust/erreichst, weil du sonst eine schlechte Note haben wirst», «..., weil du dich sonst schlecht fühlen wirst»; negative Wesentlichkeit: «..., weil du das auf keinen Fall darfst», «..., weil du nicht jemand bist, der andere Möglichkeiten hat»).

Da die Eltern mit negativ formulierten Wertbegründungen die Akzeptanz ihres Kindes bezüglich der Richtigkeit oder Dringlichkeit ihrer Wert-Zuschreibung mit bedrohlichen, letztlich als zwingend dargestellten Gründen zu erreichen suchen, sind diese in motivational-affektiver Hinsicht als klar problematischer einzuschätzen als positiv formulierte Begründungen: Sofern das Kind die als positiv oder negativ präsentierten Argumente, wie von ihnen intendiert, tatsächlich als anziehend oder abschreckend interpretiert, dürften die Eltern mit ihren appetitiven Argumenten bei ihm positive Emotionen und mit ihren aversiven Argumenten negative Emotionen wecken (vgl. Frenzel \& Stephens, 2017, S. 39-40; Pekrun, 2006, S. 319-324). Im Zusammenspiel mit einer mittleren Kontrollzuschreibung des Kindes bezüglich der betreffenden Aktivität dürfte bei einer positiven Wertzuschreibung Hoffnung und Zuversicht ${ }^{25}$, bei einer negativen Wertzuschreibung dahingegen Angst und Zweifel ${ }^{26}$ mit ungünstigen Folgen für die Informationsverarbeitung, die (intrinsische) Motivation, den Strategieeinsatz sowie die Persistenz evoziert werden (vgl. Abschnitt 5.5.1.4).

\subsubsection{Die Dimension Lokus}

Die Dimension Lokus - die den inneren und äußeren Referenzbereich bezeichnet, auf den der Elternteil die Aufmerksamkeit des Kindes in seiner Begründung lenkt - dürfte hinsichtlich ihrer Effekte namentlich von den motivationalen Tendenzen

${ }^{25}$ Z.B.: «Meine Mutter sagt, dass es bedeutsam ist, dass ich täglich 20 Minuten übe, weil ich so bestimmt eine gute Note schreiben werde. Da es mir meist gelingt, mich selbständig aufzuraffen und an die Arbeit zu machen, kann ich auf ein gutes Gelingen hoffen.»

${ }^{26}$ Z.B.: «Meine Mutter sagt, dass es bedeutsam ist, dass ich täglich 20 Minuten übe, weil ich sonst den Übertritt nicht schaffe. Und da es mir manchmal schwerfällt mich aufzuraffen und an die Arbeit zu machen, fühle ich mich belastet und angespannt.» 
des jeweiligen Kindes abhängen. Kinder, die eine mastery goal orientation (vgl. Dweck, 1986; Nicholls, 1984) aufweisen und mit einer individuellen Bezugsnorm vornehmlich darauf bedacht sind, ihre Kompetenzen zu entwickeln und die Lerngegenstände gründlich zu verstehen (vgl. Abschnitt 4.2.1.2), dürften sich vermutlich von internalen Gründen stärker angesprochen fühlen («weil du so kognitiv, emotional und/oder behavioral profitierst» bzw. «weil du sonst negative kognitive, emotionale und/oder behaviorale Folgen zu gewärtigen hast»; «weil du jemand bist, der das kann» bzw. «weil du jemand bist, der das andere nicht kann»). Eine hohe Lernzielorientierung geht vermutlich aber nicht nur mit einer höheren Salienz von internalen Argumenten einher, sondern auch mit diesbezüglich intensiver erlebten positiven und negativen Emotionen (siehe oben). Umkehrt kann vermutet werden, dass Kinder, die eine performance goal orientation aufweisen und - an einer sozialen Bezugsnorm orientiert - primär darauf ausgerichtet sind, sich vor andern als kompetent und leistungsfähig zu erweisen (bzw. ihre scheinbaren Defizite nicht sichtbar zu machen), stärker auf externale Argumente in der elterlichen Begründung aufmerksam werden. Ebenso dürften sie bei solchen Argumenten Hoffnung und Zuversicht sowie Angst und Zweifel intensiver erleben («weil du so einen erwünschten sozialen Status erreichst»; «weil du das mit Blick auf soziale Normen einfach musst» bzw. «weil du sonst mit einem unerwünschten sozialen Status konfrontiert bist»).

Welches Zwischenfazit lässt sich bezüglich der Frage nach effektiven elterlichen Strategien auf der Grundlage der bisher erörterten Mechanismen der Dimensionen Valenz und Lokus ziehen? Eltern sind grundsätzlich gut beraten, wenn sie ihre Wertzuschreibungen generell - im Sinne eines Argumentationsstils - mit positiven internalen Begründungen (Oi+/Ei+) zu unterstreichen suchen. Solche Begründungsmuster locken das Kind sprachlich mehr oder weniger explizit mit den angenehmen Folgen der angesprochenen Aktivität oder des Ziels, evozieren positive Emotionen, die bezüglich der Beachtung und Befolgung des Gesagten motivational günstig sein dürften, und lenken im Sinne der mastery goal orientation und eines incremental mindsets (vgl. Abschnitt 4.2.1.2) durch den Verweis auf innere Aspekte des Kindes dessen Blick auf seine persönlichen Bedürfnisse bzw. auf die Entwicklung seiner selbst- und sachbezogenen Kompetenzen. Zwar scheint es situativ - z. B. bei einer Überschätzung und Sich-in-falscher-SicherheitWiegens des Kindes - durchaus angemessen und notwendig, mittels negativen, auf Gefahren und gesellschaftliche Restriktionen hinweisenden Argumenten die Dringlichkeit eines Beachtens der Bedeutungszuschreibung unmittelbar deutlich zu machen (z. B. «Merke dir: es ist bedeutsam, dass du dich wegen deiner sehr guten Note heute in Französisch nur kurz zurücklehnst und heute Abend trotzdem Englischwörter lernst, weil du sonst morgen eine ungenügende 
Note im Vokabeltest haben wirst.»). Doch dürften die negativen Begründungstypen $(\mathrm{Oe}-/ \mathrm{Oi}-/ \mathrm{Ee}-/ \mathrm{Ei}-)$ wegen ihres angsterzeugenden Potentials und den zumindest langfristig damit einhergehenden negativen Folgen auf das psychische Wohlbefinden und das Handeln ungünstige Strategien der verbalen Wertvermittlung darstellen. Kinder mit einer ausgeprägten Wettbewerbsorientierung bzw. einer performance goal orientation dürften sich sodann von positiven externalen Argumenten $(\mathrm{Oe}+/ \mathrm{Ee}+)$ besonders angesprochen fühlen und der solcherlei herausgestrichenen Bedeutsamkeit eines bestimmten Handelns denn wohl auch in erhöhtem Maß Beachtung schenken. So sinnvoll der Einsatz dieser Strategien situativ ist, so problematisch dürfte es sein, wenn diese Argumentationsstrategie das dominante Stilelement der elterlichen Wertvermittlung darstellt: Insofern als die Eltern dauernd auf Belohnung durch soziale Anerkennung und gesellschaftliche Teilhabe verweisen, implizieren sie gleichzeitig aber auch immer wieder die autonomieeinschränkende Notwendigkeit, den Erwartungen anderer genügen zu müssen, und betonen den Wettbewerbscharakter von Lern- und Leistungssituationen, was im Zusammenspiel mit geringen entsprechenden Kontrollüberzeugungen zu Druck, Frustration und Hoffnungslosigkeit führen kann (vgl.Frenzel \& Stephens, 2017, S. 41-42).

\subsubsection{Die Dimension Aufgabenwert}

Es stellt sich nun die Frage, welche Effekte von der Dimension Aufgabenwert, also der eigentlichen wertbezogenen Botschaft in den beiden Ausprägungen Zweckmäßigkeit (O) und Wesentlichkeit (E), auf Aufmerksamkeits-, Verarbeitungs- sowie motivational-affektive Prozesse beim Kind ausgehen: Unter welchen Bedingungen steigt die Wahrscheinlichkeit, dass das Kind den einer bestimmten schulischen Aktivität zugeschriebenen task value nicht nur zur Kenntnis nimmt, sondern in sein Überzeugungssystem integriert?

Argumentieren die Eltern mit Zweckmäßigkeit (..., weil du so etwas Erwünschtes erreichst bzw. «..., weil du so etwas Unerwünschtes vermeidest»), bringen sie den utility value zum Ausdruck, den sie persönlich der Aktivität bzw. dem Ziel zuschreiben. Begründen sie dahingegen mit der Wesentlichkeit eines bestimmten Handelns, signalisieren sie dem Kind, dass sie demselben einen attainment value und somit eine hohe Relevanz («..., weil du das musst» bzw. «..., weil du das andere auf keinen Fall darfst») beimessen. Die Wichtigkeit ergibt sich aus dem Umstand, dass ein Bezug zum Selbst bzw. zur persönlichen oder sozialen Identität des Kindes (vgl. Eccles, 2005, S. 109) hergestellt wird (vgl. Abschnitt 5.5.1.2). Auf Anhieb wird damit deutlich, dass bei einer Begründung mit Zweckmäßigkeit der Grad an Selbstbestimmung, der dem Kind zugestanden wird, grundsätzlich höher ausfällt und von diesem entsprechend als 
autonomiegewährendes Vorgehen (vgl. Abschnitt 2.2.2.4, insb. Tabelle 2.1) interpretiert werden dürfte. Wesentlichkeitsaussagen haben es dahingegen an sich, dass sie die Unumgänglichkeit eines Verhaltens signalisieren und mit Blick auf die Autonomiebedürfnisse des Kindes einen beschneidenden und somit problematischen Charakter aufweisen. Inwiefern das Kind hierbei intrusive Kontrolle (coersion) (vgl. Grolnick \& Ryan, 1989; Skinner, E. A. et al., 2009) wahrnimmt (vgl. Abschnitt 2.2.2.4) und welche Reaktion es darauf zeigt, dürfte vor allem davon abhängen, inwieweit es die diesbezügliche Bedeutungseinschätzung bereits teilt, wie seine diesbezüglichen Kontrollüberzeugungen beschaffen sind und in welchem kommunikativen Modus die Bedeutungszuschreibung vermittelt wird.

Wie in Abschnitt 5.5.1.3 mit den berichteten belgischen Interventionsstudien von Simons et al. $(2003,2004)$ bereits deutlich wurde, steht in den bisherigen empirischen Untersuchungen zur verbalen Beeinflussung der Relevanz von schulischen Aktivitäten nahezu ausschließlich der utility value - also der Aspekt der Nützlichkeit - im Zentrum. Studien dazu, wie Lehrkräfte und Eltern verbal den attainment value, den intrinsic value und die Kostenüberlegungen von Kindern und Jugendlichen beeinflussen, fehlen bisher weitgehend (vgl. Lazarides et al., 2015, S. 54; Wigfield, Rosenzweig \& Eccles, 2017, S. 126). Neben dem Umstand, dass sich der selbstperzipierte utility value in etlichen Studien nicht nur bezüglich des Interesses, des Engagements und der Persistenz, sondern ebenso bezüglich Leistungsergebnissen von Schülerinnen und Schülern als prädiktiv erwiesen hat (vgl. Abschnitt 5.5.1.3), dürfte die Fokussierung auf diese Facette auch dem oben angesprochenen didaktischen Prinzip des Lebensweltbezugs geschuldet sein, von dem man sich u. a. eine motivationsförderliche Funktion bezüglich der Unterrichtsinhalte verspricht. Nicht selten dürften Lehrkräfte die Schülerinnen und Schüler sprachlich-explizit auf die Nützlichkeit von Inhalten und Verfahren für deren Alltag aufmerksam machen. So berichten Hulleman und Barron (2016), die zusammen mit Harackiewicz und Mitarbeitenden in den letzten Jahren die bedeutendsten Untersuchungen zu den Gelingensbedingungen einer erfolgreichen Beeinflussung des utility value von Lernenden durchgeführt haben (vgl. Canning \& Harackiewicz, 2015; Harackiewicz, Rozek, Hulleman \& Hyde, 2012; Hulleman et al., 2010; Hulleman \& Harackiewicz, 2009), wie ihre Erfahrungen als Dozierende von Statistikkursen für die Planung ihrer ersten diesbezüglichen Interventionsstudien leitend waren:

Faced with the challenge of students who lack value for statistics, an obvious way was to help students discover how the statistical techniques they were learning applied to their lives in some way (utility value). This seemed more plausible than convincing 
students that learning statistics was fun (intrinsic value) or an important part of their identity (attainment value). (Hulleman \& Barron, 2016, S. 166)

Die Grundidee, die hier wohl nicht zuletzt vor dem Hintergrund der Selbstbestimmungstheorie, genauer der Organismic Integration Theory (vgl. Deci \& Ryan, 1985; Ryan \& Deci, 2002, 2016) (vgl. Abschnitt 5.5.1.2), durchscheint, dürfte darin bestehen, Menschen primär die Nützlichkeit einer Aktivität bzw. eines Ziels für ihre größeren Ziele verständlich zu machen und darauf zu zählen, dass mit den Erfolgserfahrungen und den damit steigenden Kompetenz- und Selbstwirksamkeitsüberzeugungen - bzw. mit zunehmendem Erleben eines hohen Grades von Eigenkompetenz und Selbstbestimmtheit (vgl. Ryan \& Deci, 2016) - sich persönliche Wichtigkeit und Interesse beim Individuum selbstgeneriert einstellen.

Auf den folgenden Seiten werden drei experimentelle Interventionsstudien eingehender erörtert, mit denen Canning und Harackiewicz (2015) mehr Licht in die Frage zu bringen vermochten, welche Bedingungen direkt kommunizierte Zweckmäßigkeitsaussagen aufweisen müssen, damit sie sowohl bei Lernenden mit tiefen als auch bei solchen mit hohen Kontrollüberzeugungen (high confidence vs. low confidence students) zu einer Erhöhung ihres utility value in der betreffenden Domäne führen. Aus den Befunden der drei aufeinander aufbauenden Laborstudien sollen in der Folge Gestaltungsprinzipien für Zweckmäßigkeitsargumente herausgearbeitet und auf deren Basis schließlich auch solche für Wesentlichkeitsargumente abgeleitet werden.

In Studie 1 wurde 88 College-Studierenden verschiedener Fachrichtungen in einer Powerpoint-Präsentation eine neuartige mentale Technik zum Lösen komplexer Multiplikationsprobleme vermittelt, welche sie im Anschluss während drei Minuten einüben konnten. Diejenigen Studierenden, die nach dem Zufallsprinzip der Gruppe «direkt kommunizierter utility value» zugeteilt worden waren, erfuhren in der Präsentation zusätzlich, welchen Nutzen ihnen die Technik in Alltagshandlungen wie beispielsweise dem Einkaufen sowie in zukünftigen Tätigkeiten im Rahmen ihrer beruflichen Karriere bringen könne. Die Studierenden der Gruppe «selbstgenerierter utility value» wurden aufgefordert, sich selber diesbezügliche Beispiele zu überlegen und die Relevanz nach dem Üben in einem kleinen Essay zu beschreiben ${ }^{27}$. Die Studierenden der Kontrollgruppe sahen nur

\footnotetext{
${ }^{27}$ Es handelt sich dabei um eine auf Partizipation und Personalisierung zielende Interventionstechnik, die zu den sog. saying-is-believing- oder self-persuasion-Methoden gezählt werden kann (vgl. Canning \& Harackiewicz, 2015, S. 48). Im Kern werden Individuen bei diesen Methoden dazu ermutigt, die von der Intervention anvisierte Botschaft anderen oder sich selbst auf der Grundlage eigener Erfahrungen zu erörtern und so einen Teil der Regulation selbst zu übernehmen. Durch die erhöhte personale Passung der Botschaft erhofft man sich
} 
die Präsentation der mathematischen Prozedur. Alle Teilnehmenden waren nach Abschluss der Einführungs- und Übungsphase (aber vor der Schreibaufgabe, vgl. Studie 2 unten) aufgefordert, in einem Fragebogen ihre Erfolgszuversicht einzuschätzen und danach zur Ermittlung der task performance sechzig Multiplikationsprobleme mit der neuen Technik zu lösen. Am Ende des Experiments wurden sie aufgefordert, in einem Fragebogen Auskunft über den von ihnen wahrgenommenen utility value - z. B. «This technique could be useful to me in my future career», «This technique could be useful to me in my daily life» - sowie über ihr situationales Interesse - u. a. «Using this multiplication technique is fun»-Auskunft zu geben (Canning \& Harackiewicz, 2015, S. 53). Im Vergleich mit der Kontrollgruppe zeigte sich, dass bei den Studierenden mit hohen Kontrollüberzeugungen beide Interventionstechniken einen signifikanten Effekt auf den wahrgenommenen utility value hatten, wohingegen bei denjenigen mit tiefen Kontrollüberzeugungen beide Techniken zu keiner erhöhten Nützlichkeitseinschätzung führten. Die Autorinnen vermuten, dass weniger zuversichtliche Individuen die wertbezogene Information nicht aufnehmen könnten, wenn sie gedanklich mit Zweifeln an den eigenen Fähigkeiten beschäftigt seien: «It may be that for less confident individuals to internalize the utility of the math technique, they first need to believe they can succeed at the task» (Canning \& Harackiewicz, 2015, S. 56).

Beim direkten Vergleich der beiden Interventionstechniken zeigten sich sodann bei den Studierenden mit geringer Kontrollüberzeugung sehr unterschiedliche Effekte hinsichtlich der Leistung und des Interesses: Anders als bei den selbstgenerierten Nützlichkeitseinschätzungen gingen die direkt kommunizierten Zuschreibungen bei ihnen mit schwächeren Leistungsergebnissen und geringerem Interesse einher: «Not only was directly communicated [utility value] information not helpful for participants with lower confidence, it actually seemed to lead

eine größere Überzeugungskraft derselben: «It encourages recipients to author the intervention message and to view their experience through its lens without feeling controlling or stigmatizing» (Walton, 2014, S. 80). Hulleman et al. (2010) konnten experimentell zeigen, dass es den Universitätsstudierenden der Interventionsgruppe, die den Auftrag hatten, laufend die selbstperzipierte Nützlichkeit der Studieninhalte «für ihr Leben» in einem Logbuch zu formulieren, konsistent besser gelang, ihr Anfangsinteresse über das Semester zu bewahren als den Studierenden der Kontrollgruppe. Ebenso bekundeten sie nach den Treatments ein größeres Interesse daran, ihr diesbezügliches Wissen weiter zu vertiefen. In Feldstudien auf der Sekundarstufe I (vgl. Hulleman \& Harackiewicz, 2009) zeigte sich ferner, dass vor allem die Schülerinnen und Schüler mit tiefen Erfolgserwartungen von dieser schriftlichen utility-value-Intervention profitierten, indem sie zum zweiten Messzeitpunkt über signifikant höhere Leistungszuwächse und Interessewerte bezüglich der im Treatment fokussierten Naturwissenschaften verfügten als die low-expectancy-Lernenden der Kontrollgruppe. 
these participants to react negatively to the information» (Canning \& Harackiewicz, 2015, S. 56). Die Autorinnen vermuten, dass die Fremdregulation bei den wenig zuversichtlichen Studierenden blockierend auf den Lernprozess und die Interessenentwicklung eingewirkt haben könnte, weil sie von diesen mit zu hohen Erwartungen bzw. Anspruchsniveaus von außen und somit als Druck interpretiert worden sei: «Information about the utility of the technique coupled with a lack of confidence may put too much pressure on these individuals, without giving them a chance to process or cope with the [utility value] information by putting it in their own words» (Canning \& Harackiewicz, 2015, S. 56).

Die schriftliche Wertbestimmung aus der eigenen Perspektive hat sich dahingegen für alle Studierenden der Interventionsgruppe «selbstgenerierter utility value», namentlich aber für diejenigen mit geringen Kontrollüberzeugungen, als förderlich bezüglich der Leistung erwiesen. Wie die Autorinnen meinen, könnte es sein, dass das Vorstellen und Beschreiben von zukünftigen Situationen, in denen die Aktivität nützlich ist - sich das Individuum vor dem inneren Auge also als jemand sieht, der zukünftig, nach einer Phase des Übens über die Technik verfügt und diese selbstgesteuert einsetzt - den Druck etwas mindert, kurzfristig unbedingt gut abschneiden zu müssen. Diese unbeschwertere Sichtweise scheint die Leistungserbringung durch einen stärkeren Fokus auf den Explorations- und Übungsaspekt («ich probiere es halt mal») befördert zu haben (vgl. Canning \& Harackiewicz, 2015, S. 57). Notabene hatten auch die Untersuchungsleitenden die Instruktion für das Kurzessay so formuliert, dass die Teilnehmenden über ihre aktuell noch geringen Fähigkeiten im Umgang mit der Prozedur leichter hinwegsehen und den Blick für die Nützlichkeitsbeurteilung auf die Zukunft werfen konnten, wenn sie einen höheren Fähigkeitsgrad erreicht haben würden (man beachte im folgenden Zitat den Verständnis signalisierenden Einsatz von «of course»):

Type a short essay (1 to 3 paragraphs in length) briefly describing the potential relevance of this technique to your own life. Of course, you'll probably need more practice with the technique to really appreciate its personal relevance, but for purposes of this short essay, please focus on how this technique could be useful to you in your own life, and give examples. (Canning \& Harackiewicz, 2015, S. 52, Hervorhebung E.S.)

In Anbetracht des starken Effekts des selbst generierten utility values auf das Leistungsergebnis bei allen Studierenden dieser Interventionsgruppe erscheint es auf den ersten Augenblick sonderbar, dass die Maßnahme aber bei denjenigen mit geringer Kontrollüberzeugung zu keinem Effekt bezüglich deren perzipierten utility value und deren situationalem Interesse geführt hat. Canning und Harackiewicz mutmaßen, dass die Hauptursache hierfür darin liegen könnte, dass sich 
die wenig zuversichtlichen Studierenden während der Einführung in die Multiplikationstechnik den Nutzen derselben schlicht zu schlecht hätten vorstellen können:

Perhaps students first need to hear about some examples of utility value from someone else and then be given the chance to come up with their own personalized examples. Directly communicated UV [utility value] information might be too threatening by itself for individuals who lack confidence [...], but once these individuals are given the chance to process the information in their own words, directly communicated UV might actually be helpful. (Canning \& Harackiewicz, 2015, S. 57)

In Studie 2 prüften die Forscherinnen deshalb, ob eine Kombination der beiden Interventionsstrategien einen synergetischen Effekt auf den perzipierten utility value, das Interesse und die Leistung entfalte und ob eine solchermaßen kombinierte Interventionsstrategie tatsächlich für die Gruppe der wenig Zuversichtlichen am effektivsten sei. Die 113 teilnehmenden Studierenden wurden dazu nach dem Zufallsprinzip einer der folgenden vier Gruppen zugewiesen: «kombinierte utility value-Intervention», «nur direkt kommunizierter utility value», «nur selbstgenerierter utility value» sowie «weder das eine noch das andere» (Kontrollgruppe). Die Studierenden der kombinierten Intervention erhielten im Rahmen der Powerpoint-Präsentation die gleichen Nützlichkeitsinformationen wie die Gruppe «nur direkt kommunizierter utility value» und schrieben danach mit der gleichen Instruktion ein Kurzessay wie die Gruppe «nur selbstgenerierter utility value». Um zu untersuchen, ob Veränderungen bei den Kontrollüberzeugungen für die Effekte der utility value-Interventionen (mit)verantwortlich sein könnten, wurden diese nicht nur nach der Powerpoint-Präsentation mit den direkt kommunizierten Nützlichkeitsinformationen für den Alltag und die Karriere, sondern auch nach dem Schreiben der selbst-generierten Nutzenszenarien unmittelbar vor den Leistungsaufgaben erhoben.

Die kombinierte Strategie hat sich in den Analysen unabhängig von den ursprünglichen Kontrollüberzeugungen für alle Studierenden als kontrollförderlich erwiesen. Wie sich in der Prozessanalyse gezeigt hat, hat jeder Interventionsschritt einen eigentlichen «boost in perceived confidence» (Canning \& Harackiewicz, 2015, S. 61) bewirkt. Besonders bedeutsam wirkte sich dies für diejenigen Studierenden aus, die ursprünglich über geringe Kontrollüberzeugungen verfügt haben. Während Studie 1 gezeigt hat, dass bei ihnen unabhängig von der eingesetzten Interventionsstrategie kein erhöhter utility value für die Multiplikationstechnik erzielt werden konnte und die Interessenentwicklung und Leistungserbringung durch alleiniges Direktvermitteln von Zweckmäßigkeit 
offenbar behindert wurden, konnten durch die Mediation der gesteigerten Kontrolleinschätzungen nun sowohl der utility value, das Interesse als auch die Leistungsergebnisse signifikant erhöht werden:

Perhaps participants who are initially unsure of their ability can benefit from directly communicated UV [utility value] information, but need the opportunity to reflect on what they've learned. Giving these participants a chance to generate their own personal examples of UV after receiving some UV information from an external source might help them digest the material and put it in their own words. (Canning \& Harackiewicz, 2015, S. 61)

Offenbar hatte namentlich für die Wenig-Zuversichtlichen a) die direkte Vermittlung der Nützlichkeit eine informative Funktion für die Genese eigener Nützlichkeitsvorstellungen und b) die Aufforderung, eigene Nützlichkeitsvorstellungen in Sprache zu fassen, eine angst- und druckmindernde Funktion. Dies ließ sich auch anhand der Inhaltsanalysen belegen, die an den Essays der Studierenden durchgeführt wurden (vgl. Canning \& Harackiewicz, 2015, S. 60). U.a. zeigte sich hierbei, dass alle Studierenden signifikant seltener Wörter benutzten, die Besorgnis ausdrücken (z. B. to struggle with, to doubt), wenn sie die kombinierte Intervention erlebt hatten. Ferner offenbarten die Inhaltsanalysen auch, warum die kombinierte Strategie gerade bei den Studierenden mit geringen Kontrollüberzeugungen so erfolgreich gewesen sein mag: «This combination seemed to help participants who lacked confidence to write more about leisure activities such as shopping and eating out in restaurants, rather than how mental math can be useful in their career» (Canning \& Harackiewicz, 2015, S. 62). Möglicherweise fühlten sich diese Studierenden also spezifisch durch die Karrierebeispiele in den direkt kommunizierten Nützlichkeitsbotschaften bedroht.

Um dies zu prüfen, wurden die 134 Teilnehmenden der Studie 3 zufällig zu einer der drei folgenden Bedingungsgruppen zugeteilt: «direkt kommunizierter utility value nur mit Alltags-/Freizeitbeispielen», «direkt kommunizierter utility value mit Alltags-/Freizeit- und Karrierebeispielen» sowie «weder das eine noch das andere» (Kontrollgruppe). Bei der ersten Gruppe wurden mit anderen Worten in der Powerpoint-Präsentation zur Einführung in die Multiplikationsmethode karriere- und studiumsbezogene Beispielbilder sowie entsprechende Nützlichkeitsaussagen - z. B. «most college graduates enter professions that require math, so mental math can be useful in your future career» (Canning \& Harackiewicz, 2015, S. 62) - entfernt. Die verbleibenden Nützlichkeitsaussagen zur Multiplikationstechnik bezogen sich auf aktuelle Freizeitaktivitäten wie Banktransaktionen, das Einkaufen, Trinkgelder geben, aber auch auf zukünftige Situationen wie die Budgetplanung der Familie oder das Tätigen von finanziellen Investments. 
Tatsächlich ließ sich durch die Entfernung karriere- und studiumbezogener Beispiele und durch die alleinige Betonung von Alltagsbeispielen aus dem privaten Kontext das situationale Interesse und die Wahrnehmung von utility value bei denjenigen Studierenden, die ursprünglich an ihren diesbezüglichen Fähigkeiten zweifelten, in Relation zu denjenigen der Gruppe «direkt kommunizierter utility value mit Alltags-/Freizeit- und Karrierebeispielen» erhöhen - wiederum vermittelt über gesteigerte Kontrolleinschätzungen. Die anfänglich bereits Zuversichtlichen berichteten bei einer Fokussierung auf Alltagsbeispiele über einen höheren utility value, bezogen auf das Interesse hatten die unterschiedlichen Informationen bei ihnen keinen Effekt. Das Experiment zeitigte sodann keinerlei bedeutsamen Effekte auf die Leistungsergebnisse.

Wie Canning und Harackiewicz (2015, S. 65) schreiben, zeige sich, dass Lernende mit geringen Kontrollüberzeugungen für diejenigen Nützlichkeitsaussagen zur Multiplikationstechnik empfänglich seien, welche sich auf Aktivitäten bezögen, die gut in ihr Leben integriert seien und in ihrer Alltäglichkeit als besonders realistisch, aber auch bewältigbar erschienen. Das Studium und die zukünftige Karriere bildeten dahingegen Kontexte, die einerseits durch die druckerzeugenden Erwartungen und Vorgaben anderer geprägt seien, andererseits - je nach konkretem Studienfach -aber auch schlicht einen zu geringen Bezug zur mathematischen Technik hätten aufkommen lassen:

In sum, it isn't the case that directly communicated relevance is always threatening for less confident individuals, but rather that [utility value] information needs to be tailored to the characteristics and needs of the individual, and accommodate those who have trouble imagining themselves succeeding at the task. (Canning \& Harackiewicz, 2015, S. 65)

Trotz der zahlreichen Limitationen dieser drei experimentellen Studien ${ }^{28}$ stellen sie angesichts des Fehlens einer breiten Befundlage einen wichtigen Schritt zur Klärung der Frage dar, unter welchen Bedingungen die Wahrscheinlichkeit steigt, dass verbale Bedeutsamkeitszuschreibungen von Dritten bei verschiedenen Rezipient*innen die intendierte Wirkung zu entfalten vermögen.

\footnotetext{
${ }^{28}$ Begrenzungen der Aussagekraft ergeben sich vor allem aus der Spezifität des Inhalts, auf den sich die Nützlichkeitsregulationen beziehen, aus der geringen Dauer der Interventionen (einmalig, ca. eine Stunde dauernd), aus der fehlenden Gleichzeitigkeit bzw. veränderten Reihenfolge der miteinander verglichenen Interventionen (die direkten utility value-Interventionen fanden stets vor den selbstgenerierten statt), aus dem Alter sowie dem lediglich rudimentär berücksichtigten Lebenskontext der Teilnehmenden (vgl. Canning \& Harackiewicz, 2015, S. 66-68).
} 
Damit elterlichen Wertregulationen, die auf Zweckmäßigkeitsargumenten beruhen (..., weil du so etwas Erwünschtes erreichst bzw. «..., weil du so etwas Unerwünschtes vermeidest»), vom Kind Aufmerksamkeit geschenkt wird und sich Änderungen in seinem utility value betreffend der entsprechenden schulischen Aktivitäten und Ziele ergeben, so lässt sich aus den Studien schließen, dürften die folgenden Bedingungen relevant sein:

a) Das Kind muss dem von den Eltern präsentierten Zweck selbst eine hohe Bedeutung beimessen

Wie die Interventionsstudien von Canning und Harackiewicz (2015), aber auch von Simons et al. $(2003,2004)$ gezeigt haben, müssen die Kinder grundsätzlich den Zweck - also das, was die Eltern als «Oberziel» (Esser, 2001, S. 263) deklarieren («...weil du damit Y erreichst») - auch selber tatsächlich als wertvolles Ziel erkennen ( $« \mathrm{Ja}, \mathrm{Y}$ ist mir wichtig»). Je wichtiger dem Kind dieses Ziel ist, desto mehr Bedeutung dürfte es der von den Eltern in ihrem Appell als Mittel präsentierten schulbezogenen Aktivität (Merke dir: Es ist bedeutsam, dass du X tust») zuschreiben. Oder umgekehrt: Je gleichgültiger ihm dieser Zweck ist, desto weniger Beachtung und Engagement wird das Kind dem von den Eltern als Mittel dargelegten Handeln bzw. Ziel zukommen lassen und desto weniger wird es die Bedeutsamkeitseinschätzung bezüglich des betreffenden Handelns verändern ( $\mathrm{X}$ ist für mich nicht bedeutsam, da ich Y nicht will»). Um mit einer Zuschreibung von Zweckmäßigkeit ihr Ziel einer veränderten Wertzuschreibung bezüglich eines bestimmten schulbezogenen Handelns (und subsequent ein verändertes diesbezügliches Handeln) zu erreichen, ist es für die Eltern mit anderen Worten wichtig, die eigentlichen - essenziellen - Ziele und Werte des Kindes zu kennen. Lassen sie es hierin an Passung fehlen, dürfte die Argumentationsstrategie der Zweckmäßigkeit ins Leere laufen.

b) Das Kind muss die Zweckmäßigkeitsaussage für glaubwürdig halten

Ebenso muss wohl die von den Eltern deklarierte Mittel-Zweck-Relation selber glaubwürdig sein. So dürften sich in den Studien 2 und 3 von Canning und Harackiewicz (2015) etliche Probandinnen und Probanden nicht von jenen direkten utility value-Botschaften angesprochen gefühlt haben, die einen Bezug zur zukünftigen Karriere herstellten, weil sie die deklarierte Nützlichkeit der mathematischen Prozedur für ihre Karriere oder ihr Studium schlichtweg bezweifelten. Eltern müssen demnach um realistische Argumentationen bemüht sein. 
c) Das Kind sollte über positive Kontrollüberzeugungen bezüglich der angesprochenen Aktivität sowie bezüglich des in der Begründung genannten Ziels verfügen

Im Einklang mit dem Postulat der Expectancy-Value-Theorie (vgl. Abschnitt 5.2), wonach task values das Denken und Handeln dann am stärksten beeinflussen, wenn auch die Erfolgserwartung bzw. die Kontrollüberzeugungen hoch sind (vgl. Jacobs \& Eccles, 2000, S. 413), lässt sich aus den Befunden von Canning und Harackiewicz (2015) ableiten, dass die Eltern mit ihren Zweckmäßigkeitsaussagen grundsätzlich dann eine veränderte Wertzuschreibung und entsprechendes Interesse und Engagement herbeiführen können, wenn das Kind bezogen auf das im Appell ins Zentrum gestellte Mittel als auch bezogen auf den Zweck über positive Kontrolleinschätzungen verfügt. Wie die Ergebnisse der Studien 2 und 3 von Canning und Harackiewicz (2015) nahelegen, dürfte der angeführte Zweck (bedeutsam für die zukünftige Karriere) und der Umstand, dass es sich beim Mittel um eine mathematische Aufgabe handelte, je als Einzelfaktoren oder aber im Zusammenspiel bei etlichen Studierenden kompetenzbezogene Zweifel und damit einhergehend Ängste bzw. Druckempfinden ausgelöst haben. Es ist mit anderen Worten wichtig, dass Eltern es auch hierin nicht an Passung fehlen lassen: Sie sollten bei verbalen Wertregulationen mit der Zweckmäßigkeitsstrategie darauf achten, dass sich ihr Kind weder bezüglich des Mittels noch bezüglich des Zwecks überfordert und folglich zu stark unter Druck fühlt.

d) Das Kind sollte stets Möglichkeiten erhalten, Stellung zu den präsentierten Nützlichkeitsargumenten zu nehmen und ggf. selber plausiblere, noch attraktivere und weniger belastende Nutzenszenarien formulieren können

Die bisherigen Folgerungen für die Gestaltung wirksamer Zweckmäßigkeitszuschreibungen zeigen, wie wichtig es ist, dass die verbalen Ansprachen der Eltern sich gut in die bisherigen Kontroll- und Wertüberzeugungen des Kindes einpassen. Jacobs und Eccles (2000) streichen die fundamentale Bedeutung dieses Prinzips für die Regulation aller task values heraus:

[...] children are unlikely to begin to value activities that do not match either their social identities or their personal identities. Similarly, they are unlikely to develop task values in contexts in which they feel incompetent, have no control, or feel unsupported. (Jacobs \& Eccles, 2000, S. 411) 
Der von Canning und Harackiewicz (2015) in Studie 2 berichtete eindrückliche Effekt der Kombination aus direkt kommuniziertem utility value und selbst formulierten utility value-Beispielen auf die Werteinschätzung, das Interesse und die Leistung bei allen Probandinnen und Probanden, namentlich aber bei den wenig zuversichtlichen, weist den Weg, wie das weiter oben von Canning und Harackiewicz (2015, S. 65) geforderte «tailor[ing] to the characteristics and needs of the individual» bewerkstelligt werden sollte: Wichtig ist, dass Eltern einen dialogisch-kokonstruktiven Ansatz (vgl. Abschnitt 2.2.2.3) wählen und dem Kind die Möglichkeit geben, auf ihre zweckbezogenen Argumente einzugehen, Widerspruch anzumelden, im Sinne eines kontingenten Scaffoldings (vgl. Belland, 2014; Reiser \& Tabak, 2014; Wood et al., 1976) stichhaltigere und passendere Argumente bei ihnen anzufordern und zu erhalten und - von den Eltern explizit dazu ermutigt oder aber im Zuge der Diskussion dazu gedrängt - selbst Nutzenbeispiele zu formulieren und/oder ihre Befürchtungen zum Ausdruck zu bringen. Der Blick weitet sich dabei von der isolierten Betrachtung des elterlichen Regulations-Moves (ausgedrückt im Frame) zur ganzen Handlungsepisode: Die mehrfach angemahnte Passung von Wertregulation mit Nützlichkeitsargumenten hinsichtlich des Zwecks, des Mittels und der Kontrollüberzeugung verlangt von den Eltern ein Bemühen um einen dialogischen Kommunikationsmodus der grundsätzlich von gegenseitigem Argumentieren, Klären und Erklären geprägt ist. Eltern treten beim Motivieren durch Zweckrationalitäten als Beratende auf und dürften umso erfolgreicher in ihrem cognitive structuring ${ }^{29}$ (vgl. Abschnitt 2.2.2.4, insb. Tabelle 2.1) sein, je besser ihnen durch ein dialogisches Vorgehen die Diagnose sowie der Anschluss an die Sprache, die Sichtweisen, Ziele und Kontrollbedürfnisse des Kindes gelingt.

An dieser Stelle fragt sich mit Blick auf die genannten Gestaltungsprinzipien, welches die Charakteristika von Wertzuschreibungen sind, bei denen die Eltern mit der Wesentlichkeit einer Aktivität bzw. eines Ziels argumentieren, wie diese auf das Kind wirken und wie diese Effekte durch Gestaltungsmaßnahmen allenfalls optimiert werden könnten. Wie oben erwähnt, beschränkt sich die relativ geringe Zahl an Experimenten zu verbalen Wertregulationen bisher auf utility value-bezogene Interventionen (vgl. Lazarides et al., 2015, S. 54; Wigfield et al.,

\footnotetext{
${ }^{29}$ Tharp und Gallimore (1988, S. 63): «[...] 'Cognitive structuring' refers to the provision of a structure for thinking and acting. It may be a structure for beliefs, for mental operations, or for understanding. It is an organizing structure that evaluates, groups and sequences perception, memory, and actions. In science, it is theory; in religion, it is theology; in games, it is rules. In everyday life, cognitive structures are like all of these, more or less formalized, more or less conscious.»
} 
2017, S. 126), so dass die folgenden Überlegungen in noch höherem Maß den Status von Hypothesen beanspruchen müssen.

Während Bedeutsamkeitszuschreibungen mit Zweckmäßigkeitsargumenten als Ratschlag verstanden werden können, den man grundsätzlich befolgen kann oder auch nicht, bergen Wesentlichkeitsargumente, wie bereits vorgebracht, eine weit bindendere Botschaft an das Kind: Mit Wesentlichkeitsargumenten werden die angesprochenen schulischen Aktivitäten und Ziele zur erwarteten Norm oder Pflicht erhoben. Dies wird besonders deutlich bei Wertregulationen mit externaler Wesentlichkeit (Ee), in denen Rollenerwartungen (vgl. Abschnitt 2.1) zum Ausdruck gebracht werden (z. B. «Es ist bedeutsam, dass du X tust, weil du das als angehende Oberstufenschülerin einfach musst» bzw. «weil du als Mädchen etwas anderes auf keinen Fall tun darfst»), ist aber ebenso ersichtlich, wenn mit internaler Wesentlichkeit (Ei) operiert und wertrational mit Bezug auf das personale Selbst argumentiert wird (z. B. «Es ist bedeutsam, dass du X tust, weil du (nicht) das Potential hast, es in die Abteilung A zu schaffen»). Die Unumgänglichkeit, Zwangsläufigkeit und Alternativlosigkeit, die zum Ausdruck gebracht wird, schränkt den Entscheidungs- und Handlungsspielraum des Kindes ein («Meine Mutter verlangt das von mir. Sie meint, dass es sich für mich so gehört»). Inwiefern dies von diesem als intrusive Kontrolle (coersion) (vgl. Grolnick \& Ryan, 1989; Skinner, E. A. et al., 2009) interpretiert wird und welche äußere Reaktion es darauf zeigt (überzeugte vs. vordergründig-suggerierende Zustimmung, offene vs. verdeckte Ablehnung), dürfte in noch weit höherem Maß als bei ZweckmäBigkeitsargumenten davon abhängen, inwieweit es die signalisierte hohe Relevanz bereits teilt («Ist das wirklich so wichtig?»), wie hoch das Kind seine Kontrolle bezüglich des als essentiell charakterisierten Handlungsziels veranschlagt ( Habe ich das, was meine Mutter als sehr wichtig bezeichnet, im Griff?»), aber auch in welchem kommunikativen Modus die Bedeutungszuschreibung vermittelt wird (vgl. Abschnitt 5.7).

Wesentlichkeitsargumente dürften wegen ihrer zugespitzten und auf das Kind bezogenen Aussage («weil du musst», «weil du nicht darfst», «weil du das doch (nicht) kannst») grundsätzlich eine hohe Salienz aufweisen. Gekoppelt mit einer prägnanten Darbietung des Appells (z. B. «Achtung! Es ist bedeutsam, dass du...») scheinen sie denn auch besonders angemessen zu sein, wenn es darum geht, die Aufmerksamkeit des Kindes auf Gefahren oder Fehler mit drastischen Folgen zu lenken, die ihm nicht bewusst zu sein scheinen. Je zutreffender dies aus der Sicht des Kindes ist («es war mir nicht bewusst»), je leichter es sich das Zwanghafte dieser Situationen erklären kann - und ihm gegenüber bestenfalls möglichst dialogisch auch geklärt wird («ich sehe es ein») -, je sporadischer und situationsangemessener sie eingesetzt werden («wenn meine Mutter sagt, es 
sei wichtig für mich, dann trifft dies in der Regel zu»), desto höher dürfte wohl die Wahrscheinlichkeit der unmittelbaren Internalisation und Befolgung einer mit Wesentlichkeit begründeten Wertzuschreibung sein.

Problematisch dürften Wesentlichkeitsargumente denn auch vor allem dann sein, wenn Eltern diese häufig, im Sinne eines festen Bestandteils ihres Motivierungsstils, verwenden: Falls die Kinder die entsprechenden Wertzuschreibungen bereits teilen, sie im Sinne integrierter und identifizierter Regulation (vgl. Deci \& Ryan, 1985; Ryan \& Deci, 2002, 2016) also als übereinstimmend mit ihren eigenen Selbstdefinitionen und selbstbezogenen Zielen wahrnehmen (vgl. Abschnitt 5.5.1.2), dürften sie das häufige elterliche Anmahnen lediglich als lästig oder allenfalls als Zeichen fehlenden Vertrauens interpretieren («das weiß ich längst»). Fällt die wahrgenommene Diskrepanz zwischen den wiederkehrend vermittelten elterlichen Wesentlichkeitsbotschaften und den eigenen task values dahingegen groß aus, dürfte das Kind die elterliche Regulation nicht als strukturgebende «guidance» (Skinner, E. A. et al., 2009, S. 65), sondern als, dirigierend, einschränkend und aufdringlich (vgl. Abschnitt 2.2.2.4, insb. Tabelle 2.1) im Sinne intrusiver Kontrolle (coersion) (vgl. Grolnick \& Ryan, 1989; Skinner, E. A. et al., 2009) wahrnehmen und allenfalls ein vordergründiges, wenig nachhaltiges Zustimmen und Befolgen oder aber ein Auflehnen gegen die als anmaßend und autonomiegefährdend empfundenen, mit den eigenen Selbstbildern nicht übereinstimmenden Aussagen an den Tag legen. Der wahrgenommene Druck dürfte für die Kinder auch hier umso größer sein, je geringer ihre Kontrollüberzeugungen hinsichtlich der als wesentlich deklarierten schulischen Aktivitäten und Ziele ausfallen.

Welches Schlussfazit lässt sich nach der Erörterung der drei Dimensionen Valenz, Lokus und Aufgabenwert bezüglich der Effektivität der acht Begründungstypen für elterliche Bedeutsamkeitszuschreibungen ziehen (vgl. Abbildung 5.4)?

Wie bereits im Zwischenfazit festgehalten wurde, kann Eltern empfohlen werden, ihre Bedeutsamkeitszuschreibungen generell möglichst mit Argumenten zu begründen, die positiv-valente und internale Aspekte miteinander kombinieren (Begründungstypen $\mathrm{Oi}+$ und $\mathrm{Ei}+\mathrm{z}$. B. «weil du es dann kannst» bzw. «weil du es ja eigentlich schon kannst»). Mit solchen Begründungen heben sie die anziehenden Aspekte (besseres Verstehen, größeres Wissen, Spaß, bereits bestehende Kompetenzen) hervor, welche mit der angesprochenen Aktivität verbunden sind, sie lösen dadurch angenehme Emotionen (Hoffnung, Zuversicht) aus, die zusammen mit einer optimistischen Kontrolleinschätzung (bzw. einer entsprechenden Kontrollregulation durch die Eltern) zu günstigen motivationalen und behavioralen Folgen führen. Durch Betonung internaler Aspekte haben die Eltern sodann 
die Möglichkeit, einen individuellen Beurteilungsmaßstab einzusetzen und auf eine Lernzielorientierung ihres Kindes hinzuwirken («weil du dadurch immer besser wirst) (vgl. Abschnitt 4.2.1.2). Wie oben dargestellt, dürften ZweckmäBigkeitsargumente vom Kind als Ratschläge aufgefasst werden und signalisieren deshalb grundsätzlich Verhandelbarkeit und Entscheidungsfreiheit. Wesentlichkeitsargumente beinhalten dahingegen selbstbezogene Botschaften und dürften vom Kind deshalb kritischer hinsichtlich ihrer Glaubwürdigkeit und Realitätsnähe begutachtet werden. In diesem Sinne sollten Eltern den an sich produktiven Begründungstyp Ei+(«weil du ja eigentlich so bist», «weil du eigentlich das Potential hast») als Motivierungsstrategie gezielt einsetzen, wenn sie sich sicher sind, dass das Kind die Aussage wenigstens im Ansatz teilt. Der Begründungstyp $\mathrm{Oi}+($ (weil du es so lernst», «weil du es dann kannst», «weil du dich dann gut fühlen wirst») scheint dahingegen eine Motivationsstrategie zu sein, die breit und häufig eingesetzt werden kann und sollte.

Mit negativ-valenten internalen Argumenten (Begründungstypen $\mathrm{Oi}-$ und Ei-: z. B. «weil du es sonst nicht kannst» bzw. «weil du nicht jemand bist, der das kann») heben Eltern gegenüber dem Kind dessen persönliche Grenzen und Kosten hervor, die sich ihm bezüglich seiner Kompetenzentwicklung, seinem emotionalen Befinden oder bezüglich seines Entwicklungspotentials stellen. Besonders der Begründungstyp negative internale Wesentlichkeit (Ei-) sollte von den Eltern so wenig wie möglich eingesetzt werden, insofern als sie dabei mit internal unkontrollierbaren Aspekten argumentieren (Es ist bedeutsam, dass du X machst, weil du mit deinen Persönlichkeitsmerkmalen keine andere Wahl hast). Die signalisierte Zwangslage dürfte beim Kind je nach eigener Wert- und Kontrolleinschätzung, vor allem bei einem häufigen Einsatz dieser Strategie, zu Frustration und Hilflosigkeit führen oder aber - beim Eindruck, die Eltern würden seine Kompetenzen verkennen - Ärger und Wut auslösen, was in Streit münden und eine Ablehnung elterlicher Wertregulationen nach sich ziehen dürfte (vgl. Abschnitt 5.7). Beim Einsatz des Begründungstyps negative internale ZweckmäBigkeit (Oi+) dürften Eltern nur dann mit ihrer Warnung zum Kind durchdringen und wie intendiert Angst und Druck auslösen, wenn das Kind dem genannten Zweck («weil du es sonst nicht kannst») selbst auch eine hohe Bedeutung beimisst. Da negative internale Aspekte angesprochen werden, die das Kind nach außen bis zu einem gewissen Grad verbergen kann, dürfte es andernfalls mit Gleichgültigkeit reagieren («na ja, dann kann ich es halt noch nicht so gut»). Beide Argumentationsstrategien, so ist den Eltern in Anbetracht der genannten Problematiken zu raten, sollten so sporadisch wie möglich eingesetzt werden. 
Auch Negativ-valente externale Argumente (Begründungstypen $\mathrm{Oe}-$ und Ee-: z. B. «weil du sonst eine schlechte Note haben wirst» bzw. «weil du das als Schülerin keinesfalls darfst»), so lässt sich den Eltern ferner empfehlen, sollten sie in ihren Wertregulationen grundsätzlich so selten und situationsangemessen wie möglich verwenden, da sie negative Emotionen, im Zusammenspiel mit geringen Kontrolleinschätzungen namentlich Angst, auslösen. Es kann vermutet werden, dass die Elternteile der vorliegenden Studie vor allem den Begründungstyp Oegehäuft eingesetzt haben: Darum bemüht, das Kind zu motivieren und zu beraten, damit es mit Blick auf den Übertrittsentscheid eine möglichst günstige Leistungsentwicklung an den Tag legt, dürften sich etliche mit dem Umstand konfrontiert gesehen haben, dass sich dessen Lern- und Leistungshandeln ebenso wenig wie die Noten plötzlich verbesserten. Dies dürfte sie dazu veranlasst haben, Warnungen auszusprechen: «Es ist bedeutsam, dass du X tust, weil du sonst den Übertritt nicht schaffst». Auch wenn daraus kurzzeitig eine erhöhte Aufmerksamkeit und Leistungssteigerungen resultieren mögen, so dürften sich bei einem gehäuften Einsatz solcher Wertvermittlungsstrategien, die letztlich darauf beruhen, dass das Kind den hohen Wert des Zwecks teilt («Ich will in die Abteilung A») oder aber die Reaktionen des sozialen Umfelds bei einem Misserfolg fürchtet, Druck und Ängste einstellen, die sich langfristig negativ auf das Wohlbefinden, das Interesse und die vertiefte Beschäftigung mit den angesprochenen Aktivitäten auswirken.

Bedeutungszuschreibungen, die positiv valente und externale Aspekte kombinieren (Begründungstypen Oe+ und Ee+: z. B. «weil du so eine gute Note haben wirst» bzw. «weil du das als Schülerin musst»), dürften vermutlich von den allermeisten Eltern im Kontext der Schule am häufigsten eingesetzt werden. Veranlasst durch die von der Schule gesetzten Ereignisse (Hausaufgaben, Prüfungen, Zeugnisse, der Übertritt etc.) erteilen sie Ratschläge (Oe+) und machen Aussagen über an Rollen geknüpfte Handlungsmaximen (Ee+). Sofern das Kind selbst über hohe diesbezügliche Kontrollüberzeugungen verfügt und sich Erfolge und soziale Anerkennung einstellen, dürften sich daraus keine Probleme ergeben, vielmehr ist mit einer Interessenbildung und Übernahme der Bedeutsamkeitszuschreibungen zu rechnen (vgl. Abschnitt 5.5.1). Obwohl Eltern beim Einsatz dieser beiden Argumentationsstrategien nicht wie bei den negativen Begründungstypen explizit auf bedrohliche Aspekte hinweisen, dürfte der Bezug auf Erwartungen des Umfelds und Ereignisse, die den sozialen Vergleich zulassen, vom Kind vor allem dann aber dennoch als druckvoll und angsterzeugend wahrgenommen werden, wenn es die Wertzuschreibung der Eltern teilt, selber aber über geringe Kontrollüberzeugungen verfügt bzw. entsprechendes evaluatives Feedback von den Eltern erhält (vgl. Abschnitt 5.4.1). 
In der folgenden empirischen Untersuchung wird sich weisen, inwiefern die Vermutung zutrifft, dass gerade in der unsicheren Übertrittssituation, in der die meisten der 20 Elternteile in Anbetracht ihrer Aspirationen und der eher mäßigen Leistungen ihrer Kinder selber unter erhöhtem Druck standen (vgl. Abschnitt 3.3) einen Stil verbaler Wertregulation pflegten, der auf einem dieser beiden letzteren Begründungstypen basierte. Trifft ebenso die Annahme zu, dass das generelle evaluative Feedback der Elternteile - ihr Stil verbaler Kontrollregulation - angesichts der bisherigen Leistungssituation ihrer Kinder eher moderat negative Ausprägungen aufweist («du hast es eher nicht im Griff»), so dürfte im Hinblick auf die affektiv-motivationale Lage der Kinder umso entscheidender gewesen sein, in welchem Maß der kommunikative Modus, in dem Elternteile ihre Appelle vermittelt haben, von Dialogizität, Adaptivität und Wärme geprägt war.

\subsection{Die Bedeutung des Kommunikationsmodus und der Beziehungsqualität für die Internalisierung von kontroll- und wertbezogenen Botschaften durch das Kind}

Abschließend soll dem Umstand nochmals eingehender Beachtung geschenkt werden, dass verbale Motivierungshandlungen der Eltern immer Appelle an das Kind sind, seine kontroll- und wertbezogenen Gedanken bezüglich bestimmter schulischer Aspekte (Aktivitäten und Ziele) in der einen oder anderen Art zu verändern. Diese spezifischen Aspekte, die die Eltern gemäß ihren grundlegenden soziokulturell geprägten Werten (vgl. Abschnitt 4.1), ihren allgemeinen bildungsbezogenen Überzeugungen (vgl. Abschnitt 4.2.1.2), kindspezifischen Aspirationen und Erwartungen (vgl. Abschnitt 2.2.2.4) sowie Wahrnehmungen des betreffenden Bildungssystems und seiner Akteure (vgl. Abschnitt 2.2) selber als bedeutsam erkennen (vgl. Abschnitt 2.1, Abbildung 2.1), werden vor dem Kind nicht nur als relevant deklariert ( $\mathrm{X}$ ist bedeutsam», kategoriale Wertung), sondern auch hinsichtlich des Grads ihrer Relevanz («weil nützlich» oder «weil wesentlich», dimensionale Wertung) gekennzeichnet. Ebenso nehmen sie vor dem Kind (zusätzlich) ein Assessment dessen vor, ob («Du hast X im Griff», kategoriale Beurteilung) und in welchem Maß (weil mehr oder weniger internal, stabil und willentlich steuerbar, dimensionale Beurteilung) es bezüglich dieser Aspekte von gelingenden Ergebnissen ausgehen kann.

Vor allem bei denjenigen schulbezogenen Aspekten, denen die Eltern selber große Wichtigkeit (attainment value) beimessen, dürften sie darauf bedacht sein, dass das Kind ihre diesbezüglichen wert- und kontrollbezogenen Botschaften 
möglichst auf Anhieb wahrnimmt, versteht, in ihr Überzeugungssystem einbaut und entsprechend handelt. Wie in den vorangegangenen Kapiteln erörtert, sollten die Eltern aber wegen der engen Assoziationen zwischen Wert- und Kontrollüberzeugungen (vgl. Abschnitt 5.5.1.4) immer wachen Auges für kompetenzbezogene Äußerungen des Kindes (in Form sprachlicher und affektiver Expressionen) sein, wenn sie wertbezogene Regulationen vornehmen und umgekehrt.

So ist bei evaluativen Feedbacks nicht nur darauf zu achten, dass diese vom Kind als sachlich angemessen, argumentativ plausibel und glaubwürdig eingeschätzt werden (vgl. Schunk et al., 2014, S. 118), sondern seinem Bedürfnis nach Schutz des eigenen «sense of competency, respect, and self-acceptance» (Covington, 1992, S. 74) nachkommen (vgl. Abschnitt 5.5.1.4). Ferner sollten Eltern dabei kontrollbezogene Begründungen verwenden (vgl. Abschnitt 5.4.1), die Zuversicht und Hoffnung erzeugen und das Kind auf einen adaptiven AttributionsEmotions-Motivations-Performanz-Pfad (vgl. Perry \& Hamm, 2017) bringen (vgl. Abschnitt 5.3.3.2). Bandura (1997, S. 104) gibt in dieser Hinsicht zu bedenken, dass es generell einfacher sei, mit destruktiven in geringschätzender Weise formulierten und/oder inhaltlich einseitig auf die Defizite abhebenden Rückmeldungen Selbstwirksamkeitsüberzeugungen zu untergraben, als diese mit konstruktivem Feedback allein nachhaltig zu heben:

Devaluative Feedback not only creates social estrangement but undermines people's belief in themselves. Given the same level of performance, disparaging criticism lowers perceived efficacy and aspirations, whereas constructive criticism sustains aspirations and upholds or even bolsters a sense of personal efficacy [...]. (Bandura, 1997, S. 103-104)

Gleichermaßen wurde oben deutlich, dass Eltern bei der Formulierung ihrer Wertzuschreibungen darauf achten müssen, dass diese den Kontrollüberzeugungen der Kinder entsprechen. So ergaben sich in den Interventionsstudien von Canning und Harackiewicz (2015) deutliche Hinweise darauf, dass selbst Bedeutsamkeitszuschreibungen mit Zweckmäßigkeitsargumenten - welche grundsätzlich den Charakter von Ratschlägen aufweisen, eine moderate Wichtigkeit signalisieren und dem Bedürfnis der Rezipient*innen nach Selbstbestimmung bzw. Entscheidungsfreiheit entgegenkommen - zu Angst und Handlungsblockaden führen, wenn das Individuum bezüglich der konkret genannten Aktivität ( $\ll \mathrm{X}$ ist bedeutsam») oder des spezifisch genannten Handlungszwecks («damit du Y erreichst») lediglich ein geringes Vertrauen in seine eigenen Fähigkeiten aufweist. Die Experimente zeigten ferner auf, wie wichtig es im Hinblick auf die Internalisierung und Handlungsausführung ist, dass die Lernenden die Möglichkeit haben, ihre 
eigenen Überlegungen bezüglich des persönlichen Nutzens anzustellen und zu verbalisieren. Die für die Rezeption und Internalisation entscheidende Passung mit den Werten und dem inhaltlichen (Vor-)Wissen des Kindes, so lässt sich folgern, sollte über ein möglichst dialogisches Vorgehen sichergestellt werden (vgl. Abschnitt 5.6.2.3). Dies dürfte insbesondere bei Bedeutsamkeitszuschreibungen gelten, die der angesprochenen Aktivität bzw. dem angesprochenen Handlungsziel Wesentlichkeit zuschreiben und damit eine Notwendigkeit der Befolgung implizieren, die als Zwang interpretiert werden kann. Teilt das Kind den signalisierten hohen Wert (attainment value) nicht wenigstens in Ansätzen und vertraut es vor allem seinen diesbezüglichen Fähigkeiten nicht, so dürfte es Angst wegen der angedeuteten hohen Bedeutung noch intensiver erleben als bei Argumenten, die den utility value hervorheben (vgl. Frenzel \& Stephens, 2017, S. 38-41).

Aus den in Abschnitt 5.5.1.3 berichteten Befunden der Interventionsstudien von Simons et al. $(2003,2004)$ lässt sich ferner schließen, dass es ratsam sein dürfte, dass Eltern die von ihnen wohl häufig eingesetzten, aber mit Zwang und Druck assoziierten externalen Wesentlichkeitsargumente («weil du musst», «weil du nicht darfst») grundsätzlich so sparsam wie nötig und so situationsangemessen wie möglich einsetzen (z. B. bei Gefahren oder sich abzeichnenden schwerwiegenden Fehlern) und an deren Stelle möglichst mit positiver Zweckmäßigkeit einer Aktivität argumentieren sollten. Das Erkennen eines persönlichen utility values geht mit erhöhtem Engagement und verbesserten Leistungsergebnissen einher (Johnson \& Sinatra, 2013) (vgl. Abschnitt 5.5.1.3). Eltern können darauf hoffen, dass sich ihr Kind mit der Beschäftigung zunehmend stärker für die Aktivität interessiert und sich mit dem Gelingen nicht nur steigende Kontrollüberzeugungen ergeben, sondern auch eine steigende Identifizierung mit der Aktivität einsetzt und somit im Sinne der Organismic Integration Theory (vgl. Deci \& Ryan, 1985; Ryan \& Deci, 2002, 2016) (vgl. Abschnitt 5.5.1.2) die persönliche Wichtigkeit derselben steigt.

Eltern, so wird deutlich, «[...] walk a very fine line between being supportive and being overcontrolling» (Jacobs \& Eccles, 2000, S. 430). Um mit ihren Wert- und Kontrollzuschreibungen erfolgreich zu sein, dürfte nicht nur entscheidend sein, wie die Eltern diese argumentativ gestalten - situationsangemessen, verständlich und glaubwürdig -, sondern auch in welchem kommunikativen Modus sie ihre Forderungen nach Internalisierung und Befolgung (in den Frames mit dem einleitenden «Merke dir!» ausgedrückt, vgl. Abschnitte 5.4.1 und 5.6.1) erheben (vgl. Grusec, 2011, S. 252). Gerade bei potentiell selbstwertgefährdenden und autonomieeinschränkenden Aussagen dürfte nicht nur bedeutsam sein, wie die veränderten Wert- und Kontrolleinschätzungen situativ vom Kind 
eingefordert werden, sondern wer die Person ist und in welchem «emotional climate» sie generell ihre Appelle an das Kind vornimmt (Darling \& Steinberg, 1993, S. 493).

Appelle sind in der Sprechaktklassifikation von Searle (1976, S. 11) sog. Direktiva (directives), also Sprechhandlungen, mit denen Forderungen an das Gegenüber gerichtet werden bzw. mit denen dieses zu einer Handlung bewegt werden soll:

[Directives] may be very modest 'attempts' as when I invite you to do it or suggest that you do it, or they may be very fierce attempts as when I insist that you do it. [...] The direction of fit is world-to-words and the sincerity condition is want (or wish or desire).The propositional content is always that the hearer $\mathrm{H}$ does some future action A. Verbs denoting members of this class are ask, order, command, request, beg, plead, pray, entreat, and also invite, permit, and advise. (Searle, 1976, S. 11)

Die Verben, die Searle hier als kennzeichnend für direktive Sprechakte aufzählt - insofern als sie alle sog. Welt-an-Wort-Anpassungen signalisieren -, illustrieren die Bandbreite, mit der Forderungen formuliert werden können: Sie reicht von «befehlen» bis zu «bitten», wobei auch eine explizite Bitte je nach situativen Bedingungen, der Mimik, Gestik und Proxemik der sprechenden Person sowie weiteren sprachlichen Markern ${ }^{30}$ von der rezipierenden Person ebenfalls als eine bindende Aufforderung interpretiert werden kann. Kinder werden u. a. darauf achten, welche Emphase die Eltern in den Appell legen, um zu prüfen, inwiefern deren Aufforderung, ein verändertes Verhalten zu zeigen, zum Nennwert zu nehmen ist. Mit Blick auf Searles Worte im obigen Zitat stellt sich für das Kind (und die Forschenden), die Frage, ob es sich bei den Überzeugungsversuchen der Eltern nur um «modest attempts» oder um ernstzunehmende «fierce attempts» handelt.

Das Kind wird ferner registrieren, ob die Eltern auch bei seiner expliziten Ablehnung der Botschaft auf dieser insistieren oder ob sie diese relativieren, sobald ihnen sein Widerspruch und sein Trotz entgegenschlägt («Ich finde zwar, dass du... solltest - aber du musst es selber wissen.»). Letzteres dürfte bezogen auf die konkrete Situation, vor allem aber situationsübergreifend, wenn dies gehäuft dem Kommunikationsverhalten der Eltern bei Wert- und Kontrollzuschreibungen entspricht, das strukturgebende Potential (vgl. Abschnitt 2.2.2.4, insb. Tabelle 2.1) dieser Unterstützungsformen untergraben. Mangelt es den verbalen

\footnotetext{
${ }^{30}$ Solche sprachlichen Marker signalisieren u. a. Gefühle, die Dringlichkeit und das Verhältnis zwischen den Kommunikationspartnern im Sinne von Nähe/Distanz und sozialen Statusunterschieden (Sozialdeiktika) (vgl. Linke et al., 2004, S. 219-232).
} 
Regulationen an Konsistenz - widersprechen sich die Botschaften über die Zeit, widersprechen sich die Botschaften und die Prägnanz, mit der sie vertreten werden und/oder widersprechen sich die Botschaften und das eigene Handeln der Eltern (vgl. Farkas \& Grolnick, 2010, S. 268; Pomerantz, Kim \& Cheung, 2012, S. 423-424) - dürfte das Kind entsprechende Appelle als unstet, willkürlich und unglaubwürdig im Sinne von chaos (vgl. Skinner, E. A. et al., 2009, S. 221) (vgl. Abschnitt 2.2.2.4, insb. Tabelle 2.1) wahrnehmen. Dies dürfte nicht nur mit negativen Effekten hinsichtlich der Internalisierung und Befolgung der Regulationen einhergehen, sondern auch negative Auswirkungen auf die Autorität der Eltern haben (Apel, 2002, S. 117-120; Reichenbach, 2011, S. 26-30; Schäfer \& Thompson, 2009; Sofsky \& Paris, 1991). Ein Kennzeichen dafür, dass die Kinder ihre Eltern als valide Normsetzer und -beurteiler anerkennen - «dass wir es [...] mit Autorität zu tun haben» - besteht laut Krüger (1953, S. 54) darin, «dass eine Person irgendwie 'maßgebend' ist - dass sie anderen 'etwas zu sagen hat', während sich die anderen etwas von ihr 'sagen lassen' [...]».

Eltern dürften namentlich dann Aufmerksamkeit wecken und überzeugend wirken, wenn sie kommunikativ jene «ruhige Festigkeit» oder «Nicht-verletzende[] Geltendmachung des eigenen Standpunkts» an den Tag legen, die Aebli (1997, S. 273) als «Assertivität» bezeichnet: Wenn sie aktiv das Kind ansprechen und Stellung beziehen, sich nicht gleich seinem Widerspruch bzw. Nicht-Befolgen beugen und dabei ihre Gefühle in kontrollierter, das Gesicht des Kindes wahrenden Weise zum Ausdruck bringen ${ }^{31}$. Eine solchermaßen an den Tag gelegte Verbindlichkeit und Verantwortlichkeit, die insofern autoritätsförderlich und vertrauensbildend wirkt, als sich die Eltern als Expertinnen und Experten für den schulischen Realitätsbereich präsentieren (vgl. Sofsky \& Paris, 1991, S. 27), bedingt allerdings, dass diese ihre Botschaften auf Nachfrage des Kindes mittels Anschlussargumenten u. a. in Form von Eigenerfahrungen zu plausibilisieren vermögen (vgl. Kapitel 4). Damit die Strukturgebung, die die Eltern mit ihrem festen Vertreten ihrer Überzeugungen sicherstellen, in den Augen des Kindes nicht als Zwang (coersion) bzw. als autokratisches Verhalten (vgl. Abschnitt 2.2.2.4, insb. Tabelle 2.1) erscheint, dürfte es denn auch wichtig sein, dass die Kontroll-

\footnotetext{
${ }^{31}$ Aebli (1997) spricht hierbei von aktiver Assertivität - «[...] es wagen, den anderen anzugehen und seine Aufmerksamkeit in Anspruch zu nehmen» (S. 275) -, von reaktiver Assertivität - «[...] fähig sein, Zumutungen und Forderungen, deren Befriedigung [einen] selbst schädigen oder dem anderen nicht wirklich dienen würde, abzulehnen, oder ihnen zumindest nur in Grenzen nachzukommen» (S. 274) - sowie von expressiver Assertivität (S. 275-276), der Fähigkeit, die eigenen Gefühle und Standpunkte unter gleichzeitiger Berücksichtigung der Bedürfnisse und Standpunkte des andern kontrolliert als sog. «Ich-Botschaften» (vgl. Gordon, 1972, S. 104-135) auszudrücken.
} 
und Wertregulationen nicht monologisch bleiben, Widerspruch und Rückfragen des Kindes nicht ins Leere laufen oder auf stures Beharren treffen (vgl. Grusec, 2012).

Gerade in jenen heiklen schulbezogenen Situationen, in denen das Kind Misserfolge zu verkraften hat, die Eltern unrealistisch hohe Erfolgserwartungen des Kindes zu dämpfen versuchen oder in denen es von Ängsten geplagt wird wenn also unter Berücksichtigung der psychologischen Bedürfnisse des Kindes ein realitätsnahes, Leistungen und Leistungsanforderungen sowie eigene Erwartungen benennendes verbales Handeln gefragt ist - dürfte ein hoher Grad an Dialogizität notwendig sein. Kinder sollten regelmäßig, besonders aber in solch delikaten Situationen, erleben, dass sich ihre Eltern bemühen, ihre Perspektiven einzunehmen, ihnen die Möglichkeit zu geben, die Situationen mit Bezug auf ihre persönlichen Überlegungen darzulegen, ihnen entsprechend angepasstere, plausiblere Erklärungen, Erläuterungen und Beispiele anzubieten und sich zurückzunehmen, wenn sie ihr Verstehen oder ein genügendes Maß an Internalisierung erkennen lassen. Wenn Eltern gegenüber ihrem Kind eine Haltung erkennen lassen, die die eigenen Kontroll- und Wertzuschreibungen im Sinne Vygotskijs (2002) und der Literatur zu Tutoring, Mentoring und Coaching (z. B. Chi, Siler, Jeong, Yamauchi \& Hausmann, 2001; Collins, Brown \& Newman, 1989; Graesser, Conley \& Olney, 2012; Staub, 2004; Tharp \& Gallimore, 1988) als psychologische oder semantische Werkzeuge versteht, die sich das Kind erst zu eigen machen und hinsichtlich ihrer Brauchbarkeit in seiner schulbezogenen Realität erproben muss, wenn sie ein Verständnis für entwicklungsbedingte Positionsbezüge des Kindes im Übergang zum Jugendalter erkennen lassen (vgl. Jacobs \& Eccles, 2000, S. 415) und unter Beweis stellen, dass ihre eigenen Einschätzungen und Überzeugungen in den Gesprächen mit dem Kind wandeln (vgl. Simpkins \& Fredricks, 2015, S. 926) -, dürften sie nicht nur für die akkurate Wahrnehmung der Botschaften, sondern ebenso die Wahrscheinlichkeit erhöht haben, dass diese vom Kind auch akzeptiert werden (vgl. Grusec, 2011, S. 252).

Damit «Akzeptanz» allerdings nicht nur situative Zustimmung und Befolgung des elterlichen Appells, sondern ein «inner indorsement» der ausgedrückten Botschaft bedeutet, reichen ein von hoher Assertivität und hoher Dialogizität geprägter Kommunikationsstil, der das Bedürfnis noch Kompetenz- und Autonomieerleben befriedigt, allein nicht aus: «Children will be most likely to internalize regulations when relatedness needs are met, meaning when they have a positive relationship with an involved, supportive parent» (Grolnick, 2003, S. 56).

Damit Kinder es wagen, ihre eigenen Sichtweisen und Befürchtungen frei zum Ausdruck zu bringen, damit sie auf die Botschaften und Argumente fokussiert bleiben und sich nicht stattdessen stärker mit dem Selbstwertschutz und Gedanken 
zur Beziehungsqualität beschäftigen (vgl. Wood et al., 1976, S. 98), ist es notwendig, dass die Kinder sich in der einzelnen Regulationsepisode, letztlich aber stabil über die wert- und kontrollbezogenen Gespräche hinweg, trotz seines Widerspruchs und Nachfragens, trotz seiner augenscheinlich geringen Einsicht und mitunter zum Ausdruck gebrachten negativen Affekte stets als Persönlichkeiten anerkannt und wertgeschätzt fühlen. Erleben die Kinder ihre Eltern (und den Rest der Familie) als sichere «refuling base» (Bodenmann, 2016, S. 88), die gerade auch in konfliktbehafteten Situationen im Sinne der Qualitätsdimension warmth (vgl. Abschnitt 2.2.2.4, insb. Tabelle 2.1) keinen Zweifel an ihrer Zuneigung und Achtung aufkommen lassen, indem sie auf herabsetzende und feindselige Äußerungen verzichten, von Kommunikationsverweigerung und «Missachtung» sowie von «Kälte» und «Härte» (Tausch \& Tausch, 1998, S. 102) absehen und gegebenenfalls die Bereitschaft zeigen, sich freimütig $\mathrm{zu}$ entschuldigen, sollten sie situativ Verhaltensformen zeigen, die Skinner und Kolleg*innen (2009, S. 186) der rejection zuordnen (vgl. Abschnitt 2.2.2.4, insb. Tabelle 2.1), so schaffen sie jenen unterstützenden häuslichen Kontext, der die Internalisierung elterlicher schulbezogener Werte und Erwartungen beim Kind befördert (u. a. Grolnick \& Ryan, 1989; Grolnick et al., 1991; Simpkins, Weiss, McCartney, Kreider \& Dearing, 2006; zsf. Wigfield, Eccles, et al., 2015, S. 25).

\subsection{Elterliche Wert- und Kontrollregulationen im Kontext eines unklaren Übertrittsentscheids: Fragestellungen und Aufbau der empirischen Untersuchung}

Die Hauptziele der vorliegenden Studie bestehen einerseits in einer möglichst facettenreichen Deskription der Praktiken, mit denen die 20 Elternteile in den acht Monaten vor dem Übertrittsentscheid versucht haben, ihre Tochter oder ihren Sohn verbal-appellativ für schulische Aktivitäten und Ziele zu motivieren. Andererseits sollen die jeweiligen elterlichen Motivierungsstile auf der Basis von Fallbeispielen sowie mit Blick auf die in den vergangenen Kapiteln erörterten Befunde und Postulate hinsichtlich ihrer Chancen und Risiken für ein in motivational-affektiver Hinsicht produktives Bewältigen der zugespitzten Leistungssituation beurteilt werden.

Wie in den vergangenen Kapiteln erörtert, ist es unbestritten, dass Eltern während der Kindheit und bis weit ins Jugendalter hinein mit ihren vorgelebten sowie verbal und nonverbal zum Ausdruck gebrachten schulbezogenen Werten, Aspirationen und Leistungserwartungen einen starken Einfluss auf die Entwicklung von wert- und kontrollbezogenen Einschätzungen und Überzeugungen bei ihrem 
Sohn oder ihrer Tochter ausüben (vgl. Abschnitt 4). Immer wieder zeigt sich in Einklang mit den Postulaten der Selbstbestimmungstheorie, aber auch der Erziehungsstilforschung (vgl. Abschnitt 2.2.2.4), dass für die Entwicklung dieser für die Motivation zentralen schulbezogenen Überzeugungen ein autonomieförderlicher, strukturgebender sowie emotional zugewandter Unterstützungsstil aufseiten der Eltern notwendig ist. Angesichts der bisher fast ausschließlich auf quantitativen Designs beruhenden Untersuchungen ist allerdings wenig dazu bekannt, wie Eltern konkret vorgehen, wenn sie ihr Kind zu motivieren versuchen. Unklar ist insbesondere, wie sie ihre verbalen Praktiken gestalten, mit denen sie ihr Kind zu veränderten Einschätzungen schulischer Aktivitäten und Ziele bewegen möchten. Je älter die Kinder werden und je dringlicher den Eltern eine diesbezüglich angepasste Motivation erscheint, so kann angenommen werden, desto stärker setzen sie darauf, ihre eigenen schulbezogenen Wahrnehmungen und Überzeugungen dem Kind sprachlich explizit zu vermitteln. Gerade ein unsicherer Übertrittsentscheid dürfte denn auch bei vielen Eltern zu einem erhöhten Einsatz dieser verbal-appellativen Unterstützungsformen führen (vgl. Abschnitt 4.3).

Verbales Motivieren, so wurde in den vorangegangenen Abschnitten des Kapitels 5 deutlich, besteht aus der Perspektive von erwartungswert-theoretischen Konzeptionen der Lern- und Leistungsmotivation im Kern in sprachlichen Interventionen der Eltern, die darauf abzielen, die Wert- und die Kontrollzuschreibungen des Kindes so zu verändern, dass sie ihren eigenen Zielen und Gütemaßstäben bzw. denjenigen der Klassenlehrkräfte entsprechen (vgl. Abschnitt 2.2), deren Diagnose und Empfehlung im Übertrittsverfahren der Volksschule des Kantons Zürich ein großes Gewicht zukommt (vgl. Abschnitt 3.3).

In den Aussagen der 20 Elternteile, die sie im Rahmen eines ausführlichen, auf die schul- und kindbezogenen Ereignisse der letzten Monate zurückblickenden, kurz nach dem Übertrittsentscheid durchgeführten Interviews gemacht haben (vgl. Abbildung 6.1), wurden im Analyseschritt (A) - der sog. Basiscodierung 194 wertbezogene und 104 kontrollbezogene Handlungsepisoden isoliert, in denen die Elternteile schildern, wie sie im Gespräch mit ihrem Kind Bedeutsamkeitszuschreibungen und evaluative Feedbacks bezüglich bestimmter schulischer Aspekte vorgenommen haben (vgl. Abschnitt 6.4.3).

Nach einer ausführlichen Darstellung des Untersuchungsdesigns werden in den folgenden Kapiteln die Ergebnisse der Analysen vorgestellt, die an den insgesamt 298 Handlungsepisoden durchgeführt wurden. Die Episoden wurden in mehreren Schritten, die sich hinsichtlich des Grades der Textnähe bzw. des Grades an Deutungs- und Interpretationsleistungen bei der Analyse unterscheiden (vgl. Abschnitt 6.4.2), mit Hilfe der beiden in Abschnitt 5.4.1 und Abschnitt 5.6.1 vorgestellten Frames zuerst fallübergreifend themenorientiert 
bezüglich der Häufigkeit bestimmter Gestaltungsmerkmale von Wert- und Kontrollregulationen untersucht und dann darauf aufbauend fallorientiert hinsichtlich des Auftretens bestimmter Merkmalsmuster bei den jeweiligen Elternteilen ausgewertet. Im letzten Analyseschritt (vgl. Abschnitt 6.4.4) wurden die Elternteile mittels statistischer Algorithmen aufgrund von Ähnlichkeiten und Unterschieden in den Merkmalsmustern ihres sprachlichen Handelns zu Clustern zusammengefasst, die einen bestimmten Stil verbaler Motivierung während der unsicheren Übertrittsphase repräsentieren.

Geordnet nach den Analyseschritten (B) bis (D) werden im Folgenden die Haupt- und Unterfragen der empirischen Studie dargestellt.

(B) Fallübergreifende, auf die Häufigkeit von Gestaltungsmerkmalen der Wertund Kontrollregulationen ausgerichtete, mittels der niedrig-inferenten inhaltlich strukturierenden qualitativen Inhaltsanalyse (vgl. Kuckartz, 2018, S. 77-98) ausgewertete Fragestellungen:

1. Wie gestalten die 20 Elternteile im Kontext eines unklaren Übertrittsentscheids in die Sekundarstufe I ihre verbal-appellativen Wertregulationen bzw. Bedeutsamkeitszuschreibungen?

1.1. $\mathrm{Zu}$ welchen konkreten lern- und leistungsthematischen Zielen berichten die Elternteile von eigenen wertbezogenen verbal-appellativen Regulationen gegenüber ihren Kindern?

1.2. Wie verteilen sich die in den 194 wertbezogenen Episoden fokussierten Lern- und Leistungsziele, wenn sie nach Zielbereichen geordnet werden, die aus den vier Kompetenzklassen nach Erpenbeck und von Rosenstiel (2003) (vgl. Abschnitt 2.2.2.1) sowie der Kategorie «Leistungsergebnis» gebildet wurden?

1.3. Welche konkreten Argumente führen die Elternteile in den 194 wertbezogenen Episoden den Kindern als Begründung der Bedeutsamkeit schulischer Lern- und Leistungsziele vor Augen?

1.4. Wie verteilen sich die in den 194 Motivierungsepisoden eingesetzten Argumente, wenn sie nach Begründungstypen geordnet werden, die aus den Dimensionen Valenz (positiv vs. negativ), Aufgabenwert (Wesentlichkeit und Zweckmäßigkeit) sowie Lokus (internal vs. external) gebildet wurden (vgl. Abbildung 5.4)?

1.5. $\mathrm{Zu}$ welchen Begründungstypen greifen die 20 Elternteile in den vorgefundenen Handlungsepisoden am häufigsten, wenn sie ihren Kindern die Bedeutsamkeit einer bestimmten Klasse von Lern- und Leistungszielen $\mathrm{zu}$ vermitteln suchen? 
2. Wie gestalten die 20 Elternteile im Kontext eines unklaren Übertrittsentscheids in die Sekundarstufe I ihre verbal-appellativen Kontrollregulationen bzw. evaluativen Feedbacks?

2.1. $\mathrm{Zu}$ welchen konkreten lern- und leistungsthematischen Zielen berichten die Elternteile von eigenen kontrollbezogenen verbal-appellativen Regulationen gegenüber ihren Kindern?

2.2. Wie verteilen sich die in den 104 kontrollbezogenen Episoden fokussierten Lern- und Leistungsziele, wenn sie nach Zielbereichen geordnet werden, die aus den vier Kompetenzklassen nach Erpenbeck und von Rosenstiel (2003) sowie der Kategorie «Leistungsergebnis» gebildet wurden?

2.3. Welche konkreten prozessualen und/oder strukturellen Merkmale führen die Elternteile in den 104 kontrollbezogenen Episoden den Kindern als Argumente zur Begründung für deren Kontrolle schulischer Lern- und Leistungsziele vor Augen?

2.4. Wie verteilen sich die in den 104 Kontrollepisoden gesamthaft eingesetzten 140 Argumente, wenn sie nach Begründungstypen geordnet werden, die aus den Dimensionen Valenz (positiv vs. negativ), Lokus (internal vs. external), Stabilität (stabil vs. variabel) sowie Willentliche Beeinflussbarkeit (hoch vs. tief) (vgl. Abbildung 5.3) gebildet wurden?

2.5. Bezüglich welcher Lern- und Leistungsziele attestieren die Elternteile ihren Kindern in den vorgefundenen Handlungsepisoden auf der Basis der vor Augen geführten Begründungen eine hohe bzw. eine geringe Kontrolle (vgl. Tabelle 5.2)?

(C) Fallspezifische, auf die Merkmale des individuellen Regulationsstils ausgerichtete, mittels der höher-inferenten evaluativen qualitativen Inhaltsanalyse (vgl. Kuckartz, 2018, S. 98-115) ausgewertete Fragestellungen:

3. Welche Eigenschaften weist der individuelle Stil des verbal-appellativen wertund kontrollbezogenen Handelns der 18 in diese Analysen aufgenommenen Elternteile ${ }^{32}$ während der unsicheren Übertrittszeit auf?

3.1. Welches waren die individuell dominanten Argumentationselemente, mit denen die einzelnen Elternteile die Bedeutsamkeitszuschreibungen ihres Kindes zu regulieren suchten?

\footnotetext{
${ }^{32}$ Wie in Abschnitt 6.4.1.3 näher erläutert wird, wurden die Elternteile S12 und Z21 aufgrund zu weniger Textstellen für die stilbezogenen Analysen der Fragestellungen 3 und 4 ausgeschlossen.
} 
3.2. Welches waren die individuell dominanten Argumentationselemente, mit denen die einzelnen Elternteile die Kontrolleinschätzungen ihres Kindes zu regulieren suchten?

3.3. Welches waren die individuell dominanten kommunikativen Stilelemente, mit denen die einzelnen Elternteile ihr Kind von eigenen Wert- und Kontrollzuschreibungen zu überzeugen suchten?

3.4. In welchem emotionalen Klima fanden die individuellen elterlichen Wert- und Kontrollregulationen statt, gemessen an den selbstberichteten Reaktionen auf Erfolge und Misserfolge des Kindes während der Übertrittszeit?

(D) Gruppenspezifische, auf die Identifikation der zwischen den 18 Elternteilen vorherrschenden Ähnlichkeiten und Unterschiede in ihrem verbalen Motivierungshandeln ausgerichtete, mittels der Hauptkomponentenanalyse, des k-means Clustering sowie Konfigurations- und Zusammenhangsanalysen ausgewertete Fragestellungen:

4. Wie lässt sich das schulbezogene verbale Motivierungshandeln der 18 Elternteile gruppieren und was sind die Charakteristika jedes Motivierungstyps?

4.1. Auf welche latenten «grundlegenden Dimensionen elterlichen verbalen Motivierens bei einem unklaren Übertrittsentscheid» lassen sich die manifesten wert- und kontrollbezogenen sowie kommunikations- und beziehungsbezogenen Stilelemente der 18 Elternteile verdichten?

4.2. Wie gruppieren sich die 18 Elternteile bezüglich dieser «grundlegenden Dimensionen des schulbezogenen verbalen Motivierens bei einem unklaren Übertrittsentscheid»?

4.3. Welches sind die Charakteristika des jeweiligen gruppenspezifischen Motivierungsstils - der ermittelten «Typen verbal-appellativen Motivierens bei einem unsicheren Übertritt»?

4.4. Welche Zusammenhänge zeigen sich zwischen einer Reihe von Eltern-, Kind- und Familienmerkmalen und den gebildeten Typen (vgl. Abschnitt 4.3)?

Eine nähere Erörterung der Fragestellungen erfolgt jeweils an der entsprechenden Stelle im Ergebnisteil. Im abschließenden Kapitel 8 werden die Befunde zusammengefasst und mit Blick auf die eingangs formulierten Zielsetzungen diskutiert: Jeder der ermittelten gruppenspezifischen elterlichen Motivierungsstile soll mit Bezug auf die erörterten Forschungsbefunde und theoretischen Postulate, aber 
auch mit Blick auf einzelne Fallbeispiele hinsichtlich der Potentiale und Gefahren eingeschätzt werden, die mit ihm betreffend motivational-affektiver Aspekte bei den Kindern verbunden sind. Es handelt sich dabei um ein hypothesengenerierendes Vorgehen, wobei das Diskussionskapitel nicht nur mit Schlussfolgerungen für die weitere Forschung, sondern mit ebensolchen für die pädagogische Praxis aufwartet. Im Folgenden wird nun das Design der Untersuchung ausführlich erläutert.

Open Access Dieses Kapitel wird unter der Creative Commons Namensnennung 4.0 International Lizenz (http://creativecommons.org/licenses/by/4.0/deed.de) veröffentlicht, welche die Nutzung, Vervielfältigung, Bearbeitung, Verbreitung und Wiedergabe in jeglichem Medium und Format erlaubt, sofern Sie den/die ursprünglichen Autor(en) und die Quelle ordnungsgemäß nennen, einen Link zur Creative Commons Lizenz beifügen und angeben, ob Änderungen vorgenommen wurden.

Die in diesem Kapitel enthaltenen Bilder und sonstiges Drittmaterial unterliegen ebenfalls der genannten Creative Commons Lizenz, sofern sich aus der Abbildungslegende nichts anderes ergibt. Sofern das betreffende Material nicht unter der genannten Creative Commons Lizenz steht und die betreffende Handlung nicht nach gesetzlichen Vorschriften erlaubt ist, ist für die oben aufgeführten Weiterverwendungen des Materials die Einwilligung des jeweiligen Rechteinhabers einzuholen. 